PBH 224-702.\$5.50

\title{
LABORATORY EVALUATION OF ALCOHOL SAFETY INTERLOCK . SYSTEMS, VOLUME III - INSTRUMENT PERFORMANCE AT HIGH BAL
}

John F. Oates, Jr.

Robert T. McCay

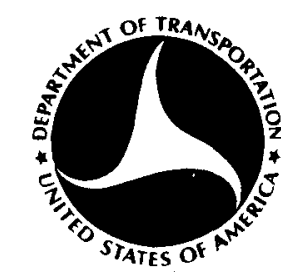

\author{
REPRINT \\ January 1974 \\ FINAL REPORT
}

\begin{abstract}
DOCUMENT IS AVAILABLE TO THE PUBLIC
THROUGH THE NATIONAL TECHNICAL

INFORMATION SERVICE, SPRINGFIELD.

VIRGINIA 22151.
\end{abstract}

\author{
Prepared for \\ DEPARTMENT OF TRANSPORTATION \\ NATIONAL HIGHWAY TRAFFIC SAFETY ADMINISTRATION \\ Research Institute \\ Washington DC 20591
}




\section{NOTICE}

This document is disseminated under the sponsiorship of the Department of Transportation in the interest of information exhange. The United States Government assumes no liability for its contents or use thereof.

\section{NOTICE}

The United States Government does not endorse products or manufacturers. Trade or manufacturers' names appear herein solely because they are considered essential to the object of this report. 
TECHNICAL REPORT STANDARD TITLE PAGE

\begin{tabular}{|c|c|}
\hline $\begin{array}{l}\text { 2. Government Accession No: } \\
\text { PB- } 224702\end{array}$ & 3. Recipient's Catalog No. \\
\hline 4. Titlo and subritle LABORATORY EVALUATION OF ALCOHOL & $\begin{array}{l}\text { 5. Roport Dato } \\
\text { January } 1974\end{array}$ \\
\hline $\begin{array}{l}\text { MENT PERFORMANCE AT HIGH BAL. } \\
\text { MEN }\end{array}$ & 6. Performing Orgonization Code \\
\hline $\begin{array}{l}\text { 7. Author(s) } \\
\text { John F. Oates, Jr., Robert T. McCay }\end{array}$ & $\begin{array}{l}\text { 8. Porforming Organizotion Report No. } \\
\text { DOT-TSC-NHTSA-73-3, I I I }\end{array}$ \\
\hline $\begin{array}{l}\text { 9. Performing Organizotion Nome and Address } \\
\text { Dunlap \& As sociates, Inc.* }\end{array}$ & $\begin{array}{l}\text { 10. Work Unit No. } \\
\text { HS 306/R3407 }\end{array}$ \\
\hline $\begin{array}{ll}\text { Dne Parkland Drive } \\
\text { Darien CT } & 06820\end{array}$ & $\begin{array}{l}\text { 11. Contract or Grant No. } \\
\text { DOT - TSC - } 251\end{array}$ \\
\hline & 13. Type of Report and Period Covered \\
\hline $\begin{array}{l}\text { 12. Sponsoring Ageney Nome and Addross } \\
\text { Department of Transportation } \\
\text { National Hafety }\end{array}$ & $\begin{array}{l}\text { Fina1 Report } \\
8 / 71-10 / 72\end{array}$ \\
\hline $\begin{array}{l}\text { Research Institute } \\
\text { Washington DC } 20591\end{array}$ & 14. Sponsoring Ageney Code \\
\hline $\begin{array}{l}\text { 15. Supplementary Nores * Under Contract to: } \\
\text { Reprint }\end{array}$ & $\begin{array}{l}\text { of Transportation } \\
\text { ion Systems Center } \\
\text { are, Cambridge MA }\end{array}$ \\
\hline
\end{tabular}

This ${ }^{6}$ repstroct contains the results of an experimental and analytical evaluation of instruments and techniques designed to prevent an intoxicated driver from operating his automobile. The prototype "Alcohol Safety. Interlock Systems" tested were developed both by private industry and by the Transportation Systems Center and were all drawn from a class of instruments which detect intoxication by measuring changes in the subjects ability to perform a psychomotor task. The final report consists of the following documents:

Volume I, Summary Report - Contains an overview and summary of a11 of the ASIS evaluation work performed through July 1972 and the results of the evaluation. Volume $I$ is divided between the overview and an extensive appendix.

Volume II, Instrument Screening Experiments - Contains details of the experiments conducted by the Guggenheim Center, Harvard School of Public Health, including experimental procedures, results and some preliminary data analyses.

Volume III, - Instrument Performance at High BAL - Contains the results of the experimental work performed by Dunlap and Associates, Inc., covering the performance of subjects with relatively high blood alcohol levels on selected instruments.

17. Koy Word:
alcohol, intoxication, interlock,
intoxicated performance, habitual
drinker

\section{Distribution Statement}

Document is availagle to the public THROUGH THE NATIONAL TECHNICAL INFORMATION SERVICE, SPRINGFIELO. VIRGINIA 22151. 
This report documents the results of laboratory testing of five prototype Alcohol Safety Interlock Systems as conducted by the staff of Dunlap and Associates, Inc., for the Transportation Systems Center. The period of time covered by this report is March 1972 to August 1972 .

The authors wish to acknowledge their indebtedness to the numerous individuals and organizations who contributed to the successful conduct of this program. The following, in particular, deserve special mention for the key roles they played:

- Mr. Philip W. Davis of the Transportation Systems Center, the Contract Technical Manager, and Mr. Joseph T. Fucigna, Executive Vice President of Dunlap and Associates, Inc., who provided overall guidance, timely suggestions, and constant encouragement;

- Drs. Edward Rem, Howard Zusman, Harold Dahlberg, William Johnson, and Harry Bradley of the Emergency Department, Norwalk Hospital, who provided medical supervision throughout testing;

- Mr. Richard Martel of Stephenson, Inc., and Mr. Manley Luckey of Luckey Laboratories, Inc., who furnished valuable technical information concerning the breath testing devices employed in this program. Special thanks are due to Stephenson, Inc., for furnishing spare test equipment without charge.*

- Mrs. Elizabeth King, Mr. Harold Smyth, and Mrs. Marlene Orban, colleagues at Dunlap and Associates, Inc., who served as Subject Escorts and data analysts.

Finally, most sincere thanks are due for the excellent cooperation offered by the thirty-seven program Subjects. Major credit for the success of this work quite clearly belongs to them.

\footnotetext{
*AII primary test equipment, including breath testing instruments was furnished by the Transportation Systems Center
} 
TABLE OF CONTENTS

Page

SUMMARY

I. BACKGROUND 2

A. Selected Devices 2

B. Experimental Objectives 2

C. Subjects 4

D. Program Phasing 7

E. Facilities, Equipment and Personnel 15

$\begin{array}{ll}\text { II. COMPLEX REACTION TESTER } & 17\end{array}$

$\begin{array}{ll}\text { A. Training Procedures } & 17 \\ \text { B. Testing Procedures } & 19 \\ \text { C. Analyses and Results } & 20\end{array}$

III. PHYSTESTER 33

A. Training Procedures 33

B. Testing Procedures 36

C. Analyses and Results 36

$\begin{array}{ll}\text { IV. QUICKEY } & 47\end{array}$

A. Training Procedures 47

B. Testing Procedures 49

C. Analyses and Results 51

$\begin{array}{lll}\text { V. REACTION ANALYZER } & 67\end{array}$

$\begin{array}{ll}\text { A. Training Procedures } & 67\end{array}$

B. Testing Procedures 68

C. Analyses and Results 69 
TABLE OF CONTENTS (Continued)

Page

VI. NARTRON

A. Training Procedures

79

B. Testing Procedures

VII. DISCUSSION

APPENDIX

A Observations of Physiological Phenomena

A - 1 Related to Alcohol

B Subject Selection Procedures

B-1 
LIST OF TABLES, FIGURES AND EXHIBITS

Table

Page

I Complex Reaction Tester Performance - Group I 26

II Complex Reaction Tester Performance - Group II 27

III Complex Reaction Tester Performance - Group III 28

IV Complex Reaction Tester Control Session Performance, 29 Group III

V Complex Reaction Tester Performance - Group IIIC 30

VI Distribution of Complex Reaction Tester Failed 31

VII Distribution of Complex Reaction Tester Failed 32

Trials - Control Sessions

VIII Phystester Performance - Group I 42

IX Phystester Performance - Group II 43

X Phystester Performance - Group III 44

XI Phystester Control Session Performance, Group III 45

XII Phystester Performance - Group IIIC 46

XIII Quickey Pass/Fail Criteria $\quad 50$

XIV Quickey Performance - Group I 61

XV Quickey Performance - Group II 62

XVI Quickey Performance - Group III 63

XVII Quickey Control Session Performance, Group III 64

XVIII Quickey Performance with Adjusted Criterion 65

XIX Quickey Performance - Group IIIC 66

XX . Reaction Analyzer Performance - Group II 76

XXI Reaction Analyzer Performance - Group III 77

XXII Reaction Analyzer Control Session Performance, 78 Group III 
XXIII Nartron Testing Criteria

A-I Observed Rates of Elimination, Program Subjects

A - 3

A-II Rates of Elimination - Coldwell and Grant Subjects

A -7

B-I Results of Pre-Test of Subject Screening Instrument

B -4

B - II

ASIS Test Subject Screening Scores and Peak BACs

B -6

B-III Alcohol Doses

B- 8

Figure

1 Complex Reaction Tester Performance - 3/3 Strategy

2 Complex Reaction Tester Performance - 2/2 Strategy

3 Complex Reaction Tester - Comparison of Drinking and

Control Sessions (Group III)

4 Phystester Performance - 2/3 Strategy

5 Phystester Performance - 2/2 Strategy

6 Phystester - Comparison of Drinking and Control

Sessions - 1/1 Strategy (Group III)

7 Quickey Performance - 1-16th Strategy

8 Quickey Performance - 1-20th Strategy

9 Quickey Performance - 1-24th Strategy

10 Quickey - Adjusted Criterion Performance, One Response

11 Quickey - Adjusted Criterion Performance, Two Responses

12 Quickey - Comparison of Drinking and Control Sessions 1-16th Strategy (Group III)

13 Reaction Analyzer Performance - 4/5 Strategy

14 Reaction Analyzer Performance - 2/3 Strategy 
LIST OF TABLES, FIGURES AND EXHIBITS, (Continued)

Figure

16

17

$A-1$

A -2

A - 3

\section{Exhibits}

I

II

III

IV

$B-I$
Reaction Analyzer Performance - 5/5 Strategy

Reaction Analyzer - Comparison of Control and

Drinking Sessions - 1/1 Strategy

Elimination Rate vs. BAC - Dunlap Data

Elimination Rate vs. BAC - Dunlap Data

Augmented by Coldwell and Grant (1963) Data

Theoretical Relationship Between BAC and Time (After Complete Absorption)
Page

73

75

A -5

A-8

A-9 $\approx$

Subject Admission Statement

Medical Examination Record

6

9

Slide Rule Used to Compute Alcohol Doses

11

Testing Time Line

13

Subject Screening Instrument

B- 9 


\section{SUMMARY}

This interim report describes the experimental program conducted by Dunlap and Associates, Inc. to investigate five candidate Alcohol Safety Interlock Systems. The program consisted of 114 Subject-days of experimentation designed to provide estimates of the performance of each device at blood alcohol concentrations up to and exceeding $0.18 \% \mathrm{wt}$. / vol.

The contents of the report may be summarized as follows:

- A definition of interlock performance is presented. This is tied to the proportion of drivers that a device would reject (i.e., prevent from driving) at various blood alcohol concentrations. Specific experimental objectives are derived from the overall goal of determining performance across a wide range of concentrations.

- Experimental procedures employed to satisfy the objectives are described in detail. These relate primarily to the selection, training, and testing of the 37 program Subjects, and to the conduct of analyses of test data.

- Detailed descriptions of each of the five interlocks are presented. Alternate design configurations under which each was tested are cited. Specific training and testing procedures applied to each are also stated. Equipment problems encountered are noted.

- Tabulations of performance as a function of blood alcohol concentration are presented for each device. Results are given in a manner permitting comparison of alternate design configurations, various implementation strategies, and different classes of Subjects.

- Conclusions are reached concerning the suitability of these instruments for future applications. Recommendations for additional investigations are also listed. 


\section{BACKGROUND}

This report, submitted to the U.S. Department of Transportation, Transportation Systems Center (TSC) under Contract DOT-TSC-251, documents the results of laboratory investigations of selected Alcohol Safety Interlock Systems (ASIS) conducted by Dunlap and Associates, Inc. during the period from March through June 1972. This program was designed to test the ability of each candidate ASIS to detect alcohol impairment among volunteer Subjects, and thereby indicate the instrument's suitability as a drinking-driving/countermeasure.

\section{A. Selected Devices}

ASIS units examined in this experimental program included:

- Complex Reaction Tester (DOT-TSC)
- Phystester (General Motors)
- Quickey (Robert D. Smith)
- Reaction Analyzer (Raytheon)
- The Nartron Device (Nartron, Inc.)

All five instruments conceptually belong to the psychomotor test class of interlock. That is, they do not chemically (or otherwise directly) measure blood alcohol concentration (BAC), but rather employ tests of coordination, memory, judgment, reaction time and/or other psychomotor faculties presumably influenced by alcohol. Each device poses a particular task, exercising some faculty or set of faculties, for which a pass/fail criterion may be defined. Inability to achieve this criterion causes the driver to be rejected (i.e., prevented from operating the vehicle).

Detailed descriptions of these devices, together with their respective tasks and pass/fail criteria, are presented in Section II through VI of this report.

\section{B. Experimental Objectives}

The basic goal of the program was to quantify the performance of the selected instruments across a wide range of $\mathrm{BAC}$, with particular emphasis on relatively high levels ( $\geq 0.15 \%$ wt. /vol.). As defined in a previous report, * ASIS

"Oates, J.F., Jr. and McCay, R. T. Methodologies for Estimating the Effectiveness of Alcohol Safety Interlock Systems. Report No. DOT-TSC-251-3, November, 1971. 
performance is the functional relationship the device exhibits between BAC and rejection (or "fail") rate. Previous efforts sponsored by TSC had provided performance estimates for some candidate instruments at low to moderate BAC, but few data were available at higher levels. Data in these higher ranges are especially critical since some sources* indicate that the average BAC of motor ists arrested for driving while intoxicated--a likely target population for ASIS application--exceeds $0.200 \%$ wt. / vol.

Within the context of this overall goal, the following specific objectives were pursued:

1. The selection of a sample of experimental Subjects, each of whom could be expected to attain these elevated levels of BAC;

2. The identification of a variety of potential implementation strategies for each candidate ASIS, against each of which performance could be measured. One such strategy, for example, might require the driver to pass at least two of a series of three trials in order to start his vehicle.

3. The identification and assessment of potentially beneficial modifications to the various ASIS units. The subdivision of the program into three distinct test periods (discussed in Subsection C of this Section) provided the opportunity to evaluate the relative merits of alternative design parameters affecting an instrument's degree of difficulty and/ or pass/fail criterion. Appropriate modifications were jointly agreed to by the project staff and the TSC Contract Technical Manager.

4. The provision of sufficient pre-test training on all devices to each Subject, to insure that adequate familiarity with the instruments had been achieved.

5. The design and implementation of a carefully controlled test with specific provisions for--

- The administration of precise doses of ethanol required to achieve desired levels of $B A C$

- Frequent monitoring of Subject BAC

"For example, the DOT-sponsored Alcohol Safety Action Projects. 
- The acquisition of sufficiently large samples of data, not only at high $\mathrm{BAC}$, but also at low to moderate levels to permit identification of extraneous effects and comparison with previous studies of this type

- Insuring high motivation among Subjects

- Protecting the health and safety of the Subjects

6. The application of suitable analytic techniques to derive and quantify ASIS performance.

In addition to the efforts expended to meet these objectives, several ancillary experiments were conducted in parallel. These included:

- Testing of various models of the Alcohol Screening Device (ASD), a portable breath testing instrument developed by DOT-TSC. The frequent monitoring of Subject $B A C$ by means of accurate, proven instruments provided numerous opportunities to conduct parallel measurements on the ASD. Data thus obtained have been submitted to the Contract Technical Manager in a separate memorandum.

- Investigations into the rates of absorption and elimination of blood alcohol, with particular regard to the possible effects of high BAC upon these rates. These topics are discussed in Appendix A.

- Collecting data concerning eye motion phenomena* as affected by BAC.

C. Subjects

Thirty-seven (37) Subjects, all licensed drivers, participated in this program. They were selected from among some sixty applicants on the basis of their suitability as manifested during a thorough, personall interview*;. They

* The eye motion study was conducted during the third test period. Each Subject sat in a fixed position at a chin rest and was required to track a laser-generated light spot undergoing sinusoidal and square wave horizontal excursions on a screen. A video-tape camera was focused on the Subject's eyes. During each test session, filming took place prior to ingestion of alcohol and subsequent to ingestion of most or all of the scheduled doses. Video tapes were submitted to DOT-TSC upon completion of testing.

***

Described in detail in Appendix B. 
ranged from 21 through 63 years of age; 20 were nules, 17 fromales; all were Caucasian.

A decision was made to select Subjects for whom there was a reasonable likelihood of previous--and preferably frequent--exposure to elevated BACs. This decision was predicated on the following two considerations:

- Such Subjects would be comparable to (and might include) members of a likely target population for ASIS implementation, i.e., individuals convicted of driving while intoxicated.

- The incidence of nausea, vomiting, and other undesirable effects that undoubtedly would contaminate assessment of ASIS performance could be expected to be lower among these individuals than among relatively "light" drinkers.

It could be argued that this purposely biased selection may have eliminated candidates who would exhibit pronounced impairment at low BAC, causing the program to produce a conservative estimate of ASIS performance. However, such candidates are felt to belong only rarely to the group of convicted drinking drivers who would be the target of a Limited ASIS application. With regard to Universal* application (for which the argument has greater validity), conservative performance estimates are perhaps prudent and desirable at this stage of ASIS development.

Subjects were recruited into the program during three distinct test periods. Group I (10 members) participated during March, Group II (12) during April, and Group III (15) during May-June. This phased approach permitted the conduct of intermediate analyses and the implementation of modifications to experimental procedures and ASIS units, as warranted.

Upon admission to the program, each Subject was required to sign a statement attesting to his voluntary participation, good health, understanding of the test requirements, and willingness to hold harmless Dunlap and Associates, Inc. and all its agents from any claims arising from his participation in the program. A copy of the statement is shown in Exhibit I.

The care devoted to Subject selection was considered absolutely essential to achieving the basic goal of the program. The success of this effort is re flected in the fact that, over the ninety-nine Subject-days of controlled drinking experimentation, the mean peak BAC exceeded $0.18 \%$. It is also worthy of note that there, were only ten instances when Subjects became ill to the point

i. e., installation of an ASIS in every new automobile, commencing with some specified model year. 


\section{EXHIBIT I}

SUBJECT ADMISSION STATEMENT

NAME

ADDRESS

AGE

TELEPHONE NUMBER

The undersigned hereby agrees to participate in the Dunlap and Associates Alcohol Research Program. Dunlap and Associates, Inc. has fully explained to me the nature, purpose and content of the program. I fully understand that I will voluntarily consume liquor and may become intoxiciated, and then submit to chemical and mechanical testing. I further understand that I may withdraw from participation in the program at any time. I represent that I have been advised by my physician that I am in good physical and mental health and have no history of health problems that would indicate that I should not participate in the program. I agree to release and hold harmless Dunlap and Associates, Inc., its agents, servants and employees, including the physicians making examinations on its behalf and/or monitoring this research program from any and all claims arising from my participation in or the conduct or the management of the program.

Signature 
of vomiting, notwithstanding the se elevated levels of blood alcohol.

\section{Program Phasing}

The program consisted of three major phases, which conveniently may be labelled Training, Testing, and Analysis of Performance. General descriptions of each phase are presented below.

\section{Training}

As applied to each Subject, the training phase consisted of a series of three sessions averaging roughly four hours duration each. Three to six Subjects participated in each session. The first session commenced with a detailed "hands on" demonstration of each ASIS unit conducted by a Dunlap staff member. Care was taken to insure that all Subjects fully understood the nature of the task and the proper manner of conducting a trial. Once this was accomplished, the Subjects were briefed on the major components of the training paradigm. These were:

- The Training block:

For each instrument, a specified number of trials were taken to constitute a single training block. Subjects were required to complete blocks in the specific order listed in the training booklet issued at the beginning of each session. This order dictated that the Subject repeatedly cycle through the ASIS devices on a block-by-block basis until all assigned work had been completed or until training criterion (defined below) was achieved.

- Training criterion:

Although a maximum number of blocks per training session was assigned for each ASIS, the Subject had an opportunity for early completion of a session's work on any given device. * To do so, he was required to achieve training criterion, defined as passing at least a specified number of trials (approximately 90-95\%) out of any given block or sequence of blocks. This opportunity was offered on each of the three training sessions.

Except for the Quickey for which a fixed number of blocks per session was required. 
- Buddy system:

Subjects worked in pairs on each training session. * When one Subject was undertaking an assigned block of trials, his partner recorded the results in the appropriate training booklet; once a block was completed, they exchanged roles. This scheme not only insured orderly progression through the ASIS devices, but also enforced frequent rest periods for each Subject, thus minimizing fatigue. A light meal midway through the session provided another rest period of longer duration.

- Reward system:

Subjects received $\$ 25$ base pay for attendance at each training session. In addition, incentive payments were issued for achieving training criterion. This was done to maintain high motivation and thus, hopefully, to accelerate the "learning curve." It was fairly common for Subjects of the first two groups--who had the opportunity to achieve incentive payment on the Quickey--to amass rewards exceeding the base pay.

\section{Testing}

The testing phase for each Subject likewise consisted of a series of three sessions, of roughly seven hours duration each. Three to five Subjects participated in each session. The schedule was so designed that no Subject participated on consecutive days.

The major components of a testing session are described below:

- Medical examination:

At the beginning of each session, Subjects received a brief medical examination conducted by the attending physician to insure that no impediments to their participation existed. Occasional re-examinations were conducted during the sessions whenever the physician deemed necessary. A copy of the medical examination record is shown as Exhibit II.

\footnotetext{
* For those sessions during which an odd number of Subjects trained, a Dunlap staff member served to round out a team.
} 


\section{EXHIBIT II}

MEDICAL EXAMINATION RECORD

$\Xi$

$=$

Name:

Height:

Address:

Weight:

Age:

Sex: $\quad M \quad F$

Phone:

Pulse:

Blood Pressure:

Heart:

Temp:

Respiration:

Color:

Examination Date:

Subject is / is not qualified to participate in controlled drinking study. Examining Physician: 


\section{- Administration of alcohol:}

Each Subject was scheduled to imbibe four drink: during the course of the session. These consisted of measured volumes of 190 proof grain alcohol mixed with the Subject's choice of fruit juice. The volume of alcohol assigned to each drink was based on the Subject's weight and observed rate of absorption. A circular slide rule developed by the Charlotte-Mecklenburg (N. C.) Alcohol Safety Action Project and based upon Widmark's $\underline{R}$ was used to compute the required dosage (see Exhibit III). Typical target BACs for each drink are listed below:

Drink \# 1

Drink \#2

Drink \# 3

Drink \#4
$0.04 \%-0.06 \%$

$0.08 \%-0.11 \%$

$0.12 \%-0.16 \%$

$0.16 \%-0.2 .0 \%$

Drinking and waiting periods:

Fifteen minutes were devoted to the ingestion of each drink. This was followed by a twenty minute waiting period to allow for absorption of alcohol into the blood stream and dissipation of alcohol from the mucous membranes of the mouth; at the end of this period the Subject was required to rinse his mouth with water to further insure elimination of residual alcohol.

Subjects were permitted to play cards, read magazines, and take part in similar diversions during the drinking/waiting periods in order to maintain a relaxed, comfortable atmosphere throughout the testing. Smoking was permitted during the drinking period and through roughly the first fifteen minutes of the waiting period. Noeating whatsoever was allowed during these times.

Test cycles:

All testing took place during discrete cycles consisting of the following events:

- submission to a breath test

- completion of a block of trials on ASIS device \#1

- completion of a block of trials on ASIS device \#2 


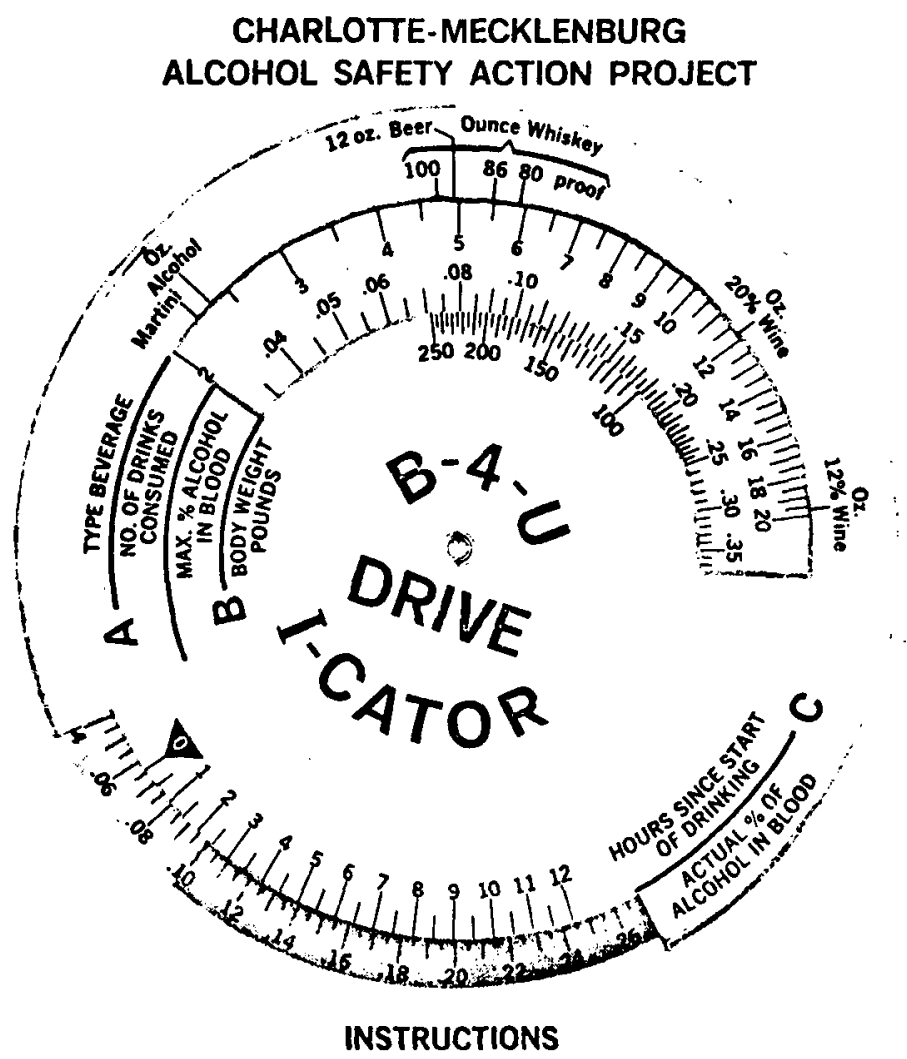

A. Align number of drinks consumed with type of beverage consumed.

B. Above your body weight, read maximum percentage of alcohol possible in blood.

C. Align the number of hours since start of drinking over. the maximum percentage of alcohol in blood (as found in $B$ ) and then read actual percentage of blood alcohol under arrow. 
- submission to a breath test

- completion of a block of trials on ASIS device \#3

- completion of a block of trials on ASIS device \#4

- submission to a breath test

Each cycle was of approximately 25 minutes duration. A typical session consisted of nine cycles, distributed approximately along the time line shown in Exhibit IV.

Control data:

Test cycles preceding ingestion of the first drink were intended to provide data concerning ASIS performance at zero BAC, an essential factor to consider in assessing the effectiveness of the devices. However, it was recognized that these data:

- Might be contaminated by the natural anxiety present among certain Subjects at the beginning of a test session

- Might not adequately serve to indicate fatigue effects.

In order to isolate such factors, Group III Subjects each participated in one control session. These were identical to the standard sessions, except that measured amounts of water replaced the corresponding volumes of alcohol in the drinks administered; all other factors, including specifically the ingestion of liquids and observation of waiting periods, remained the same. Control sessions were isolated events, i. e., three such sessions were conducted with all participating Subjects abstaining from alcohol.

- Reward system:

As in the case of the training phase, Subjects received $\$ 25$ base pay for each testing session. In addition, a reward was given for each ASIS trial passed. This was done to simulate the motivation a driver would experience if passing the trial were a prerequisite to starting his car. Subjects received their rewards in the form of poker chips immediately upon the completion of each test cycle. The spirit of competition this fostered seemed to further enhance motivation. 


\section{EXHIBIT IV}

\section{TESTING TIME LINE}

Time $=0$

\section{$10 \mathrm{~min}$}

\section{Medical Examination}

$35 \mathrm{~min}$

$$
\text { Cycle \# } 1
$$

$$
\text { Cycle \#2 }
$$

1 hour $0 \mathrm{~min}$

1 hour $15 \mathrm{~min}$

1 hour $35 \mathrm{~min}$

2 hours $0 \mathrm{~min}$

2 hours $15 \mathrm{~min}$

2 hours $35 \mathrm{~min}$

3 hours $0 \mathrm{~min}$

3 hours $15 \mathrm{~min}$

3 hours $35 \mathrm{~min}$

4 hours $0 \mathrm{~min}$

4 hours $15 \mathrm{~min}$

4 hours $35 \mathrm{~min}$

5 hours $0 \mathrm{~min}$

Ingestion of first drink

Waiting period

Cycle \#3

Ingestion of second drink

Waiting period

$$
\text { Cycle \#4 }
$$

Ingestion of third drink

Waiting period

Cycle \#5

Ingestion of fourth drink

Waiting period

Cycle \#6

Cycle \#7

5 hours $25 \mathrm{~min}$

6 hours $0 \mathrm{~min}$

6 hours $30 \mathrm{~min}$

7 hours $0 \mathrm{~min}$
Light meal/rest period

Cycle \#8

Cycle \#9 


\section{Analysis of Performance}

The performance of an ASIS will depend upon the strategy under which it is implemented. The simplest strategy is one in which the outcome of a single rrial determines whether the vehicle will be started. More complex strategies could permit the driver to attempt a set of trials, some subset of which must be passed if the car is to start. For purposes of this report, the general form of an ASIS strategy is represented by $N / M$, where $M$ is the number of attempts allowed and $\mathrm{N}$ is the minimum number which must be passed if a "START" is to be recorded.

Since testing of the devices selected for this program employed modular blocks of trials, it was possible to evaluate each under a number of different strategies. First, each block of $\underline{M}$ trials was viewed as a unit, permitting analysis of performance with respect to strategies ranging from $1 / M$ to $M / M$. Second, by treating the first N trials in each block as a discrete unit (subblock), similar strategies with fewer attempts permitted could be examined. Finally, by treating each individual trial as an independent unit, performance could be estimated with respect to a simple $1 / 1$ strategy.

Analysis of performance under each strategy consisted of the computation of the percentage of test units (blocks, subblocks, or trials) rated as rejections ("fails to start") at each interval of BAC. Ideally, one would wish to treat $B A C$ as a continuum for such analysis; however, sample size limitations necessitated the adoption of BAC class intervals. Both to insure adequate representation in each interval and to permit comparison with previous studies funded by TSC, the following class intervals were employed:

$$
\begin{aligned}
& \text { 1. } 0.000 \%-0.029 \% \\
& \text { 2. } 0.030 \%-0.059 \% \\
& \text { 3. } 0.060 \%-0.089 \% \\
& \text { 4. } 0.090 \%-0.119 \% \\
& \text { 5. } 0.120 \%-0.149 \% \\
& \text { 6. } 0.150 \%-0.179 \% \\
& \text { 7. } 0.180 \% \text { and above. }
\end{aligned}
$$

Each testing block was assigned to a particular interval in accordance with the breath tests results obtained during the test cycle in which the block was taken. Control data obtained from the Group III Subjects were also analyzed with respect to the various strategies applied to"each device. Performance estimates resulting from these data were examined as a function of elapsed test session time rather than $B A C$. 
One other factor considered in these analyses was the variation in ASIS performance exhibited by the different classes of Subjects. All strategies were examined independently for males, females, young Subjects (age $<30$ ), and older Subjects (age $\geq 30$ ), and each of the three groups.

Detailed discussions of the training, testing, and analysis applicable to the five ASIS units selected for this program are presented in Sections II through VI. Compilations of training and testing data for each Subject on each ASIS have been submitted to the Contract Technical Manager and are on file at TSC.

\section{E. Facilities, Equipment, and Personnel}

All training and testing sessions took place in a suite of rooms located in an isolated wing of the Dunlap and Associates, Inc. headquarters in Darien, Connecticut. These facilities permitted each ASIS device to be located in a separate room, thus allowing simultaneous training/testing of two or more Subjects. The suite also included a spacious, carpeted, and well-ventilated lounge area conducive to the maintenance of a relaxed, pleasant atmosphere; Subjects remained in this lounge during drinking and waiting periods. Additional rooms were set aside for the medical examinations, materiel storage, and the conduct of breath tests.

Apart from the ASIS devices, the major equipment items employed in this program were two breath testing instruments, the Alco-Analyzer Gas Chromatograph* and the Breathalyzer, ** Model 900. The Gas Chromatograph was employed for the breath tests taking place at the beginning and end of each test cycle, the Breathalyzer for the mid-cycle test. Several days were devoted to conducting breath alcohol simulator tests of both instruments. The Gas Chromatograph, which produces a graphic output rather than direct, numerical values of $\mathrm{BAC}$, was found to provide highly repeatable measurements. Using simulator solutions of $0.025 \%, 0.05 \%, 0.10 \%, 0.15 \%, 0.20 \%$, and $0.25 \%$, a linear relationship was found to exist between $\mathrm{BAC}$ and the peak height of the graphic output. This relationship, described by the following equation,

$$
\mathrm{BAC}=(.0031) \mathrm{H}+.019
$$

(Eq. $1 * * * *)$

*Luckey Laboratories, Inc., San Bernadino, California.

$* *$

Stephenson, Inc., Eatontown, New Jersey.

**** Where $\mathrm{H}$ is the peak height (in scale divisions) of the alcohol curve; note that BACs lower than $0.019 \%$ cannot be measured, apparently due to a bias in the graphical recording subsystem. 
was used to compute the Subject's blood alcohol equivalent after each Gas Chromatograph breath test. The Breathalyzer, which does provide direct readings of $B A C$, was found to produce generally consistent but slightly erroneous readings. The following equation was derived from the simulator test data and was used to analytically "filter" the error:

$$
\mathrm{BAC}_{\mathrm{T}}=1.06\left(\mathrm{BAC}_{\mathrm{R}}\right)-.003
$$

All Breathalyzer measurements therefore were adjusted on this basis.

During a portion of the third group's testing, the Gas Chromatograph was inoperative due to a malfunctioning breath column. Until that component was replaced, only the Breathalyzer was a vailable for monitoring BAC. This situation applied to five testing sessions.

Control of all testing sessions was exercised by the Project Director, who conducted all breath tests, assigned the magnitude of each alcohol dosage, and insured adherence to the testing schedule. Two or three staff members served as Subject Escorts during each session. In addition to recording the results of all ASIS trials and breath tests, their duties included mixing and administering drinks, transporting Subjects to and from drinking sessions, and providing close observation of Subjects to protect their safety. Finally, one physician ${ }^{*} * w^{2}$ attended each drinking session. His duties included conducting the medical examinations and protecting the general health and safety of all Subjects.

\footnotetext{
"Where $\mathrm{BAC}_{\mathrm{R}}$ is the instrument's "Raw" measurement arid BAC $\mathrm{T}$ is the adjusted value.

*** Medical support was supplied by the staff of the Emergency Department, Norwalk (Connecticut) Hospital.
} 


\section{COMPLEX REACTION TESTER}

The Complex Reaction Tester, developed by DOT-TSC, employs a twoby-two array of indicator lights arranged as the corners of a rectangle whose horizontal dimension is much greater than the vertical. A pushbutton is mounted below each vertical pair of lights. During the course of a trial these lights illuminate in an unpredictable sequence for a total of eight flashes, occurring roughly 10 seconds apart. When an upper light flashes, the Subject must respond by depressing the pushbutton on the same side of the rectangle as the stimulus; when the lower light flashes, he must depress the pushbutton on the opposite side.

The allowable response time for a single flash is selectable from among the following set: 0.9 seconds, 1.8 seconds, or 2.6 seconds; flash duration is roughly equivalent to selected response time. The Subject commits an error by either failing to respond within the allowable time or by failing to depress the correct pushbutton. Commission of more than the allowable number of errors (selectable as 0,1 , or 2) over the course of the eight flashes results in failure of the trial. A distinct combination of response time setting and error tolerance was employed for each of the three groups of Subjects.

"Pass" or "Fail" of a Complex Reaction Tester trial is displayed by green and red indicator lights respectively flanking a white "TEST" light. Originally, neither indicator illuminated until all eight flashes were completed. A modification incorporated for Group III provided immediate indication of "FAIL" upon commission of more than the allowable number of errors.

Details of the training, testing, and analyses applied to the Complex Reaction Tester are set forth below.

\section{A. Training Procedures}

Complex Reaction Tester training varied in accordance with the discrete permutations of response time setting and error tolerance applied to the three groups. The permutation used for Group I permitted one error and had variable response time. Subjects trained for up to 4 blocks of 4 trials on each of the three training sessions. On the first session, training started at a response time of 2.6 seconds. As soon as a trial was passed at this speed, response time was reduced to 1.8 seconds. Similarly, when a trial was passed at 1.8 seconds, response time was again reduced to the minimum value of 0.9 seconds. Once the slow and intermediate levels were passed, all subsequent training (and testing as well) was carried out at the 0.9 second setting. 
The Subject earned a $\$ 5$ reward when he accomplished a complete block of 4 trials at 0.9 seconds response time without a failure. Once this training criterion was achieved, no further trials were taken during that particular session. Subjects worked toward criterion (4 passes in a single block) and the $\$ 5$ reward on each of the three training sessions. Of the 10 Subjects in Group I, 6 reached criterion on the first session of training, 7 on the second, and 9 on the third. The one Subject who did not reach criterion on his third session had done so on the second. Several Subjects were given more than the scheduled 4 blocks (16 trials) in a given session when they were not able to reach criterion in the normal number. The maximum number of trials on any given session was 24 which occurred once. The minimum was 4 which occurred 7 times in the 30 SubjectDays of Group I training. The mean numbers of trials per Subject over the three training sessions were 16.4, 9.6, and 11.7 respectively per session with an overall mean of 37.7 trials per Subject.

The permutation of the Complex Reaction Tester used for Group II had a fixed response time and a selectable tolexance for 2, 1 or 0 errors. The exact response time was not stated, but investigation by project staff indicated flash duration to be approximately 1.5 seconds. (Since response time closely approximated flash duration, it is assumed that the unspecified response time was 1.8 seconds as indicated above.) The basic training design was similar to that for Group I with a schedule of up to 4 blocks (of 4 trials) on each of three sessions. On the first session, the Subject worked at the "2 errors permitted" setting until he passed once, then moved to the "1 error permitted" until one trial was passed. Once the 2 and 1 error conditions were passed, all subsequent training was carried out at the 0 error setting.

The Subject earned a $\$ 5$ reward if he achieved 4 passes in a single block at the 0 error setting. The achievement of this criterion terminated training for that session, but eligibility for the reward was renewed on each subsequent training session. Of the 12 Subjects, 11 achieved criterion on the first and all 12 succeeded on their second and third sessions. No Subject received more than the scheduled 16 trials per session. This was the maximum number and was received on 3 of the 36 Subject-Days of training. The minimum was 4 trials and was experienced on 20 of the 36 Subject-Days. The mean numbers of trials per Subject over the three training sessions were $11.3,5.0$ and 4. 3 respectively per session with an overall mean of 20.7 trials per Subject.

A comparison of the training data from Groups $I$ and II indicates that the second permutation of the Complex Reaction Tester (1.8 second response time with 0 errors permitted) was an appreciably easier task than was the first (0.9 second response time, 1 error). As noted in sub-section $C$ of this section, this observation was borne out in testing as well. The alternative explanation of the Group II Subjects being more competent should also be considered. 
The Group III permutation also held response time fixed while permitting the number of errors to be varied from 2 to 0 . The response time was fixed at the 0.9 second setting, however, which had been the primary training and testing mode for Group I. A further variation was that the "Fail" indication was displayed immediately upon the occurrence of the error leading to a failure. The basic training design was that which had been used previously (up to 4 blocks of 4 trials on each of 3 training sessions). Group III, however, faced a different criterion for the $\$ 5$ reward. This criterion was 7 passes out of 8 trials achieved in 2 consecutive blocks at the 0 error setting. This procedure assured more extensive practice than had either of the preceding methods. The absolute minimum number of trials possible under this design was 12 on the first session (since the first block necessarily started at the 2 error setting and therefore did not contribute toward training criterion) and 8 on the second and third sessions.

The added difficulty of the combination of this equipment permutation and criterion format is evidenced by the fact that only 1 of the 15 Subjects in Group III achieved training criterion on his first session. This rate of $6.7 \%$ compares with $60 \%$ for Group I and $91.7 \%$ for Group II. On the second training session, 13 of the 15 Subjects achieved criterion and 14 did so on the third. The individual failing on the third session had succeeded on the second.

The mean numbers of trials per subject over the three training sessions was up substantially from Group II to 16.0, 13.9, and 10.0 respectively with an overall mean of 40.0 trials per Subject. This was a slight increase over the amount of practice acquired by Group I as well.

\section{B. Testing Procedures}

Testing for all three groups of Subjects was based upon a modular block of three trials. For Group I, the operating parameters were 0.9 second response time and 1 error permitted. A total of 30 Subject-Days of testing were accomplished. For Group II, testing was started using both the 1 error and the 0 error settings and a response time of 1.8 seconds (i. e., two blocks were completed during each test cycle; these were not taken consecutively). After 20 Subject-Days of testing, the 1 error setting was discontinued as a result of an experience of $100 \%$ passes regardless of BAC up to and including levels in excess of $0.200 \%$. Testing at the 0 setting continued for the remaining 13 Subject-Days of Group II. Group III tested at 0 errors and 0.9 second response time and provided 45 Subject-Days of data (including control sessions). 
Groups I and II were rewarded at the rate of $\$ 0.50$ per successful pass. Group III operated under a different reward system, based upon $\$ 0.25$ per pass with a $100 \%$ bonus if all trials of the block were passed. While the total possible reward per block was unchanged, it was felt that this latter procedure would more effectively maintain motivation than had the uniform pay-off mode. No specific test of the relative strength of motivation was made.

Certain equipment failures were experienced during testing of the Complex Reaction Tester. At several points during Group II testing; the flash sequence would not start until the equipment was totally powered-down and re-started. At another point, the rate of flashes speeded up and their sequence became totally predictable. During Group III testing, the failures to start occurred quite frequently. The cause was isolated near the end of testing as being static electricity charges generated by the Subject moving across the carpeted test area. Emplacement of a sheet of hardboard under the testing table and the Subject's chair appeared to remove the problem. Similar sensitivity in an automobile where static charges are frequently encountered could be a severe operational problem.

During testing of Groups I and II, the Escort recorded merely "pass" or "fail" for each trial of the block. For Group III, the number and location of the flash on which an error was made were noted as well for all failed trials.

As a small scale test of the relationship of three permutations tested, 2 Subjects from Group I and 1 Subject from Group II were re-tested on the Complex Reaction Tester with the Group III design parameters without having undergone a significant amount of retraining. * (Two or three familiarization trials were permitted until the Subject acknowledged that he was satisfied that he remembered the task adequately.)

\section{Analyses and Results}

Strategies for which Complex Reaction Tester performance was analyzed included:

$$
\begin{aligned}
& \text { "one-out-of-one" } \\
& \text { "three-out-of-three" } \\
& \text { "two-out-of-three" } \\
& \text { "two-out-of-two" }
\end{aligned}
$$

*These Subjects were participating in the special Quickey experiment described in Section IV of this report. 
Results are depicted in Tables I, II, and III for Groups I, II, and III, respectively. Control session data* obtained from Group III are shown in Table IV; results obtained from the three Subjects participating in the special Quickey experiment (Group IIIC) are shown in Table V.

It is obvious that the various design permutations of response time and error tolerances strongly affected Complex Reaction Tester performance. The 1.8 second response time to which Group II was exposed resulted in generally low rejection rates at high $\mathrm{BAC}$, even under such relatively exacting strategies as $2 / 2$ and $3 / 3$. Reduction to 0.9 seconds considerably increases these rates, especially when coupled with 0 error tolerance. However, all design modes and all strategies exhibit undesirably high rejection rates at very low BACs and substantial pass rates at very high levels (see Figures 1 and 2). Results thus seem to indicate that the Complex Reaction Tester is inherently a fairly difficult task that is only moderately sensitive to BAC. However, the apparent degree of difficulty might well reflect the Subject's attitude toward this device as much as or more than the actual rigor of the task. Subjects were virtually unanimous in their dislike of the instrument. The relatively long duration of each trial, the need for nearly constant concentration, and the utter frustration associated with an eventual failure indication after responding to all eight stimulus flashes** produced tension among nearly all Subjects, mild repugnance among many others, and thinly veiled hostility among some. The importance of this attitude--which no other ASIS unit seemed to generate--should not be overlooked when considering the possibility of operational applications of the device. The incidence of tampering, removal, destruction, and employment of any and all overt or covert methods of circumventing the ASIS undoubtedly will increase as a function of the driver's degree of dislike for the instrument. This incidence can be expected to be very high for the Complex Reaction Tester.

Performance data corresponding to the $3 / 3$ strategy were subjected to

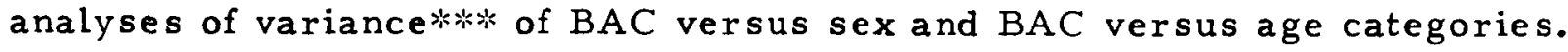
Results from all three groups (listed below) exhibit no significant differences with respect to sex and age $(p>.05)$.

* Subject \#130 exhibited BAC in excess of $0.04 \%$ at the beginning of his control session; his data are not included in the tabulations of control session results presented in this report. *k*k

Group III Subjects seemed somewhat less hostile toward the Complex Reaction Tester than did their earlier counterparts, possibly because failure was immediately indicated when a response was in error.

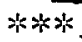

Performance data are proportional in nature, and so were subjected to arcsine transformation prior to analysis of variance. This procedure is suggested in Snedecor, G. W. Statistical Methods, Iowa State University Press, $1956 ;$ pg. 316. 


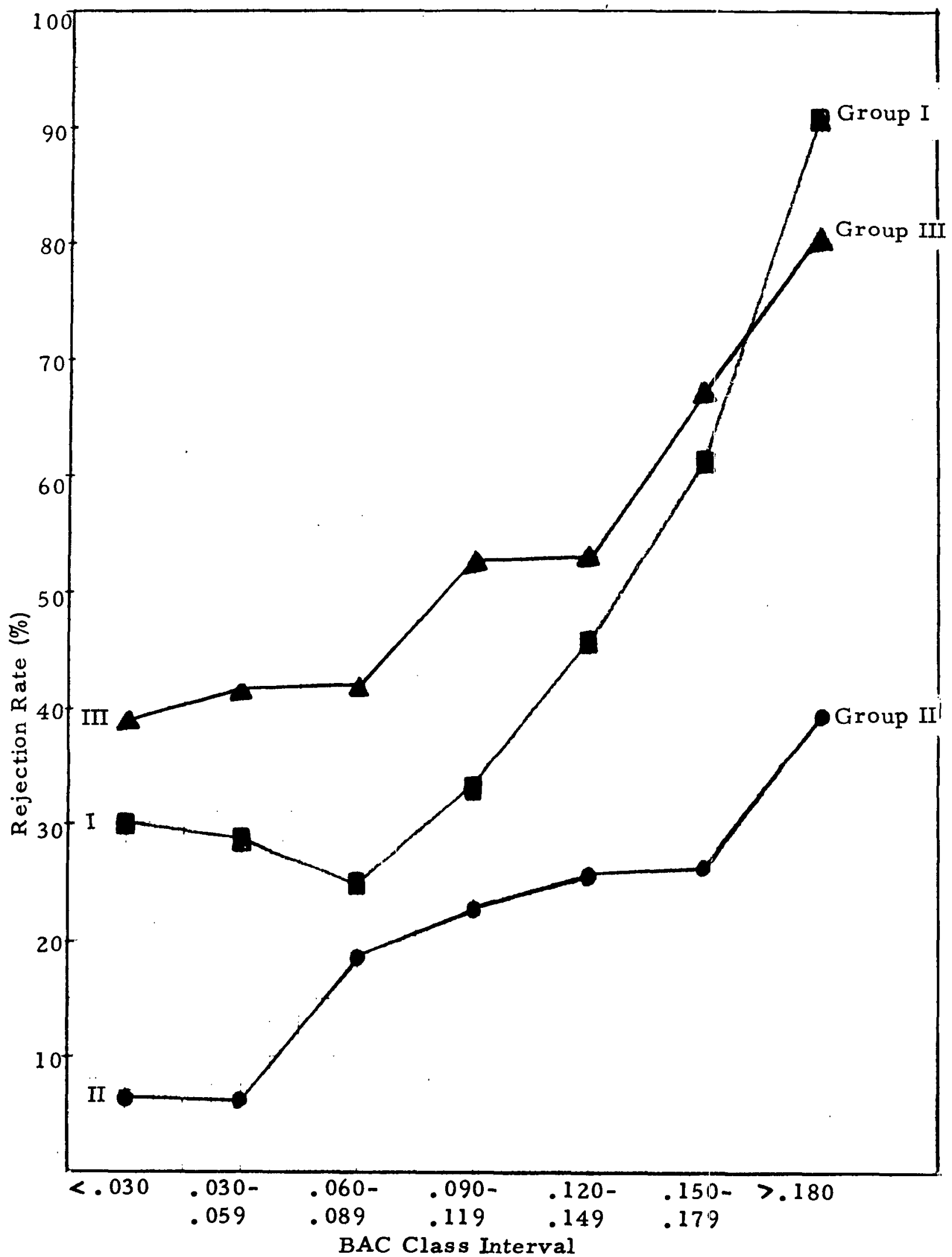

Figure 1. Complex Reaction Tester Performance - 3/3 Strategy 


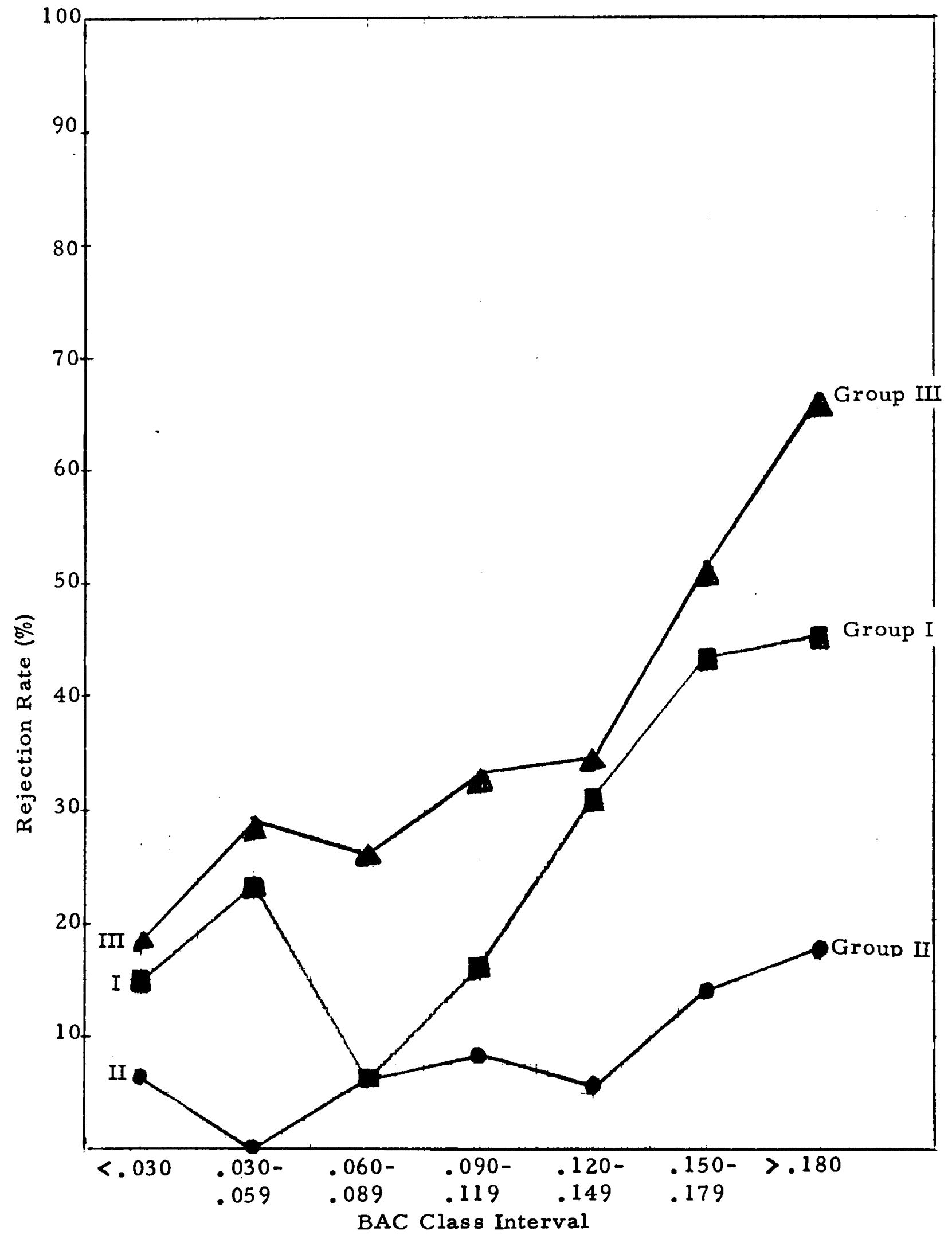

Figure 2. Complex Reaction Tester Performance - 2/2 Strategy 


\begin{tabular}{c|c|c|c|c|c|}
\cline { 3 - 6 } \multicolumn{2}{l|}{} & BAC & SEX & BAC & AGE \\
\hline $\begin{array}{c}\text { Group } \\
\text { I }\end{array}$ & $\begin{array}{c}F \\
\text { Significance }\end{array}$ & $\begin{array}{c}4.91 \\
.05>\mathrm{p}>.01\end{array}$ & $\begin{array}{c}0.64 \\
\text { N.S. }\end{array}$ & $\begin{array}{c}4.29 \\
.05>\mathrm{p}>.01\end{array}$ & $\begin{array}{c}4.25 \\
10>\mathrm{p}>.05\end{array}$ \\
\hline $\begin{array}{c}\text { Group } \\
\text { II }\end{array}$ & $\begin{array}{c}\mathrm{F} \\
\text { Significance }\end{array}$ & $\begin{array}{c}2.52 \\
.20>\mathrm{p}>.10\end{array}$ & $\begin{array}{c}2.31 \\
.20>\mathrm{p}>.10\end{array}$ & $\begin{array}{c}17.95 \\
.01>\mathrm{p}>.001\end{array}$ & $\begin{array}{c}3.79 \\
.10>\mathrm{p}>.05\end{array}$ \\
\hline $\begin{array}{c}\text { Group } \\
\text { III }\end{array}$ & $\begin{array}{c}\mathrm{F} \\
\text { Significance }\end{array}$ & $\begin{array}{c}3.45 \\
.10>\mathrm{p}>.05\end{array}$ & $\begin{array}{c}5.15 \\
.10>\mathrm{p}>.05\end{array}$ & $\begin{array}{c}55.57 \\
.001>\mathrm{p}\end{array}$ & $\begin{array}{c}0.33 \\
\text { N.S. }\end{array}$ \\
\hline
\end{tabular}

The immediate failure indication presented to the Group III Subjects permitted collection of more detailed data concerning each failed trial. Table VI presents distributions of the flash stimulus locations and numbers which led to failures at each BAC interval. Table VII exhibits similar distributions of control session data. Chi-squared tests indicated no significant relationship between BAC and the locations or numbers of stimulus flashes at which failures occurred.

Examination of control session data discloses appreciable fluctuations in rejection rate as a function of test session time. The rate was initially relatively high, decreasing markedly on cycle number 3. A plausible explanation might be that Subjects feel some anxiety at the beginning of a test session, which dissipates once they have settled into the test routine. This possibility could also account for the decrease in rejection rate sometimes noted during drinking sessions at low levels of BAC (typically, over the range from $0.03 \%$ to $0.05 \%$ ). From cycles number 3 through number 7 , the percent of trials failed during control sessions increased fairly steadily, which may indicate that fatigue and/or the sense of frustration mentioned previously had an effect. The drop in rejection rate during cycles 8 and 9 may be due to the beneficial effects of the light meal/rest period that pre ceded that period of the session or may reflect an "end spurt" commonly found in human response studies when the Subject knows the session is nearly over.

Figure 3 depicts the proportion of Complex Reaction Tester trials failed by Group III for both drinking and control sessions on a cycle-by-cycle basis. The mean BAC of each drinking session cycle is also given. Analysis of variance on these data indicates that the main effect for Treatment (Control vs. Drinking) is significant $(F=18.47, p<.01)$ while that for Cycle is not. It is apparent from the curves in Figure 3 that there is an appreciable interaction (treated as the "error" term in this analysis) which tends to mask any cycle effects which may exist and which is not itself testable. 


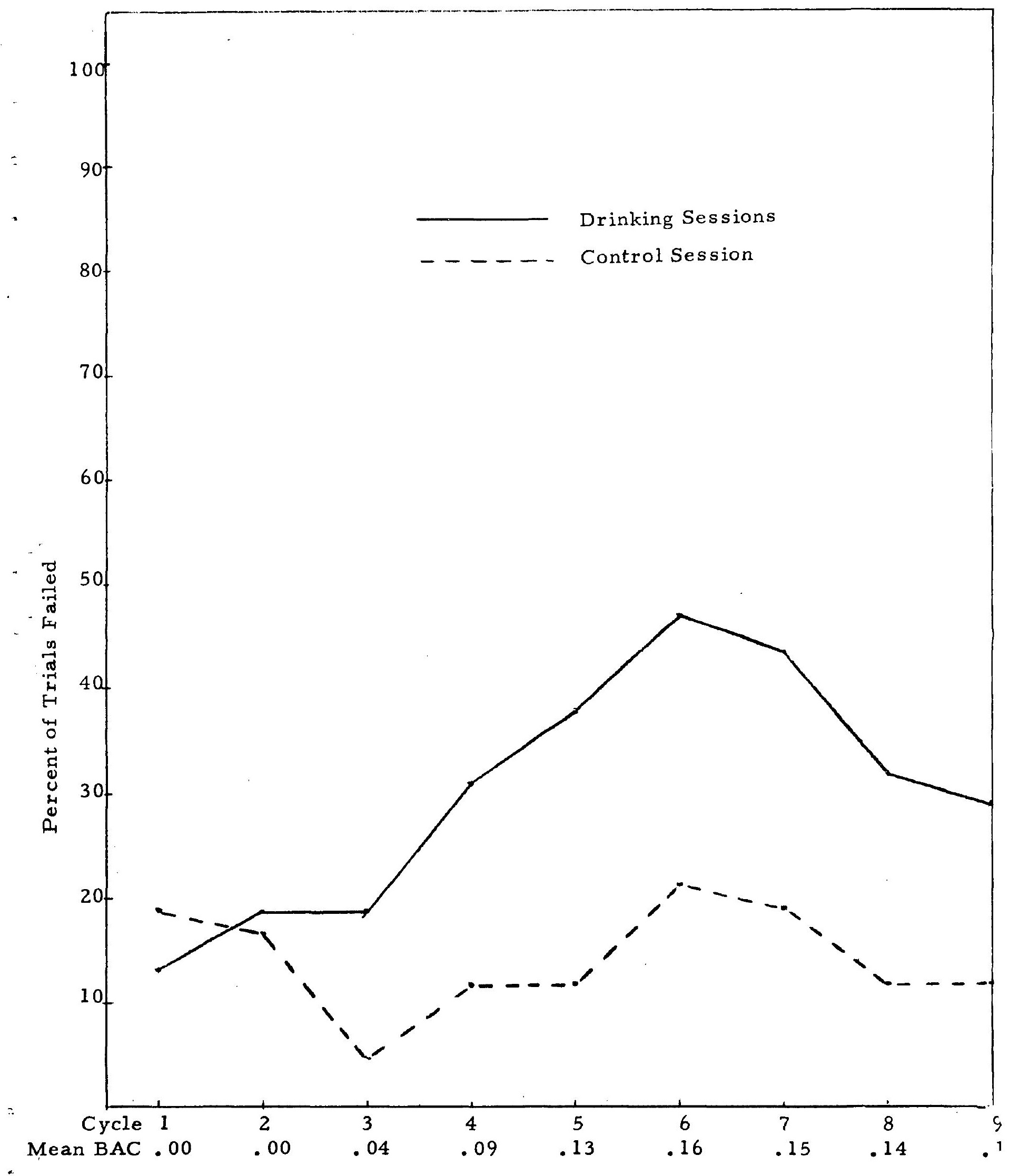

Figure 3. Complex Reaction Tester - Comparison of Drinking and Control Sessions - Group III 
Table I

\section{Complex Reaction Tester Performance, Group I $R$ esponse time: 0.9 seconds Error tolerance: 1}

STRA TEGY

\begin{tabular}{|c|c|c|c|c|c|c|c|c|c|}
\hline $\begin{array}{l}\text { SUBJECT } \\
\text { CATEGORY }\end{array}$ & $\begin{array}{l}\text { BAC CLASS } \\
\text { INTERVAL }\end{array}$ & \multicolumn{2}{|c|}{$1 / 1$} & \multicolumn{2}{|c|}{$3 / 3$} & \multicolumn{2}{|c|}{$2 / 3$} & \multicolumn{2}{|c|}{$2 / 2$} \\
\hline \multirow{7}{*}{$\begin{array}{l}\text { ALL } \\
\text { SUBJECTS }\end{array}$} & $<0.030 \%$ & $19 / 120$ & 15.8 & $12 / 40$ & 30.0 & $4 / 40$ & 10,0 & $6 / 40$ & 15.0 \\
\hline & $0: 030-0.059 \%$ & $10 / 63$ & 15.9 & $6 / 21$ & 28.6 & $3 / 21$ & 14.3 & $5 / 21$ & 23.8 \\
\hline & $0.060-0.089 \%$ & $4 / 48$ & 8.3 & $4 / 16$ & 25.0 & $0 / 16$ & 0.0 & $1 / 16$ & 6.2 \\
\hline & $0.090-0.119 \%$ & $13 / 90$ & 14.4 & $10 / 30$ & 33.3 & $3 / 30$ & 10.0 & $5 / 30$ & 16.7 \\
\hline & $0.120-0.149 \%$ & $37 . / 144$ & 25.7 & $22 / 48$ & 45.8 & $11 / 48$ & 22.9 & $15 / 48$ & 31.2 \\
\hline & $0.150-0.179 \%$ & $65 / 171$ & 38.1 & $35 / 57$ & 61.4 & $20 / 57$ & 35,1 & $25 / 57$ & 43.2 \\
\hline & $\geq 0.180 \%$ & $31 / 66$ & 47.0 & $20 / 22$ & 90.9 & $8 / 22$ & 36.4 & $10 / 22$ & 45.5 \\
\hline \multirow{7}{*}{ MALES } & $<0.030 \%$ & $6 / 54$ & 11.1 & $4 / 18$ & 22.2 & $1 / 18$ & 5.6 & $3 / 18$ & 16,7 \\
\hline & $0.030-0.059 \%$ & $3 / 30$ & 10.0 & $2 / 10$ & 20.0 & $1 / 10$ & 10.0 & $2 / 10$ & 20.0 \\
\hline & $0.060-0.089 \%$ & $2 / 27$ & 7.4 & $2 / 9$ & 22.2 & $0 / 9$ & 0.0 & $1 / 9$ & 11.1 \\
\hline & $0.090-0.119 \%$ & $4 / 33$ & 12.1 & $3 / 11$ & 27.3 & $1 / 11$ & 9.1 & $1 / 11$ & 2.1 \\
\hline & $0.120-0.149 \%$ & $11 / 69$ & 15.9 & $6 / 23$ & 26.1 & $3 / 23$ & 13.1 & $5 / 23$ & 21.7 \\
\hline & $0.150-0.179 \%$ & $24 / 93$ & 25.8 & $15 / 31$ & 48.4 & $7 / 31$ & 22.6 & $10 / 31$ & 32.3 \\
\hline & $=0.180 \%$ & $14 / 30$ & 46.7 & $10 / 10$ & 100.0 & $3 / 10$ & 30.0 & $4 / 10$ & 40.0 \\
\hline \multirow{7}{*}{ FEMALES } & $<0.030 \%$ & $13 / 66$ & 19.7 & $8 / 22$ & 36.4 & $3 / 22$ & 13.6 & $3 / 22$ & 13.6 \\
\hline & $0.030-0.059 \%$ & $7 / 33$ & 21.2 & $4 / 11$ & 36.4 & $2 / 11$ & 18.2 & $3 / 11$ & 27.3 \\
\hline & $0.060-0.089 \%$ & $2 / 21$ & 9.5 & $2 / 7$ & 28.6 & $0 / 7$ & 0.0 & $0 / 7$ & 0.0 \\
\hline & $0.090-0.119 \%$ & $9 / 57$ & 15.8 & $7 / 19$ & 36.8 & 2/19: & 10.5 & $4 / 19$ & 21.1 \\
\hline & $0.120-0.149 \%$ & $26 / 75$ & 34.7 & $16 / 25$ & 64.0 & $8 / 25$ & 32.0 & $10 / 25$ & 40.0 \\
\hline & $0.150-0.179 \%$ & $41 / 78$ & 52.6 & $20 / 26$ & 76.9 & $13 / 26$ & 50.0 & $15 / 26$ & 57.7 \\
\hline & $\geq 0.180 \%$ & $17 / 36$ & 47.2 & $10 / 12$ & 83.3 & $5 / 12$ & 41.7 & $6 / 12$ & 50.0 \\
\hline \multirow{7}{*}{$\begin{array}{l}A G E \\
<30\end{array}$} & $<0.030 \%$ & $9 / 33$ & 27.3 & $5 / 11$ & 45.5 & $2 / 11$ & 1.8 .2 & $2 / 11$ & 18.2 \\
\hline & $0.030-0.059 \%$ & $3 / 18$ & 16.7 & $1 / 6$ & 16.7 & $1 / 6$ & 1.6 .7 & $1 / 6$ & 16.7 \\
\hline & $0.060-0.089 \%$ & $1 / 18$ & 5.6 & $1 / 6$ & 16.7 & $0 / 6$ & 0.0 & $0 / 6$ & 0.0 \\
\hline & $0.090-0.119 \%$ & $2 / 21$ & 9.5 & $2 / 7$ & 28.6 & $0 / 7$ & 0.0 & $0 / 7$ & 0.0 \\
\hline & $0.120-0.149 \%$ & $3 / 42$ & 7.1 & $2 / 14$ & 14.3 & $1 / 14$ & 7.2 & $1 / 14$ & 7.2 \\
\hline & $0.150-0.179 \%$ & $10 / 57$ & 17.5 & $8 / 19$ & 42.1 & $1 / 19$ & 5.3 & $3 / 19$ & 15.8 \\
\hline & $\geq 0.180 \%$ & $5 / 21$ & 23.8 & $5 / 7$ & 71.4 & $0 / 7$ & 0.0 & $1 / 7$ & 14.3 \\
\hline \multirow{7}{*}{$\begin{array}{l}\mathrm{AGE} \\
\geq 30\end{array}$} & $<0.030 \%$ & $10 / 87$ & 11.5 & $7 / 29$ & 24. 1 & $2 / 29$ & 6.9 & $4 / 29$ & 13.8 \\
\hline & $0.030-0.059 \%$ & $7 / 45$ & 15.6 & $5 / 15$ & 33.3 & $2 / 15$ & 13.3 & $4 / 15$ & 26.7 \\
\hline & $0.060-0.089 \%$ & $3 / 30$ & 10.0 & $3 / 10$ & 30.0 & $0 / 10$ & 0.0 & $1 / 10$ & 10.0 \\
\hline & $0.090-0.119 \%$ & $11 / 69$ & 15.9 & $8 / 23$ & 34.8 & $3 / 23$ & 13.1 & $5 / 23$ & 21.7 \\
\hline & $0.120-0.149 \%$ & $34 / 102$ & 33.3 & $20 / 34$ & 58.8 & $10 / 34$ & 2.9 .4 & $14 / 34$ & 41.2 \\
\hline & $0.150-0.179 \%$ & $55 / 114$ & 48.2 & $27 / 38$ & 71.1 & $19 / 38$ & 5.0 .0 & $22 / 38$ & 57.9 \\
\hline & $\geq 0.180 \%$ & $26 / 45$ & 57.8 & $15 / 151$ & 100.0 & $8 / 15$ & 53.3 & $9 / 15$ & 60.0 \\
\hline
\end{tabular}


Table II

Complex Reaction Tester Performance, Group II Response time: 1.8 seconds

Error tolerance: 0

STRATEGY

\begin{tabular}{|c|c|c|c|c|c|c|c|c|c|}
\hline \multirow{2}{*}{$\begin{array}{l}\text { SUBJECT } \\
\text { CATEGORY }\end{array}$} & \multirow{2}{*}{$\begin{array}{l}\text { BAC CLASS } \\
\text { INTER VAL }\end{array}$} & \multicolumn{2}{|c|}{$1 / 1$} & \multicolumn{2}{|c|}{$3 / 3$} & \multicolumn{2}{|c|}{$2 / 3$} & \multicolumn{2}{|c|}{$2 / 2$} \\
\hline & & $\mathrm{N}$ & $\%$ & $\mathrm{~N}$ & $\%$ & $\mathrm{~N}$ & $\%$ & $\mathrm{~N}$ & $\%$ \\
\hline \multirow{7}{*}{$\begin{array}{l}\text { ALL } \\
\text { SUBJECTS }\end{array}$} & $<0.030 \%$ & $4 / 138$ & 2.9 & $3 / 46$ & 6.5 & $1 / 46$ & 2.2 & $3 / 46$ & 6.5 \\
\hline & $0.030-0.059 \%$ & $2 / 96$ & 2.1 & $2 / 32$ & 6.2 & $0 / 32$ & 0.0 & $0 / 32$ & 0.0 \\
\hline & $0.060-0.089 \%$ & $3 / 48$ & 6.2 & $3 / 16$ & 18.7 & $0 / 16$ & 0.0 & $1 / 16$ & 6.2 \\
\hline & $0.090-0.119 \%$ & $9 / 105$ & 8.6 & $8 / 35$ & 22.9 & $1 / 35$ & 2.9 & $3 / 35$ & 8.6 \\
\hline & $0.120-0.149 \%$ & $9 / 105$ & 8.6 & $9 / 35$ & 25.7 & $0 / 35$ & 0.0 & $2 / 35$ & $5 / 7$ \\
\hline & $0.150-0.179 \%$ & $15 / 126$ & 11.9 & $11 / 42$ & 26.2 & $4 / 42$ & 9.5 & $6 / 42$ & 14.3 \\
\hline & $=0.180 \%$ & $12 / 84$ & 14.3 & $11 / 28$ & 39.3 & $1 / 28$ & 3.6 & $5 / 28$ & 17.9 \\
\hline \multirow{7}{*}{ MALES } & $<0.030 \%$ & $3 / 75$ & 4.0 & $2 / 25$ & 8.0 & $1 / 25$ & 4.0 & $2 / 25$ & 8.0 \\
\hline & $0.030-0.059 \%$ & $0 / 48$ & 0.0 & $0 / 16$ & 0.0 & $0 / 16$ & 0.0 & $0 / 16$ & 0.0 \\
\hline & $0.060-0.089 \%$ & $2 / 18$ & 11.1 & $2 / 6$ & 33.3 & $0 / 6$ & 0.0 & $1 / 6$ & 16.7 \\
\hline & $0.090-0.119 \%$ & $5 / 51$ & 9.8 & $4 / 17$ & 23.5 & $1 / 17$ & 5.9 & $2 / 17$ & 11.8 \\
\hline & $0.120-0.149 \%$ & $6 / 54$ & 11.1 & $6 / 18$ & 33.3 & $0 / 18$ & 0.0 & $1 / 18$ & 5.6 \\
\hline & $0.150-0.179 \%$ & $11 / 69$ & 15.9 & $9 / 23$ & 39.1 & $2 / 23$ & 8.7 & $4 / 23$ & 17.4 \\
\hline & $=0.180 \%$ & $8 / 54$ & 14.8 & $7 / 18$ & 38.9 & $1 / 18$ & 5.6 & $5 / 18$ & 27.8 \\
\hline \multirow{7}{*}{ FEMALES } & $<0.030 \%$ & $1 / 63$ & 1.6 & $1 / 21$ & 4.8 & $0 / 21$ & 0.0 & $1 / 21$ & 4.8 \\
\hline & $0.030-0.059 \%$ & $2 / 48$ & 4.2 & $2 / 16$ & 12.5 & $0 / 16$ & 0.0 & $0 / 16$ & 0.0 \\
\hline & $0.060-0.089 \%$ & $1 / 30$ & 3.3 & $1 / 10$ & 10.0 & $0 / 10$ & 0.0 & $0 / 10$ & 0.0 \\
\hline & $0.090-0.119 \%$ & $4 / 54$ & 7.4 & $4 / 18$ & 22.2 & $0 / 18$ & 0.0 & $1 / 18$ & 5.6 \\
\hline & $0.120-0.149 \%$ & $3 / 51$ & 5.9 & $3 / 17$ & 17.6 & $0 / 17$ & 0.0 & $1 / 17$ & 5.9 \\
\hline & $0.150-0.179 \%$ & $4 / 57$ & 7.1 & $2 / 19$ & 10.5 & $2 / 19$ & 10.5 & $2 / 19$ & 10.5 \\
\hline & $\geq 0.180 \%$ & $4 / 30$ & 13.3 & $4 / 10$ & 40.0 & $0 / 10$ & 0.0 & $0 / 10$ & 0.0 \\
\hline \multirow{7}{*}{$\begin{array}{l}\text { AGE } \\
<30\end{array}$} & $<0.030 \%$ & $4 / 90$ & 4.4 & $3 / 30$ & 10.0 & $1 / 30$ & 3.3 & $3 / 30$ & 10.0 \\
\hline & $0.030-0.059 \%$ & $2 / 66$ & 3.1 & $2 / 22$ & 9.1 & $0 / 22$ & 0.0 & $0 / 22$ & 0.0 \\
\hline & $0.060-0.089 \%$ & $2 / 33$ & 6.1 & $2 / 11$ & 18.2 & $0 / 11$ & 0.0 & $1 / 11$ & 9.1 \\
\hline & $0.090-0.119 \%$ & $6 / 78$ & 7.7 & $6 / 26$ & 23.1 & $0 / 26$ & 0.0 & $2 / 26$ & 7.7 \\
\hline & $0.120-0.149 \%$ & $6 / 69$ & 8.7 & $6 / 23$ & 26.1 & $0 / 23$ & 0.0 & $1 / 23$ & 4.3 \\
\hline & $0.150-0.179 \%$ & $11 / 78$ & 14.1 & $8 / 26$ & 30.8 & $3 / 26$ & 11.5 & $4 / 26$ & 15.4 \\
\hline & $\geq 0.180 \%$ & $8 / 54$ & 14.8 & $7 / 18$ & 38.9 & $1 / 18$ & 5.6 & $5 / 18$ & 27.8 \\
\hline \multirow{7}{*}{$\begin{array}{l}\text { AGE } \\
\geq 30\end{array}$} & $<0.030 \%$ & $0 / 48$ & 0.0 & $0 / 16$ & 0.0 & $0 / 16$ & 0.0 & $0 / 16$ & 0.0 \\
\hline & $0.030-0.059 \%$ & $0 / 30$ & 0.0 & $0 / 10$ & 0.0 & $0 / 10$ & 0.0 & $0 / 10$ & 0.0 \\
\hline & $0.060-0.089 \%$ & $1 / 15$ & 6.7 & $1 / 5$ & 20.0 & $0 / 5$ & 0.0 & $0 / 5$ & 0.0 \\
\hline & $0.090-0.119 \%$ & $3 / 27$ & 11.1 & $2 / 9$ & 22.2 & $1 / 9$ & 11.1 & $1 / 9$ & 11.1 \\
\hline & $0.120-0.149 \%$ & $3 / 36$ & 8.3 & $3 / 12$ & 25.0 & $0 / 12$ & 0.0 & $1 / 12$ & 8.3 \\
\hline & $0.150-0.179 \%$ & $4 / 48$ & 8.3 & $3 / 16$ & 18.7 & $1 / 16$ & 6.2 & $2 / 16$ & 12.5 \\
\hline & $=0.180 \%$ & $4 / 30$ & 13.3 & $4 / 10$ & 40.0 & $0 / 10$ & 0.0 & $0 / 10$ & 0.0 \\
\hline
\end{tabular}


Table III

Complex Reaction Tester Performance, Group III Response time: 0.9 seconds

Error tolerence: 0

STRATEGY

\begin{tabular}{|c|c|c|c|c|c|c|c|c|c|}
\hline $\begin{array}{l}\text { SUBJECT } \\
\text { CATEGORY }\end{array}$ & $\begin{array}{l}\text { BAC CLASS } \\
\text { INTERVAL }\end{array}$ & \multicolumn{2}{|c|}{$1 / 1$} & \multicolumn{2}{|c|}{$3 / 3$} & \multicolumn{2}{|c|}{$2: / 3$} & \multicolumn{2}{|c|}{$2 / 2$} \\
\hline \multirow{7}{*}{$\begin{array}{l}\text { ALL } \\
\text { SUBJECTS }\end{array}$} & $<0.030 \%$ & $29 / 177$ & 16.4 & $23 / 59$ & 39.0 & $4 / 59$ & 6.8 & $11 / 59$ & 18.6 \\
\hline & $0.030=0.059 \%$ & $15 / 72$ & 20.8 & $10 / 24$ & 41.7 & $4 / 24$ & 16.7 & $7 / 24$ & 29.2 \\
\hline & $0.060-0.089 \%$ & $10 / 57$ & 17.5 & $8 / 19$ & 42.1 & $2 / 19$ & 10.5 & $5 / 19$ & 26.3 \\
\hline & $0.090-0.119 \%$ & $29 / 108$ & 26.9 & $19 / 36$ & 52.8 & $9 / 36$ & 25.0 & $12 / 36$ & 33.3 \\
\hline & $0.120-0.149 \%$ & $47 / 147$ & 32.0 & $26 / 49$ & 53.1 & $14 / 49$ & 28.6 & $17 / 49$ & 34.7 \\
\hline & $0.150-0.179 \%$ & $69 / 156$ & 44.2 & $35 / 52$ & 67.3 & $23 / 52$ & 44.2 & $27 / 52$ & 51.9 \\
\hline & $\geq 0.180 \%$ & $35 / 63$ & 55.6 & $17 / 21$ & 81.0 & $12 / 21$ & 57.1 & $14 / 21$ & 66.7 \\
\hline \multirow{7}{*}{ MALES } & $<0.030 \%$ & $23 / 108$ & 21.3 & $17 / 36$ & 47.2 & $4 / 36$ & 11.1 & $9 / 36$ & 25.0 \\
\hline & $0.030-0.059 \%$ & $9 / 51$ & 17.6 & $6 / 17$ & 35.3 & $3 / 17$ & 17.6 & 4. 17 & 23.5 \\
\hline & $0.060-0.089 \%$ & $5 / 36$ & 13.9 & $4 / 12$ & 33.3 & $1 / 12$ & 8.3 & $3 / 12$ & 25.0 \\
\hline & $0.090-0.119 \%$ & $20 / 84$ & 23.8 & $13 / 28$ & 46.4 & $6 / 28$ & 21.4 & $8 / 28$ & 28.6 \\
\hline & $0.120-0.149 \%$ & $31 / 102$ & 30.4 & $18 / 34$ & 52.9 & $9 / 34$ & 26.5 & $11 / 34$ & 32.4 \\
\hline & $0.150-0.179 \%$ & $34 / 90$ & 37.8 & $17 / 30$ & 56.7 & $11 / 30$ & 36.7 & $13 / 30$ & 43.3 \\
\hline & $=0.180 \%$ & $13 / 33$ & 39.4 & $8 / 11$ & 72.7 & $4 / 11$ & 36.4 & $5 / 11$ & 45.5 \\
\hline \multirow{7}{*}{ FEMALES } & $=0.030 \%$ & $6 / 69$ & 8. 7 & $6 / 23$ & 26.1 & $0 / 23$ & 0.0 & $2 / 23$ & 8.7 \\
\hline & $0.030-0.059 \%$ & $6 / 21$ & 28.6 & $4 / 7$ & 57.1 & $1 / 7$ & 14.3 & $3 / 7$ & 42.9 \\
\hline & $0.060-0.089 \%$ & $5 / 21$ & 23.8 & $4 / 7$ & 57.1 & $1 / 7$ & 14.3 & $2 / 7$ & 28.6 \\
\hline & $0.090-0.119 \%$ & $9 / 24$ & 37.5 & $6 / 8$ & 75.0 & $3 / 8$ & 37.5 & $4 / 8$ & 50.0 \\
\hline & $0.120-0.149 \%$ & $16 / 45$ & 35.6 & $8 / 15$ & 53.3 & $5 / 15$ & 33.3 & $6 / 15$ & 40.0 \\
\hline & $0.150-0.179 \%$ & $35 / 66$ & 53.1 & $18 / 22$ & 81.8 & $12 / 22$ & 54.5 & $14 / 22$ & 63.6 \\
\hline & $=0.180 \%$ & $22 / 30$ & 73.3 & $9 / 10$ & 90.0 & $8 / 10$ & 80.0 & $9 / 10$ & 90.0 \\
\hline \multirow{7}{*}{$\begin{array}{l}\text { AGE } \\
=30\end{array}$} & $<0.030 \%$ & $9 / 75$ & 12.0 & $2 / 25$ & 36.0 & $0 / 25$ & 0.0 & $4 / 25$ & 16.0 \\
\hline & $0.030-0.059 \%$ & $10 / 42$ & 23.8 & $6 / 14$ & 42.9 & $3 / 14$ & 21.4 & $4 / 14$ & 28.6 \\
\hline & $0.060-0.089 \%$ & $3 / 21$ & 14.3 & $3 / 7$ & 42.9 & $0 / 7$ & 0.0 & $1 / 7$ & 14.3 \\
\hline & $0.090-0.119 \%$ & $12 / 39$ & 30.8 & $7 / 13$ & 52.8 & $4 / 13$ & 30.8 & $6 / 13$ & 46.2 \\
\hline & $0.120-0.149 \%$ & $29 / 87$ & 33.3 & $15 / 29$ & 51.7 & $8 / 29$ & 27.6 & $10 / 29$ & 34.5 \\
\hline & $0.150-0.179 \%$ & $32 / 69$ & 46.4 & $16 / 23$ & 69.6 & $10 / 23$ & 43,5 & $12 / 23$ & 52.2 \\
\hline & $=0.180 \%$ & $15 / 27$ & 55.6 & $7 / 9$ & 77.8 & $6 / 9$ & 66.7 & $6 / 9$ & 66.7 \\
\hline \multirow{7}{*}{$\begin{array}{l}A G E \\
=30\end{array}$} & $<0.030 \%$ & $20 / 102$ & 19.6 & $14 / 34$ & 41.2 & $4 / 34$ & 11.8 & $7 / 34$ & 20.6 \\
\hline & $0.030-0.059 \%$ & $5 / 30$ & 16.7 & $4 / 10$ & 40.0 & $1 / 10$ & 10.0 & $3 / 10$ & 30.0 \\
\hline & $0.060-0.089 \%$ & $7 / 36$ & 19.4 & $5 / 12$ & 41.7 & $2 / 12$ & 16.7 & $4 / 12$ & 33.3 \\
\hline & $0.090-0.119 \%$ & $17 / 69$ & 24.6 & $12 / 23$ & 52,2 & $5 / 23$ & 21.7 & $6 / 23$ & 26.1 \\
\hline & $0.120-0.149 \%$ & $18 / 60$ & 30.0 & $11 / 20$ & 55.0 & $6 / 20$ & 30.0 & $7 / 20$ & 35.0 \\
\hline & $0.150-0.179 \%$ & $37 / 87$ & 42.5 & $19 / 29$ & 65.5 & $14 / 29$ & 48.3 & $15 / 29$ & 51.7 \\
\hline & $=0.180 \%$ & $20 / 36$ & 55.6 & $10 / 12$ & 83.3 & $6 / 12$ & 50.0 & $8 / 12$ & 66.7 \\
\hline
\end{tabular}


Complex Reaction Tester Control Session Performance, Group III Response Time: 0.9 seconds

Error Tolerence: 0

\begin{tabular}{|c|c|c|c|c|c|c|c|c|c|c|}
\hline \multirow{3}{*}{$\begin{array}{l}\text { SUBJECT } \\
\text { CATEGORY }\end{array}$} & \multirow{3}{*}{$\begin{array}{c}\text { TEST } \\
\vdots \\
\end{array}$} & \multirow{3}{*}{$\begin{array}{l}\text { CYCLE } \\
\text { TIME* }\end{array}$} & \multicolumn{8}{|c|}{ STRATEGY } \\
\hline & & & \multicolumn{2}{|c|}{$1 / 1$} & \multicolumn{2}{|c|}{$3 / 3$} & \multicolumn{2}{|c|}{$2 / 3$} & \multicolumn{2}{|c|}{$2 / 2$} \\
\hline & & & $\mathrm{N}$ & $\%$ & $N$ & $\%$ & $\mathrm{~N}$ & $\%$ & $\mathrm{~N}$ & $\%$ \\
\hline \multirow{9}{*}{$\begin{array}{l}\text { ALL } \\
\text { SUBJECTS }\end{array}$} & 1. & $0: 10$ & $8 / 42$ & 19.1 & $6 / 14$ & 42.9 & $1 / 14$ & 7.1 & $3 / 14$ & 21.4 \\
\hline & 2. & $0: 35$ & $7 / 42$ & 16.7 & $5 / 14$ & 35.7 & $2 / 14$ & 14.3 & $3 / 14$ & 21.4 \\
\hline & 3. & $1: 35$ & $2 / 42$ & 4.8 & $2 / 14$ & 14.3 & $0 / 14$ & 0.0 & $0 / 14$ & 0.0 \\
\hline & 4. & $2: 35$ & $5 / 42$ & 11.9 & $4 / 14$ & 28.6 & $1 / 14$ & 7.1 & $3 / 14$ & 21.4 \\
\hline & 5. & $3: 35$ & $5 / 42$ & 11.9 & $3 / 14$ & 21.4 & $1 / 14$ & 7.1 & $2 / 14$ & 14.3 \\
\hline & 6. & $4: 35$ & $9 / 42$ & 21.4 & $5 / 14$ & 35.7 & $3 / 14$ & 21.4 & $4 / 14$ & 28.6 \\
\hline & 7. & 5:00 & $8 / 42$ & 19.1 & $6 / 14$ & 42.9 & $1 / 14$ & 7.1 & $2 / 14$ & 14.3 \\
\hline & 8. & $6: 00$ & $5 / 42$ & 11.9 & $5 / 14$ & 35.7 & $0 / 14$ & 0.0 & $2 / 14$ & 14.3 \\
\hline & 9. & $6: 30$ & $5 / 42$ & 11.9 & $5 / 14$ & 35.7 & $0 / 14$ & 0.0 & $1 / 14$ & 7.1 \\
\hline \multirow{9}{*}{ MALES } & 1. & $0: 10$ & $3 / 24$ & 12.5 & $2 / 8$ & 25.0 & $0 / 8$ & 0.0 & $1 / 8$ & 12.5 \\
\hline & 2. & $0: 35$ & $3 / 24$ & 12.5 & $3 / 8$ & 37.5 & $2 / 8$ & 25.0 & $2 / 8$ & 25.0 \\
\hline & 3. & $1: 35$ & $1 / 24$ & 4.2 & $1 / 8$ & 12.5 & $0 / 8$ & 0.0 & $0 / 8$ & 0.0 \\
\hline & 4. & $2: 35$ & $3 / 24$ & 12.5 & $1 / 8$ & 12.5 & $0 / 8$ & 0.0 & $1 / 8$ & 12.5 \\
\hline & 5. & $3: 35$ & $2 / 24$ & 8.3 & $1 / 8$ & 12.5 & $0 / 8$ & 0.0 & $0 / 8$ & 0.0 \\
\hline & 6. & $4: 35$ & $6 / 24$ & 25.0 & $3 / 8$ & 37.5 & $1 / 8$ & 12.5 & $2 / 8$ & 25.0 \\
\hline & 7. & $5: 00$ & $5 / 24$ & 20.8 & $3 / 8$ & 37.5 & $1 / 8$ & 12.5 & $1 / 8$ & 12.5 \\
\hline & 8. & $6: 00$ & $2 / 24$ & 8.3 & $3 / 8$ & 37.5 & $0 / 8$ & 0.0 & $1 / 8$ & 12.5 \\
\hline & 9. & $6: 30$ & $1 / 24$ & 4.2 & $2 / 8$ & 25.0 & $0 / 8$ & 0.0 & $0 / 8$ & 0.0 \\
\hline \multirow{9}{*}{ FEMALES } & 1. & $0: 10$ & $5 / 18$ & 27.8 & $4 / 6$ & 66.7 & $1 / 6$ & 16.7 & $2 / 6$ & 33.3 \\
\hline & 2. & $0: 35$ & $4 / 18$ & 22.2 & $2 / 6$ & 33.3 & $0 / 6$ & 0.0 & $1 / 6$ & 16.7 \\
\hline & 3. & $1: 35$ & $1 / 18$ & 5.6 & $1 / 6$ & 16.7 & $0 / 6$ & 0.0 & $0 / 6$ & 0.0 \\
\hline & 4. & $2: 35$ & $2 / 18$ & 11.1 & $3 / 6$ & 50.0 & $1 / 6$ & 16.7 & $2 / 6$ & 33.3 \\
\hline & 5. & $3: 35$ & $3 / 18$ & 16.7 & $2 / 6$ & 33.3 & $1 / 6$ & 16.7 & $2 / 6$ & 33.3 \\
\hline & 6. & $4: 35$ & $3 / 18$ & 16.7 & $2 / 6$ & 33.3 & $2 / 6$ & 33.3 & $2 / 6$ & 33.3 \\
\hline & 7. & $5: 00$ & $3 / 18$ & 16.7 & $3 / 6$ & 50.0 & $0 / 6$ & 0.0 & $1 / 6$ & 16.7 \\
\hline & 8. & $6: 00$ & $3 / 18$ & 16.7 & $2 / 6$ & 33.3 & $0 / 6$ & 0.0 & $1 / 6$ & 16.7 \\
\hline & 9. & $6: 30$ & $4 / 18$ & 22.2 & $3 / 6$ & 50.0 & $0 / 6$ & 0.0 & $1 / 6$ & 16.7 \\
\hline \multirow{9}{*}{$A G E<30$} & 1. & $0: 10$ & $5 / 18$ & 27.8 & $3 / 6$ & 50.0 & $1 / 6$ & 16.7 & $2 / 6$ & 33.3 \\
\hline & 2. & $0: 35$ & $4 / 18$ & 22.2 & $3 / 6$ & 50.0 & $1 / 6$ & 16.7 & $2 / 6$ & 33.3 \\
\hline & 3. & $1: 35$ & $1 / 18$ & 5.6 & $1 / 6$ & 16.7 & $0 / 6$ & 0.0 & $0 / 6$ & 0.0 \\
\hline & 4. & $2: 35$ & $2 / 18$ & 11.1 & $1 / 6$ & 16.7 & $1 / 6$ & 16.7 & $1 / 6$ & 16.7 \\
\hline & 5. & $3: 35$ & $3 / 18$ & 16.7 & $1 / 6$ & 16.7 & $1 / 6$ & 16.7 & $1 / 6$ & 16.7 \\
\hline & 6. & $4: 35$ & $3 / 18$ & 16.7 & $1 / 6$ & 16.7 & $1 / 6$ & 16.7 & $1 / 6$ & 16.7 \\
\hline & 7. & $5: 00$ & $3 / 18$ & 16.7 & $3 / 6$ & 50.0 & $0 / 6$ & 0.0 & $0 / 6$ & 0.0 \\
\hline & 8. & $6: 00$ & $3 / 18$ & 16.7 & $3 / 6$ & 50.0 & $0 / 6$ & 0.0 & $1 / 6$ & 16.7 \\
\hline & 9. & $6: 30$ & $4 / 18$ & 22.2 & $4 / 6$ & 66.7 & $0 / 6$ & 0.0 & $1 / 6$ & 16.7 \\
\hline \multirow{9}{*}{$A G E \geq 30$} & 1. & $0: 10$ & $3 / 24$ & 12.5 & $3 / 8$ & 37.5 & $0 / 8$ & 0.0 & $1 / 8$ & 12.5 \\
\hline & 2. & $0: 35$ & $3 / 24$ & 12.5 & $2 / 8$ & 25.0 & $1 / 8$ & 12.5 & $1 / 8$ & 12.5 \\
\hline & 3. & $1: 35$ & $1 / 24$ & 4.2 & $1 / 8$ & 12.5 & $0 / 8$ & 0.0 & $0 / 8$ & 0.0 \\
\hline & 4. & $2: 35$ & $3 / 24$ & 12.5 & $3 / 8$ & 37.5 & $0 / 8$ & 0.0 & $2 / 8$ & 25.0 \\
\hline & 5. & $3: 35$ & $2 / 24$ & 8.3 & $2 / 8$ & 25.0 & $0 / 8$ & 0.0 & $1 / 8$ & 12.5 \\
\hline & 6. & $4: 35$ & $6 / 24$ & 25.0 & $4 / 8$ & 50.0 & $2 / 8$ & 25.0 & $3 / 8$ & 37.5 \\
\hline & 7. & 5:00 & $5 / 24$ & 20.8 & $3 / 8$ & 37.5 & $1 / 8$ & 12.5 & $2 / 8$ & 25.0 \\
\hline & 8. & $6: 00$ & $2 / 24$ & 8.3 & $2 / 8$ & 25.0 & $0 / 8$ & 0.0 & $1 / 8$ & 12.5 \\
\hline & 9. & $6: 30$ & $1 / 24$ & 4.2 & $1 / 8$ & 12.5 & $0 / 8$ & 0.0 & $0 / 8$ & 0.0 \\
\hline
\end{tabular}


Table V

Complex Reaction Tester Performance, Group IIIC

Response time: 0.9 seconds

Error tolerance: 0

STRATEGY

\begin{tabular}{|c|c|c|c|c|c|c|c|c|c|}
\hline \multirow{2}{*}{$\begin{array}{l}\text { SUBJECT } \\
\text { CATEGORY }\end{array}$} & \multirow{2}{*}{$\begin{array}{l}\text { BAC CLASS } \\
\text { INTER VAL }\end{array}$} & \multicolumn{2}{|c|}{$1 / 1$} & \multicolumn{2}{|c|}{$3 / 3$} & \multicolumn{2}{|c|}{$2 / 3$} & \multicolumn{2}{|c|}{$2 / 2$} \\
\hline & & $\mathrm{N}$ & $\%$ & $\mathrm{~N}$ & $\%$ & $\mathrm{~N}$ & $\%$ & $\mathrm{~N}$ & $\%$ \\
\hline \multirow{7}{*}{$\begin{array}{l}\text { ALL } \\
\text { SUBJECTS }\end{array}$} & $=0.030 \%$ & $6 / 33$ & 18.2 & $3 / 11$ & 27.3 & $3 / 11$ & 27.3 & $3 / 11$ & 27,3 \\
\hline & $0.030-0.059 \%$ & $2 / 12$ & 16.7 & $2 / 4$ & 50.0 & $0 / 4$ & 0.0 & $1 / 4$ & 25.0 \\
\hline & $0.060-0.089 \%$ & $0 / 9$ & 0.0 & $0 / 3$ & 0.0 & $0 / 3$ & 0.0 & $0 / 3$ & 0.0 \\
\hline & $0.090-0.119 \%$ & $3 / 15$ & 20.00 & $3 / 5$ & 60.0 & $0 / 5$ & 0.0 & $0 / 5$ & 0.0 \\
\hline & $0.120-0.149 \%$ & $3 / 24$ & 12.5 & $3 / 8$ & 37.5 & $0 / 8$ & 0.0 & $2 / 8$ & 25.0 \\
\hline & $0.150-0.179 \%$ & $7 / 33$ & 21.2 & $6 / 11$ & 54.5 & $1 / 11$ & 9.1 & $4 / 11$ & 36.4 \\
\hline & $\geq 0.180 \%$ & $8 / 18$ & 44.4 & $5 / 6$ & 83.3 & $3 / 6$ & 50.0 & $4 / 6$ & 66.7 \\
\hline \multirow{7}{*}{ MALES } & $<0.030 \%$ & $4 / 9$ & 44.4 & $2 / 3$ & 66.7 & $2 / 3$ & 66.7 & $2 / 3$ & 66.7 \\
\hline & $0.030-0.059 \%$ & $1 / 3$ & 33.0 & $1 / 1$ & 100,0 & $0 / 1$ & 0.0 & $0 / 1$ & 0.0 \\
\hline & $0.060-0.089 \%$ & $0 / 3$ & 0.0 & $0 / 1$ & 0.0 & $0 / 1$ & 0.0 & $0 / 1$ & 0.0 \\
\hline & $0.090-0.119 \%$ & $1 / 6$ & 16.7 & $1 / 2$ & 50.0 & $0 / 2$ & $\underline{0.0}$ & $0 / 2$ & 0.0 \\
\hline & $0.120-0.149 \%$ & & & & - & & & & - \\
\hline & $0.150-0.179 \%$ & $4 / 12$ & 33.3 & $3 / 4$ & 75.0 & $1 / 4$ & 25.0 & $2 / 4$ & 50.0 \\
\hline & $=0.180 \%$ & $7 / 15$ & 46.7 & $4 / 5$ & 80.0 & $3 / 5$ & 60.0 & $3 / 5$ & 60.0 \\
\hline \multirow{7}{*}{ FEMALES } & $<0.030 \%$ & $2 / 24$ & 8.3 & $1 / 8$ & 12.5 & $1 / 8$ & 12.5 & $1 / 8$ & 12.5 \\
\hline & $0.030-0.059 \%$ & $1 / 9$ & 11.1 & $1 / 3$ & 33.3 & $0 / 3$ & 0.0 & $1 / 3$ & 33.3 \\
\hline & $0.060-0.089 \%$ & $0 / 6$ & 0.0 & $0 / 2$ & 0.0 & $0 / 2$ & 0.0 & $0 / 2$ & 0.0 \\
\hline & $0.090-0.119 \%$ & $2 / 9$ & 22.2 & $2 / 3$ & 66.7 & $0 / 3$ & 0.0 & $0 / 3$ & 0.0 \\
\hline & $0.120-0.149 \%$ & $3 / 24$ & 12.5 & $3 / 8$ & 37.5 & $0 / 8$ & 0.0 & $2 / 8$ & 25.0 \\
\hline & $0.150-0.179 \%$ & $3 / 21$ & 14.3 & $3 / 7$ & 42.9 & $0 / 7$ & $\underline{0.0}$ & $2 / 7$ & 28.6 \\
\hline & $\geq 0.180 \%$ & $1 / 3$ & 33.3 & $1 / 1$ & 100,0 & $0 / 1$ & 0.0 & $1 / 1$ & 100.0 \\
\hline \multirow{7}{*}{$\begin{array}{l}\text { AGE } \\
=30\end{array}$} & $<0.030 \%$ & $0 / 15$ & 0.0 & $0 / 5$ & 0.0 & $0 / 5$ & 0.0 & $0 / 5$ & 0.0 \\
\hline & $0.030-0.059 \%$ & $0 / 3$ & 0.0 & $0 / 1$ & 0.0 & $0 / 1$ & 0.0 & $0 / 1$ & 0.0 \\
\hline & $0.060-0.089 \%$ & $0 / 3$ & 0.0 & $0 / 1$ & 0.0 & $0 / 1$ & 0.0 & $0 / 1$ & 0.0 \\
\hline & $0.090-0.119 \%$ & $0 / 3$ & 0.0 & $0 / 1$ & 0.0 & $0 / 1$ & 0.0 & $0 / 1$ & 0.0 \\
\hline & $0.120-0.149 \%$ & $1 / 12$ & 8.3 & $1 / 4$ & 25.0 & $0 / 4$ & 0.0 & $0 / 4$ & 0.0 \\
\hline & $0.150-0.179 \%$ & $1 / 9$ & 1.1 & $1 / 3$ & 33.3 & $0 / 3$ & 0.0 & $1 / 3$ & 33.3 \\
\hline & $=0.180 \%$ & $1 / 3$ & 33.3 & $1 / 1$ & 100.0 & $0 / 1$ & 0.0 & $1 / 1$ & 100.0 \\
\hline \multirow{7}{*}{$\begin{array}{l}\text { AGE } \\
=30\end{array}$} & $=0.030 \%$ & $6 / 18$ & 33.3 & $3 / 6$ & 50.0 & $3 / 6$ & 50.0 & $3 / 6$ & 50.0 \\
\hline & $0.030-0.059 \%$ & $2 / 9$ & 22.2 & $2 / 3$ & 66.7 & $0 / 3$ & 0.0 & $1 / 3$ & 33.3 \\
\hline & $0.060-0.089 \%$ & $0 / 6$ & 0.0 & $0 / 2$ & 0.0 & $0 / 2$ & 0.0 & $0 / 2$ & 0.0 \\
\hline & $0.090-0.119 \%$ & $3 / 12$ & 25.0 & $3 / 4$ & 75.0 & $0 / 4$ & 0.0 & $0 / 4$ & 0.0 \\
\hline & $0.120-0.149 \%$ & $2 / 12$ & 16.7 & $2 / 4$ & 50.0 & $0 / 4$ & 0.0 & $2 / 4$ & 50.0 \\
\hline & $0.150-0.179 \%$ & $6 / 24$ & 25.0 & $5 / 8$ & 62.5 & $1 / 8$ & 12.5 & $3 / 8$ & 37.5 \\
\hline & $=0.180 \%$ & $7 / 15$ & 46.7 & $4 / 5$ & 80.0 & $3 / 5$ & 60.0 & $3 / 5$ & 60.0 \\
\hline
\end{tabular}


Table VI

Distribution of Complex Reaction Tester Failed Trials by BAC

Flash Location*

\begin{tabular}{|c|c|c|c|c|c|c|c|c|c|}
\hline $\mathrm{BAC}$ & $\mathrm{TL}$ & B L & TR & BR & $\begin{array}{l}\text { Top Row } \\
\text { TL+TR }\end{array}$ & $\begin{array}{c}\text { Bottom } \\
\text { Row } \\
\text { BL+BR }\end{array}$ & $\begin{array}{l}\text { Left Hand } \\
\mathrm{TL}+\mathrm{BR}\end{array}$ & $\begin{array}{c}\text { Right Hand } \\
\mathrm{TR}+\mathrm{BL} \\
\end{array}$ & Totals ${ }^{* * *}$ \\
\hline$<0.030$ & 4 & 7 & 6 & 11 & 10 & 18 & 15 & 13 & 28 \\
\hline $.030-.059$ & 2 & 2 & 4 & 5 & 6 & 7 & 7 & 6 & 13 \\
\hline $.060-.089$ & 3 & 3 & 1 & 2 & 4 & 5 & 5 & 4 & 9 \\
\hline $.090-.119$ & 6 & 4 & 8 & 8 & 14 & 12 & 14 & 12 & 26 \\
\hline $.120-.149$ & 8 & 7 & 9 & 13 & 17 & 20 & 21 & 16 & 37 \\
\hline $.150-.179$ & 18 & 16 & 11 & 11 & 29 & 27 & 29 & 27 & 56 \\
\hline$\geq .180$ & 10 & 8 & 7 & 5 & 17 & 13 & 15 & 15 & 30 \\
\hline Totals & 51 & 47 & 46 & 55 & 97 & 102 & 106 & 93 & \\
\hline
\end{tabular}

$\stackrel{1}{\omega}$

Flash Number

\begin{tabular}{|c|c|c|c|c|c|c|c|c|c|c|c|c|c|}
\hline $\mathrm{BAC}$ & 1 & 2 & 3 & 4 & 5 & 6 & 7 & 8 & $\leq 4$ & $>4$ & $\leq 6$ & $>6$ & Totals \\
\hline$<0.030$ & 5 & 1 & 6 & 6 & 4 & 2 & 2 & 5 & 18 & 13 & 24 & 7 & 31 \\
\hline $.030-.059$ & 0 & 0 & 3 & 2 & 2 & 2 & 3 & 1 & 5 & 8 & 9 & 4 & 13 \\
\hline $.060-.089$ & 2 & 4 & 2 & 0 & 1 & 0 & 1 & 0 & 8 & 2 & 9 & 1 & 10 \\
\hline $.090-.119$ & 5 & 2 & 7 & 4 & 1 & 2 & 4 & 4 & 18 & 11 & 21 & 8 & 29 \\
\hline $.120-.149$ & 13 & 4 & 2 & 7 & 9 & 5 & 5 & 2 & 26 & 21 & 40 & 7 & 47 \\
\hline $.150-.179$ & 14 & 7 & 11 & 13 & 8 & 6 & 5 & 5 & 45 & 24 & 59 & 10 & 69 \\
\hline$\geq .180$ & 6 & 7 & 5 & 4 & 3 & 1 & 5 & 4 & 22 & 13 & 26 & 9 & 35 \\
\hline Totals & 45 & 25 & 36 & 36 & 28 & 18 & 25 & 21 & 142 & 92 & 188 & 46 & \\
\hline
\end{tabular}

**

\footnotetext{
$\mathrm{TL}=$ Top left, BR = Bottom right, etc.

*** Note: Recording of flash number commenced on session number 18, while recording of location began on session number 19 . Hence, totals do not agree.
} 
Table VII

Distribution of Complex Reaction Tester Failed Trials - Control Sessions

Flash Location

\begin{tabular}{|c|c|c|c|c|c|c|c|c|c|c|}
\hline \multicolumn{2}{|c|}{ Cycle } & T L & B L & TR & BR & $\begin{array}{l}\text { Top Row } \\
\text { TL+TR }\end{array}$ & $\begin{array}{l}\text { Bottom } \\
\text { Row } \\
\text { BL + BR }\end{array}$ & $\begin{array}{l}\text { Left Hand } \\
T L+B R\end{array}$ & $\begin{array}{c}\text { Right Hand } \\
\text { TR + BL }\end{array}$ & Totals \\
\hline 1 & $0: 10$ & 4 & 2 & 1 & 1 & 5 & 3 & 5 & 3 & 8 \\
\hline 2 & $0: 35$ & 3 & 1 & 0 & 3 & 3 & 4 & 6 & 1 & 7 \\
\hline 3 & $1: 35$ & 1 & 1 & 0 & 0 & 1 & 1 & 1 & 1 & 2 \\
\hline 4 & $2: 35$ & 3 & 0 & 1 & 2 & 4 & 2 & 5 & 1 & 6 \\
\hline 5 & $3: 35$ & 2 & 0 & 0 & 2 & 2 & 2 & 4 & 0 & 4 \\
\hline 6 & $4: 35$ & 4 & 2 & 2 & 1 & 6 & 3 & 5 & 4 & 9 \\
\hline 7 & $5: 00$ & 4 & 3 & 0 & 1 & 4 & 4 & 5 & 3 & 8 \\
\hline 8 & $6: 00$ & 0 & 2 & 1 & 2 & 1 & 4 & 2 & 3 & 5 \\
\hline 9 & $6: 30$ & 1 & 1 & 0 & 3 & 1 & 4 & 4 & 1 & 5 \\
\hline \multicolumn{2}{|c|}{ Totals } & 22 & 12 & 5 & 15 & 27 & 27 & 37 & 17 & \\
\hline
\end{tabular}

Flash Number

\begin{tabular}{|c|c|c|c|c|c|c|c|c|c|c|c|c|c|c|}
\hline \multicolumn{2}{|c|}{ Cycle } & \multirow[b]{2}{*}{1} & \multirow[b]{2}{*}{2} & \multirow[b]{2}{*}{3} & \multirow[b]{2}{*}{4} & \multirow[b]{2}{*}{5} & \multirow[b]{2}{*}{6} & \multirow[b]{2}{*}{7} & \multirow[b]{2}{*}{8} & \multirow[b]{2}{*}{$\geq 4$} & \multirow[b]{2}{*}{$>4$} & \multirow[b]{2}{*}{$\leq 6$} & \multirow[b]{2}{*}{$\geq 6$} & \multirow[b]{2}{*}{ Totals } \\
\hline No. & Time & & & & & & & & & & & & & \\
\hline 1 & $0: 10$ & 2 & 0 & 0 & 2 & 1 & 0 & 2 & 1 & 4 & 4 & 5 & 3 & 8 \\
\hline 2 & $0: 35$ & 1 & 2 & 1 & 0 & 1 & 1 & 1 & 0 & 4 & 3 & 6 & 1 & 7 \\
\hline 3 & $1: 35$ & 0 & 0 & 0 & 0 & 1 & 0 & 1 & 0 & 0 & 2 & 1 & 1 & 2 \\
\hline 4 & $2: 35$ & 1 & 1 & 2 & 1 & 0 & 0 & 1 & 0 & 5 & 1 & 5 & 1 & 6 \\
\hline 5 & $3: 35$ & 0 & 0 & 0 & 2 & 0 & 0 & 1 & 1 & 2 & 2 & 2 & 2 & 4 \\
\hline 6 & $4: 35$ & 1 & 2 & 3 & 0 & 0 & 1 & 1 & 1 & 6 & 3 & 7 & 2 & 9 \\
\hline 7 & $5: 00$ & 3 & 1 & 0 & 0 & 2 & 0 & 0 & 2 & 4 & 4 & 6 & 2 & 8 \\
\hline 8 & $6: 00$ & 0 & 0 & 0 & 3 & 0 & 1 & 0 & 1 & 3 & 2 & 4 & 1 & 5 \\
\hline 9 & $6: 30$ & 2 & 1 & 0 & 0 & 1 & 0 & 0 & 1 & 3 & 2 & 4 & 1 & 5 \\
\hline \multicolumn{2}{|c|}{ Totals } & 10 & 7 & 6 & 8 & 6 & 3 & 7 & 7 & 31 & 23 & 40 & 14 & \\
\hline
\end{tabular}




\section{PHYSTESTER}

The Phystester, developed by General Motors, employs a divided attention task. The device consists of two major elements, a handset containing a "touch tone"-type keyboard and display, and a foot pedal. To initiate a trial, the Subject must enter a five-digit code* number on the keyboard. If this is done correctly, a five-digit "random" number is displayed for 1.5 seconds, during which time the Subject must memorize it. Once the number disappears, the Subject begins to enter it via the keyboard; at some point during this process a visual stimulus ("BRAKE" light) appears on the display, signalling required action of the foot pedal. The Subject must promptly depress the pedal while continuing to enter the number.

Phystester units examined by Dunlap and Associates, Inc. had response time requirements ranging from 3.0 seconds to 3.6 seconds for the number insertion portion of the task. Response time required for foot pedal activation was unstated, but apparently was relatively short.

"PASS" or "FAIL" of a trial was displayed by illumination of indicators labelled "START" and "SET", respectively, which were located on the handset.

Discussions of Phystester training, testing, and analysis are presented below.

\section{A. Training Procedures}

Training on the Phystester for Groups I and II provided up to 8 blocks of 25 trials on each of 3 sessions. The response time of the device used was 3.6 seconds. Subjects were eligible each session for a $\$ 5$ reward by passing 23 or more trials of a block. Upon achieving criterion, training for the session was discontinued, with eligibility for reward renewed on succeeding sessions. Six of the 10 Subjects in Group I achieved criterion on the first session, 8 on the second, and 9 on the third. One Subject (No. 105) did not succeed in reaching criterion in the three session training period of 600 trials although the percentage of passes increased steadily from $47 \%$ to $69 \%$ to $80 \%$. The mean numbers of trials per Subject over the three sessions were 100.5, 94. 1, and 55. 0 respectively per session with an overall mean of 249.6 trials per Subject.

"This code apparently is intended primarily as an anti-theft feature; during this program, incorrect entry of the code was not construed as a failure of the trial. 
Of the 12 Subjects in Group II, 11 reached training criterion on the first session and all 12 did so on the second and third. The mean numbers of trials per Subject were $68.8,37.5$, and 37.5 on the three sessions for an overall mean of 143.8 trials per Subject. Since the equipment and training procedures were identical for both groups, the most reasonable explanation for this difference is that the individuals of Group II were, on the average, more competent with equipment of this type than were those of Group I. The mean age of Group II is also somewhat lower- -34.2 years as compared with 38.6 years. It should be noted that the Group I Subjects also trained on the Nartron device, whose keyboard differs radically from the Phystester's. Several Subjects commented specifically on the element of confusion this conflict created.

Group III training followed a substantially different paradigm. Testing of Groups I and II had suggested that the task was too easy to provide good discrimination of the influence of alcohol. Thus, it was determined that a more stringent response time criterion would be required for Group III. Four separate Phystester units were made available for testing, each with a different response time. The values were 3.6 seconds (the same as that used by Groups I and II), 3.4 seconds, 3.2 seconds, and 3.0 seconds. * As these units were originally supplied, they were battery powered. (The unit used by Groups I and II had received power from a separate 12 vdc suppl.y.). After it was determined that battery life was substantially less than one day under the trai ning schedule, the units were adapted to accept 6 vdc from a separate power supply. A difficulty was experienced involving faulty display modules on several of the units, especially on the center digit of the 3.2 second response time unit. This required certain alternations in the planned training schedule when it was determined that the faulty display was contributory to a significant failure rate for certain Subjects.

The planned training schedule for Group III included up to 16 blocks of 12 trials for each of the three training sessions. Each Subject started with the 3. 6 second unit and worked toward an intermediate training criterion of 10 passes in a block for a reward of $\$ 1.00$. When this criterion was achieved, he then worked toward intermediate criterion on the 3.4 second unit. Similarly, he progressed toward the 3.2 second and finally the 3.0 secorid unit. This progression was designed to cease either:

- Upon attainment of intermediate criterion on the 3.0 second unit, or When the Subject completed 16 blocks on a particular unit without attaining intermediate criterion.

An early developmental model in which response time could be discretely varied by 0.1 second intervals had been rejected due to extreme susceptibility to static 'electricity. It should be noted that this unit had a metal case for the hand-set. The other Phystester units used all were housed in plastic cases and exhibited no extraordinary sensitivity to static. 
In the former case, the Subject was to be assigned the 3.0 second instrument for the remainder of training and all testing. In the latter case, the Subject was to be assigned the unit on which intermediate criterion most recently had been achieved. Thus, the intent was to determine the appropriate response time testing criterion on an individual basis. All Subjects, however, ultimately progressed to and achieved intermediate criterion on the 3.0 second device. Having done so, they worked toward final training criterion of 23 passes out of 24 trials on two consecutive blocks for a reward of $\$ 5.00$. Training ceased for the session upon achieving this final criterion, but eligibility for this reward was renewed on succeeding training sessions.

All 15 Subjects achieved intermediate criterion on the 3.6 and 3.4 second units on their first training session. The mean numbers of trials to achieve criterion were 40.0 on the 3.6 second unit and 24.8 on the 3.4 second unit. Thirteen of the 15 Subjects achieved intermediate criterion on the 3.2 second unit with a mean of 22.2 trials each. The other two Subjects took 60 and 48 trials respectively without reaching criterion. Twelve of the 15 Subjects were able to strive for intermediate criterion on the 3.0 second unit on their first training session. All were successful and 5 also achieved final criterion. The mean number of trials per Subject across all units was 136.0 on the first session.

On the second session, one of the two Subjects who had not reached intermediate criterion on the 3.2 second unit made an additional 144 trials and still had not done so. Since the faulty center digit of this unit seemed to contribute to this problem, he was instructed to try the 3.0 second unit. On this he achieved intermediate, but not final criterion. For the same reason, the other Subject was moved immediately to the 3.0 unit and achieved both intermediate and final criteria in 48 trials. One Subject who had reached final criterion on his first session failed to repeat within 144 trials on the second. All of the other 12 Subjects achieved final criterion. The mean number of trials for those achieving final criterion was 37.8. The mean number of trials per Subject across both units used was 55.2 on the second training session.

On the third training session, all Subjects attained final criterion on the 3.0 second unit. The mean number of trials per Subject was 36.8 , making an overall mean of 228.0 trials across all machines and all three training sessions. Individual totals ranged from the theoretical minimum of 120 to a high of 408 trials. Similar ranges for Groups I and II were 125-600, and 75-250. The theoretical minimum for both these Groups was 75 trials (achieved twice in Group II). 


\section{B. Testing Procedures}

Testing on the Phystester was consistent for all three groups and was composed of one block of three trials in each testing cycle. The Escort recorded each trial as "pass" or "fail." No penalty was registered for missing the entry code as this was not considered part of the test any more than was turning on the power. (Subjects under the influence of alcohol would occasionally forget to turn the unit on and would frequently miss the code.) For Groups I and II, the reward was $\$ .50$ per pass. For Group III the reward was $\$ 0.25$ per pass with a $100 \%$ bonus if all trials in the block were passed.

The three Subjects participating in the special Quickey Experiment (discussed in Section IV) also tested on the 3.0 second Phystester unit. All had previously trained and tested on the 3,6 second device. They were provided no formal retraining prior to testing, but were simply permitted two or three familiarization trials at the beginning of their first test session.

\section{Analyses and Results}

Phystester performance was examined under the following strategies:

$$
\begin{aligned}
& \text { "one-out-of-one" } \\
& \text { "three-out-of-three" } \\
& \text { "two-out-of-three" } \\
& \text { "two-out-of-two" }
\end{aligned}
$$

Tables VIII, IX, and $X$ exhibit the results of these analysies for Groups I, II, and III, respectively; Table XI shows control session performance demonstrated by the Group III Subjects; Table XII shows results obtained from the three Subjects participating in the special Quickey experiment (Group IIIC).

While markedly different performance is noted among the three groups, all data indicate that strategies $1 / 1$ and $3 / 3$ would induce fairly high failure rates at negligible BAC (ranging from roughly 5\%-35\%); the feasibility of such approaches thus appears doubtful. Strategies of $2 / 3$ and $2 / 2$ appear to offer more promise; their performances are exhibited graphically in Figures 4 and 5.

It can be seen readily that the 3.0 second device (Group III) would reject an appreciably higher proportion of drivers at moderate to high BAC than would the 3. 6 second unit (Groups I and II), as would be expected in view of the increased degree of difficulty. Of perhaps equal importance is the fact that it also exhibits 
a lower rejection rate at very low $B A C$. One plausible explanation for this latter phenomenon may be found in the substantially increased amount of training provided to the Group III Subjects, coupled with the more stringent requirement for testing reward. In any event, the data indicate that the 3.0 second device with a $2 / 2$ or $2 / 3$ strategy merits consideration for future applications.

Analyses of variance indicated significant differences in performance be tween Groups I and III and between Groups II and III under the $2 / 3$ strategy ( $p<.05$ in both cases); no significant difference was noted between Groups 1 and II $(p>20)$. However, there is a striking difference between Groups I and II at high BAC $(\geq 0,180 \%)$. These Subjects experienced exactly the same circumstances of Phystester training and testing; nevertheless, Group II evidenced a much lower rejection rate at this high level under all strategies. Comparison of the two sets of data by age and sex shows that the difference is most pronounced among males and younger Subjects. The most reasonable explanation for this anomaly is that, while high BAC data from Group I were obtained nearly uniformly from all 10 Subjects, two of the 12 Subjects of Group II* jointly provided one-half of all data above 0.180\% BAC. Both Subjects were young males who seemed especially competent with this instrument.

Analyses of variance of $B A C$ versus sex and $B A C$ versus age were applied to performance data corresponding to the $2 / 3$ strategy. Results (listed below) indicate no significant differences with respect to sex and age $(p>10)$.

\begin{tabular}{|c|c|c|c|c|c|}
\hline & \multicolumn{2}{|c|}{ BAC vs. SEX } & \multicolumn{2}{|c|}{$B A C$ vs. $A G E$} \\
\hline & & $B A C$ & SEX & $\mathrm{BAC}$ & $\mathrm{AGE}$ \\
\hline$\underset{I}{\text { Group }}$ & $\begin{array}{l}\text { F } \\
\text { Significance }\end{array}$ & $\begin{array}{c}5.18 \\
.05>p>.01\end{array}$ & $\begin{array}{l}.003 \\
\text { N. S. }\end{array}$ & $\begin{array}{c}5.78 \\
.05=p>.01\end{array}$ & $\begin{array}{c}.013 \\
\text { N. S. }\end{array}$ \\
\hline $\begin{array}{l}\text { Group } \\
\text { II }\end{array}$ & $\begin{array}{l}E \\
\text { Significance }\end{array}$ & $\begin{array}{c}1.09 \\
p>.20\end{array}$ & $\begin{array}{l}0.15 \\
\text { N.S. }\end{array}$ & $\begin{array}{c}1.71 \\
\mathrm{p}=.20\end{array}$ & $\begin{array}{c}2.75 \\
.20=p=.10\end{array}$ \\
\hline $\begin{array}{l}\text { Group } \\
\text { III }\end{array}$ & $\begin{array}{l}\mathrm{F} \\
\text { Significance }\end{array}$ & $\begin{array}{c}4.83 \\
.05>p=.01\end{array}$ & $\begin{array}{c}3.58 \\
.20>p>.10\end{array}$ & $\begin{array}{c}7.01 \\
.05>p>.01\end{array}$ & $\begin{array}{l}1.33 \\
\mathrm{p}=.20\end{array}$ \\
\hline
\end{tabular}

Phystester control session data show the proportion of trials failed peaking at the beginning and end of the test session, which may indicate that both anxiety and fatigue had some influence on test results. These data are compared with

Subjects 114 and 120. 
the Group III drinking sessions results in Figure 6, which also depicts the mean $\mathrm{BAC}$ of each drinking session cycle. Analysis of variance on these data indicates that the main effect for Treatment (Control vs. Drinking) is significant ( $F=13.69$, $p<.01$ ) while that for Cycle is not. It is apparent from the curves in Figure 6 that there is a substantial interaction (treated as the "error" term in this analysis) which tends to mask any cycle effects which may exist and which is not itself testable. 


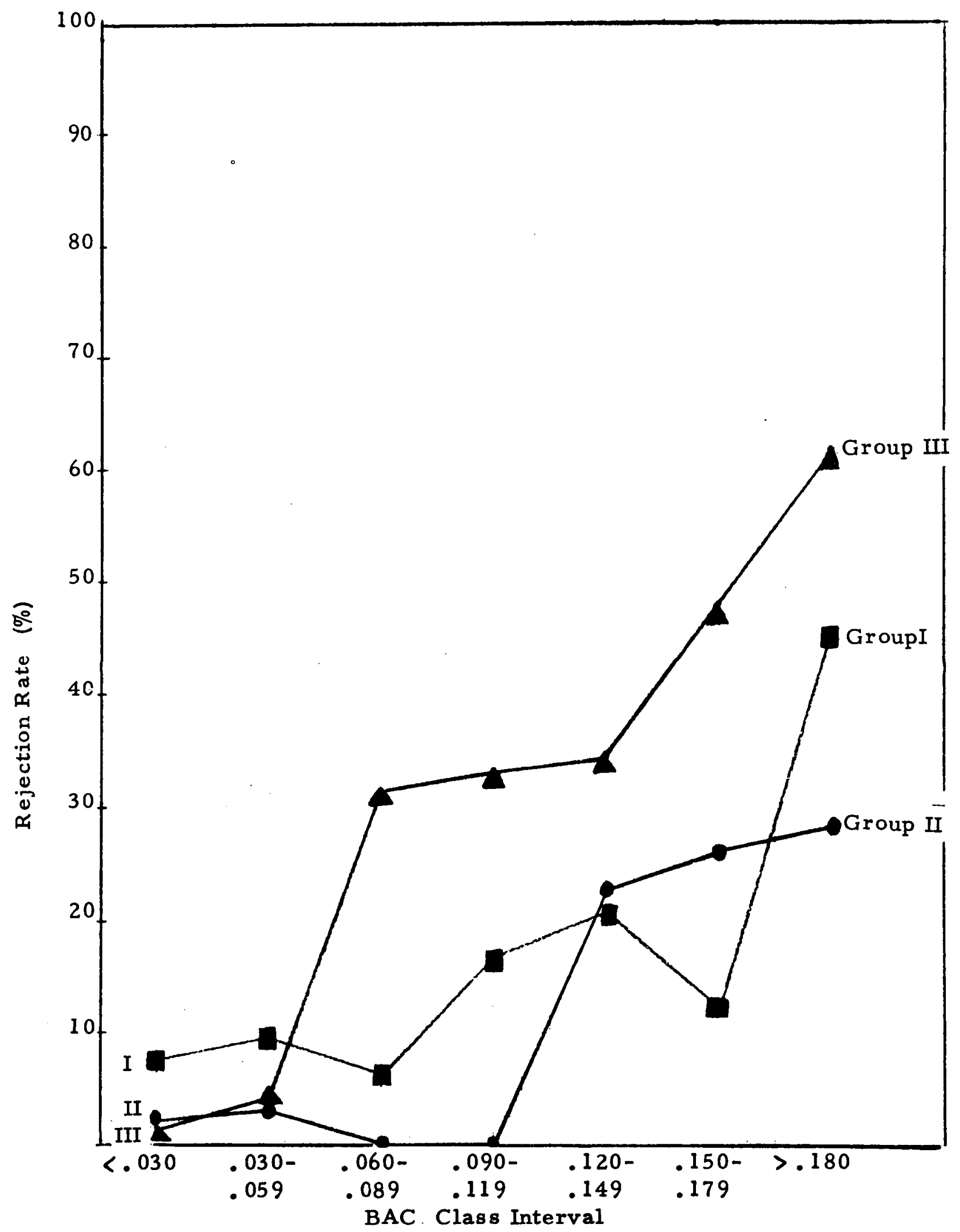

Figure 4. Phystester Performance - $2 / 3$ Strategy 


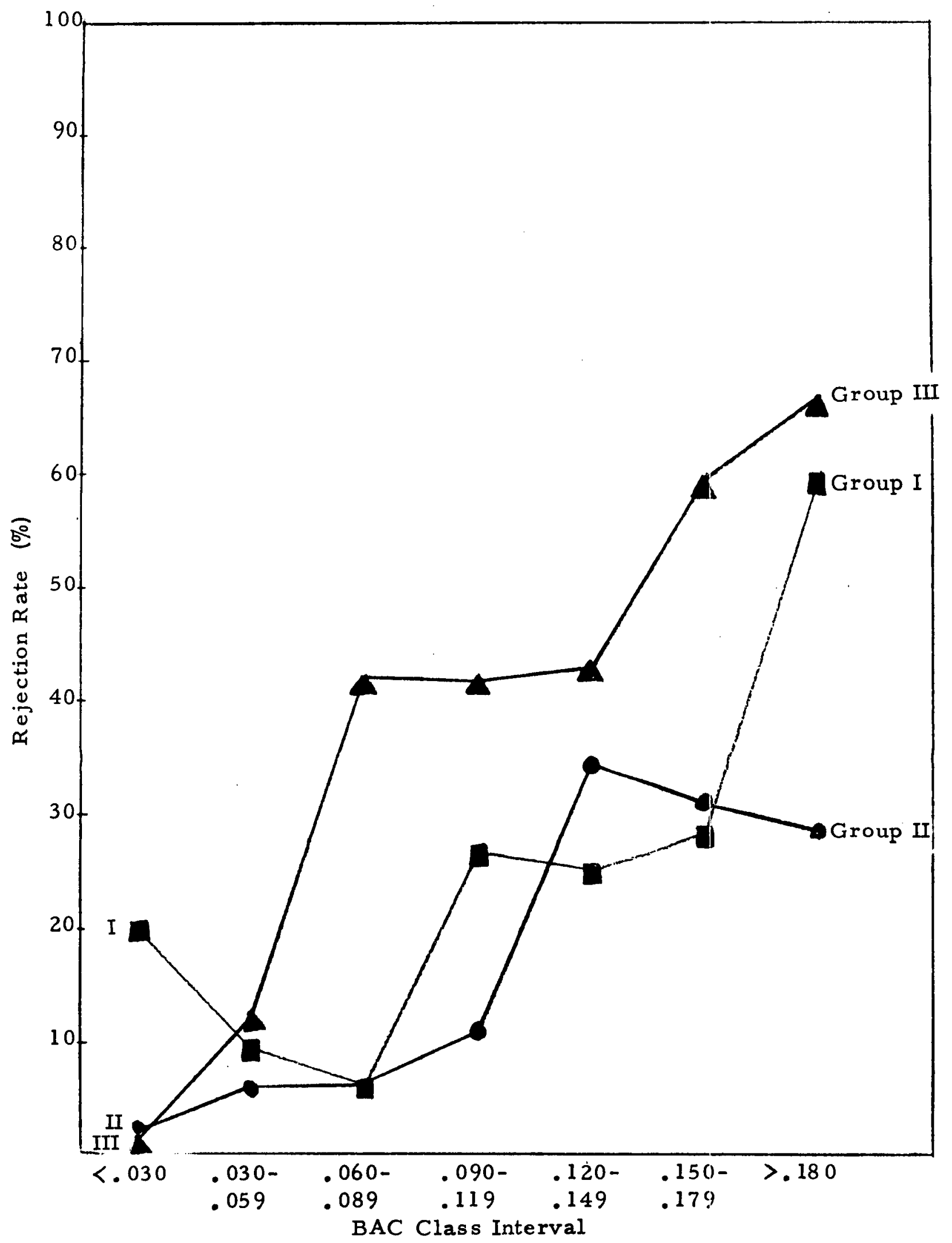

Figure 5. Phystester Performance - 2/2 Strategy 


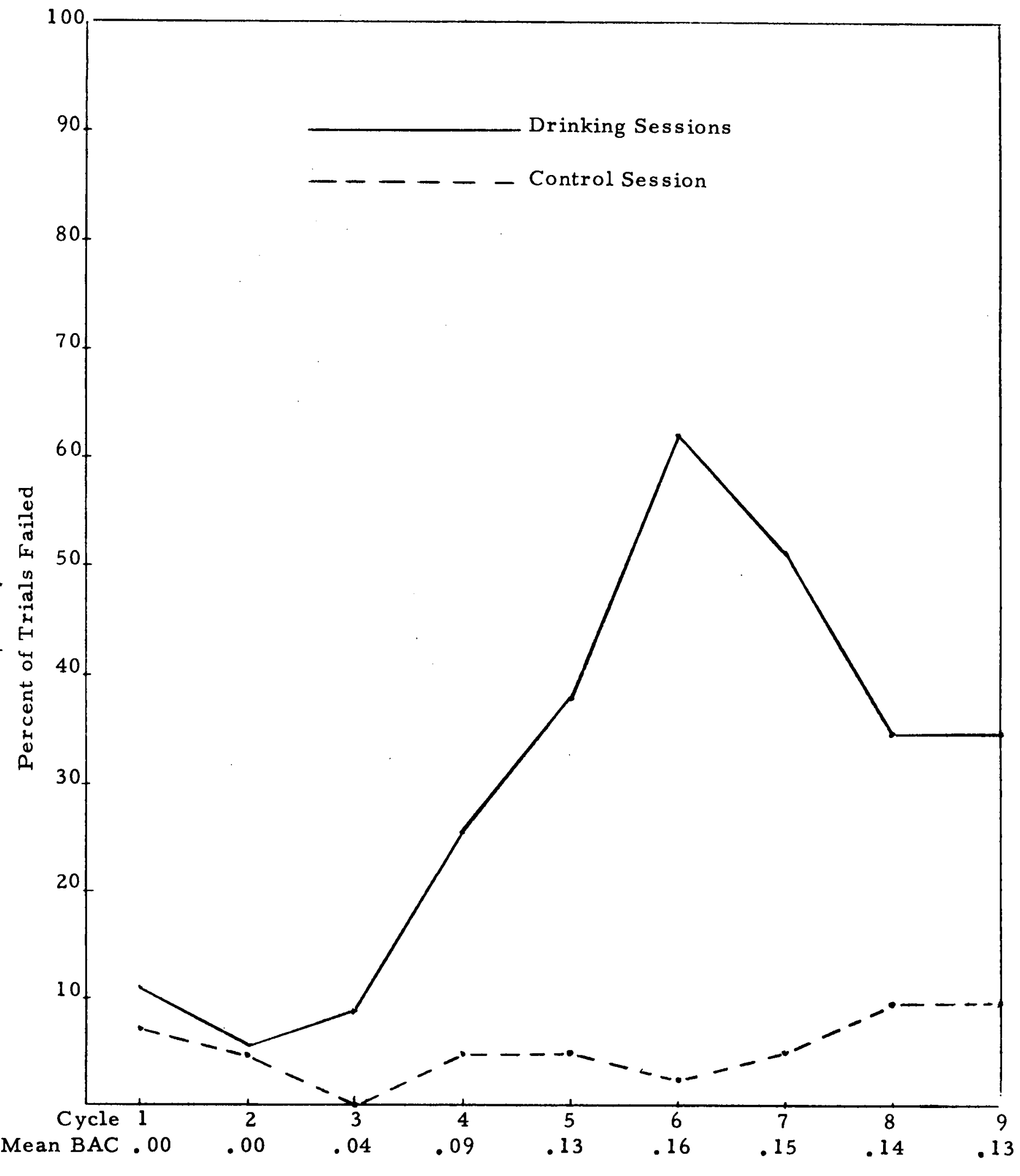

Figure 6. Phystester - Comparison of Drinking and Control Sessions $1 / 1$ Strategy - Group III 
Phystester Performance, Group I

Response time: 3.6 seconds

STRATEGY

\begin{tabular}{|c|c|c|c|c|c|c|c|c|c|}
\hline $\begin{array}{l}\text { SUBJECT } \\
\text { CATEGORY }\end{array}$ & $\begin{array}{l}\text { BAC CLASS } \\
\text { INTER VAL }\end{array}$ & \multicolumn{2}{|c|}{$1 / 1$} & \multicolumn{2}{|c|}{$3 / 3$} & \multicolumn{2}{|c|}{$2 / 3$} & \multicolumn{2}{|c|}{$2 / 2$} \\
\hline \multirow{7}{*}{$\begin{array}{l}\text { ALL } \\
\text { SUBJECTS }\end{array}$} & $<0.030 \%$ & $18 / 120$ & 15.0 & $14 / 40$ & 35,0 & $3 / 40$ & 7.5 & $8 / 40$ & 20.0 \\
\hline & $0.030-0.059 \%$ & $11 / 63$ & 17.5 & $8 / 21$ & 38.1 & $2 / 21$ & 9.5 & $2 / 21$ & 2.5 \\
\hline & $0.060-0.089 \%$ & $3 / 48$ & 6.3 & $2 / 16$ & 12.5 & $1 / 16$ & 6.3 & $1 / 16$ & 6.3 \\
\hline & $0.090-0.119 \%$ & $15 / 90$ & 16,7 & $2 / 30$ & 30,0 & $5 / 30$ & 16.7 & $8 / 30$ & 26.7 \\
\hline & $0.120-0.149 \%$ & $34 / 144$ & 23.6 & $22 / 48$ & 45.8 & $10 / 48$ & 20.8 & $12 / 48$ & 25,0 \\
\hline & $0.150-0.179 \%$ & $40 / 171$ & 23.4 & $29 / 57$ & 50.9 & $7 / 57$ & 12.3 & $16 / 57$ & 28,1 \\
\hline & $\geq 0.180 \%$ & $31 / 66$ & 47.0 & $18 / 22$ & 81.8 & $10 / 22$ & 45.5 & $13 / 22$ & 59.1 \\
\hline \multirow{7}{*}{ MALES } & $<0.030 \%$ & $9 / 54$ & 16.7 & $8 / 18$ & 44.4 & $1 / 18$ & 5.5 & $5 / 18$ & 27.8 \\
\hline & $0.030-0.059 \%$ & $8 / 30$ & 26.7 & $6 / 10$ & 60.0 & $2 / 10$ & 20.0 & $2 / 10$ & 20.0 \\
\hline & $0.060-0.089 \%$ & $1 / 27$ & 3.7 & $1 / 9$ & 11.1 & $0 / 9$ & 0.0 & $0 / 9$ & 0.0 \\
\hline & $0.090-0.119 \%$ & $6 / 33$ & 18.2 & $3 / 11$ & 27.3 & $2 / 11$ & 18.2 & $3 / 11$ & 27.3 \\
\hline & $0.120-0.149 \%$ & $20 / 69$ & 29.0 & $12 / 23$ & 52.2 & $6 / 23$ & 26.1 & $8 / 23$ & 34.8 \\
\hline & $0.150-0.179 \%$ & $19 / 93$ & 20.4 & $14 / 31$ & 45.2 & $3 / 31$ & 9.7 & $5 / 31$ & 16.1 \\
\hline & $=0.180 \%$ & $12 / 30$ & 40.0 & $7 / 10$ & 70.0 & $4 / 10$ & 40.0 & $5 / 10$ & 50.0 \\
\hline \multirow{7}{*}{ FEMALES } & $=0.030 \%$ & $9 / 66$ & 13.6 & $6 / 22$ & 27.3 & $2 / 22$ & 9.1 & $3 / 22$ & 13.6 \\
\hline & $0.030=0.059 \%$ & $3 / 33$ & 9.1 & $2 / 11$ & 18.2 & $0 / 11$ & 0.0 & $0 / 11$ & 00.0 \\
\hline & $0.060-0.089 \%$ & $2 / 21$. & 9.5 & $1 / 7$ & 14.3 & $1 / 7$ & 14.3 & $1 / 7$ & 14.3 \\
\hline & $0.090-0.119 \%$ & $9 / 57$ & 15.8 & $6 / 19$ & 31.6 & $3 / 19$ & 15.8 & $5 / 19$ & 26.3 \\
\hline & $0.120-0.149 \%$ & $14 / 75$. & 18.7 & $10 / 25$ & 40.0 & $4 / 25$ & 16.0 & $4 / 25$ & 16.0 \\
\hline & $0.150-0.179 \%$ & $21 / 78$ & 26.9 & $15 / 26$ & 57.7 & $4 / 26$ & 15.4 & $11 / 26$ & 42.3 \\
\hline & $\geq 0.180 \%$ & $19 / 36$ & 52.8 & $11 / 12$ & 8.3 & $6 / 12$ & 50.0 & $8 / 12$ & 66.7 \\
\hline \multirow{7}{*}{$\begin{array}{l}\text { AGE } \\
=30\end{array}$} & $<0.030 \%$ & $8 / 33$ & 24.2 & $5 / 11$ & 45.5 & $2 / 11$ & 18.2 & $4 / 11$ & 36.4 \\
\hline & $0.030-0.059 \%$ & $2 / 18$ & 11.1 & $2 / 6$ & 33.3 & $0 / 6$ & 0.0 & $0 / 6$ & 0.0 \\
\hline & $0.060-0.089 \%$ & $0 / 18$ & 0.0 & $0 / 6$ & 0,0 & $0 / 6$ & 0.0 & $0 / 6$ & 0.0 \\
\hline & $0.090-0.119 \%$ & $3 / 21$ & 14.3 & $2 / 7$ & 28.6 & $1 / 7$ & 14.3 & $1 / 7$ & 14.3 \\
\hline & $0.120-0.149 \%$ & $7 / 42$ & 16.6 & $4 / 14$ & 28.6 & $2 / 14$ & 14.3 & $2 / 14$ & 14.3 \\
\hline & $0.150-0.179 \%$ & $14 / 57$ & 24.6 & $9 / 19$ & 47,4 & $3 / 19$ & 15.8 & $4 / 19$ & 21.1 \\
\hline & $=0.180 \%$ & $11 / 21$ & 52.4 & $6 / 7$ & 85.7 & $4 / 7$ & 57.1 & $5 / 7$ & 71.4 \\
\hline \multirow{7}{*}{$\begin{array}{l}A G E \\
=30\end{array}$} & $=0.030 \%$ & $10 / 87$ & 11.5 & $9 / 29$ & 31.0 & $1 / 29$ & 3.4 & $4 / 29$ & 13.8 \\
\hline & $0.030-0.059 \%$ & $9 / 45$ & 20.0 & $7 / 15^{\prime}$ & 46.7 & $2 / 15$ & 13.3 & $2 / 15$ & 13.3 \\
\hline & $0.060-0.089 \%$ & $3 / 30$ & 30.0 & $2 / 10$ & 20.0 & $1 / 10$ & 10.0 & $1 / 10$ & 10.0 \\
\hline & $0.090-0.119 \%$ & $12 / 69$ & 17.4 & $7 / 23$ & 30.4 & $4 / 23$ & 17.4 & $7 / 23$ & 30.4 \\
\hline & $0.120-0.149 \%$ & $27 / 102$ & 26.5 & $18 / 34$ & 52.9 & $8 / 34$ & 23.5 & $10 / 34$ & 29.4 \\
\hline & $0.150-0.179 \%$ & $26 / 114$ & 22.8 & $20 / 38$ & 52.6 & $4 / 38$ & 10.5 & $12 / 38$ & 31.6 \\
\hline & $=0.180 \%$ & $20 / 45$ & 44.4 & $12 / 15$ & 80.0 & $6 / 15$ & 41). 0 & $8 / 15$ & 53.3 \\
\hline
\end{tabular}


Table IX

Phystester Performance - Group II Response Time: 3.6 seconds

\begin{tabular}{|c|c|c|c|c|c|c|c|c|c|c|}
\hline \multirow{3}{*}{$\begin{array}{l}\text { SUBJECT } \\
\text { CATEGORY }\end{array}$} & & \multirow{3}{*}{$\begin{array}{l}\text { CYCLE } \\
\text { TIME* }\end{array}$} & & & & & & & & \\
\hline & \multirow{2}{*}{$\begin{array}{c}\text { TEST } \\
t\end{array}$} & & \multicolumn{2}{|c|}{$1 / 1$} & \multicolumn{2}{|c|}{$3 / 3$} & \multicolumn{2}{|c|}{$2 / 3$} & \multicolumn{2}{|c|}{$2 / 2$} \\
\hline & & & $\mathrm{N}$ & $\%$ & $\mathrm{~N}$ & $\%$ & $\mathrm{~N}$ & $\%$ & $\mathrm{~N}$ & $\%$ \\
\hline \multirow{9}{*}{$\begin{array}{l}\text { ALL } \\
\text { SUBJECTS }\end{array}$} & 1. & $0: 10$ & $3 / 42$ & 7.1 & $3 / 14$ & 21.4 & $0 / 14$ & 0.0 & $0 / 14$ & 0.0 \\
\hline & 2. & $0: 35$ & $2 / 42$ & 4.8 & $2 / 14$ & 14.3 & $0 / 14$ & 0.0 & $1 / 14$ & 0.0 \\
\hline & 3. & $1: 35$ & $0 / 42$ & 0.0 & $0 / 14$ & 0.0 & $0 / 14$ & 0.0 & $0 / 14$ & 0.0 \\
\hline & 4. & $2: 35$ & $2 / 42$ & 4.8 & $2 / 14$ & 14.3 & $0 / 14$ & 0.0 & $1 / 14$ & 7.1 \\
\hline & 5. & $3: 35$ & $2 / 42$ & 4.8 & $2 / 14$ & 14.3 & $0 / 14$ & 0.0 & $0 / 14$ & 0.0 \\
\hline & 6. & $4: 35$ & $1 / 42$ & 2.4 & $1 / 14$ & 7.1 & $0 / 14$ & 0.0 & $0 / 14$ & 0.0 \\
\hline & 7. & $5: 00$ & $2 / 42$ & 4.8 & $2 / 14$ & 14.3 & $0 / 14$ & 0.0 & $0 / 14$ & 0.0 \\
\hline & 8. & $6: 00$ & $4 / 42$ & 9.5 & $3 / 14$ & 21.4 & $1 / 14$ & 7.1 & $2 / 14$ & 14.3 \\
\hline & 9. & $6: 30$ & $4 / 42$ & 9.5 & $3 / 14$ & 21.4 & $1 / 14$ & 7.1 & $1 / 14$ & 7.1 \\
\hline \multirow{9}{*}{ MALES } & 1. & $0: 10$ & $2 / 27$ & 7.4 & $2 / 9$ & 22.2 & $0 / 9$ & 0.0 & $0 / 9$ & 0.0 \\
\hline & 2. & $0: 35$ & $2 / 27$ & 7.4 & $2 / 9$ & 22.2 & $0 / 9$ & 0.0 & $1 / 9$ & 11.1 \\
\hline & 3. & $1: 35$ & $0 / 27$ & 0.0 & $0 / 9$ & 0.0 & $0 / 9$ & 0.0 & $0 / 9$ & 0.0 \\
\hline & 4. & $2: 35$ & $2 / 27$ & 7.4 & $2 / 9$ & 22.2 & $0 / 9$ & 0.0 & $1 / 9$ & 11.1 \\
\hline & 5. & $3: 35$ & $2 / 27$ & 7.4 & $2 / 9$ & 22.2 & $0 / 9$ & 0.0 & $0 / 9$ & 0.0 \\
\hline & 6. & $4: 35$ & $1 / 27$ & 3.7 & $1 / 9$ & 11.1 & $0 / 9$ & 0.0 & $0 / 9$ & 0.0 \\
\hline & 7. & $5: 00$ & $2 / 27$ & 7.4 & $2 / 9$ & 22.2 & $0 / 9$ & 0.0 & $0 / 9$ & 0.0 \\
\hline & 8. & $6: 00$ & $3 / 27$ & 11.1 & $2 / 9$ & 22.2 & $1 / 9$ & 11.1 & $2 / 9$ & 22.2 \\
\hline & 9. & $6: 30$ & $2 / 27$ & 7.4 & $2 / 9$ & 22.2 & $0 / 9$ & 0.0 & $0 / 9$ & 0.0 \\
\hline \multirow{9}{*}{ FEMALES } & 1. & $0: 10$ & $1 / 15$ & 6.7 & $1 / 5$ & 20.0 & $0 / 5$ & 0.0 & $0 / 5$ & 0.0 \\
\hline & 2. & $0: 35$ & $0 / 15$ & 0.0 & $0 / 5$ & 0.0 & $0 / 5$ & 0.0 & $0 / 5$ & 0.0 \\
\hline & 3. & $1: 35$ & $0 / 15$ & 0.0 & $0 / 5$ & 0.0 & $0 / 5$ & 0.0 & $0 / 5$ & 0.0 \\
\hline & 4. & $2: 35$ & $0 / 15$ & 0.0 & $0 / 5$ & 0.0 & $0 / 5$ & 0.0 & $0 / 5$ & 0.0 \\
\hline & 5. & $3: 35$ & $0 / 15$ & 0.0 & $0 / 5$ & 0.0 & $0 / 5$ & 0.0 & $0 / 5$ & 0.0 \\
\hline & 6. & $4: 35$ & $0 / 15$ & 0.0 & $0 / 5$ & 0.0 & $0 / 5$ & 0.0 & $0 / 5$ & 0.0 \\
\hline & 7. & $5: 00$ & $0 / 15$ & 0.0 & $0 / 5$ & 0.0 & $0 / 5$ & 0.0 & $0 / 5$ & 0.0 \\
\hline & 8. & $6: 00$ & $1 / 15$ & 6.7 & $1 / 5$ & 20.0 & $0 / 5$ & 0.0 & $0 / 5$ & 0.0 \\
\hline & 9. & $6: 30$ & $2 / 15$ & 13.3 & $1 / 5$ & 20.0 & $1 / 5$ & 20.0 & $1 / 5$ & 20.0 \\
\hline \multirow{9}{*}{$A G E<30$} & 1. & $0: 10$ & $1 / 18$ & 5.6 & $1 / 6$ & 16.7 & $0 / 6$ & 0.0 & $0 / 6$ & 0.0 \\
\hline & 2. & $0: 35$ & $1 / 18$ & 5.6 & $1 / 6$ & 16.7 & $0 / 6$ & 0.0 & $1 / 6$ & 16.7 \\
\hline & 3. & $1: 35$ & $0 / 18$ & 0.0 & 0.6 & 0.0 & $0 / 6$ & 0.0 & $0 / 6$ & 0.0 \\
\hline & 4. & $2: 35$ & $1 / 18$ & 5.6 & $1 / 6$ & 16.7 & $0 / 6$ & 0.0 & $0 / 6$ & 0.0 \\
\hline & 5. & $3: 35$ & $0 / 18$ & 0.0 & $0 / 6$ & 0.0 & $0 / 6$ & 0.0 & $0 / 6$ & 0.0 \\
\hline & 6. & $4: 35$ & $0 / 18$ & 0.0 & $0 / 6$ & 0.0 & $0 / 6$ & 0.0 & $0 / 6$ & 0.0 \\
\hline & 7. & $5: 00$ & $1 / 18$ & 5.6 & $1 / 6$ & 16.7 & $0 / 6$ & 0.0 & $0 / 6$ & 0.0 \\
\hline & 8. & $6: 00$ & $1 / 18$ & 5.6 & $1 / 6$ & 16.7 & $0 / 6$ & 0.0 & $1 / 6$ & 16.7 \\
\hline & 9. & $6: 30$ & $1 / 18$ & 5.6 & $1 / 6$ & 16.7 & $0 / 6$ & 0.0 & $0 / 6$ & 0.0 \\
\hline \multirow{9}{*}{$A G E \geq 30$} & 1. & $0: 10$ & $2 / 24$ & 8.3 & $2 / 8$ & 25.0 & $0 / 8$ & 0.0 & $0 / 8$ & 0.0 \\
\hline & 2. & $0: 35$ & $1 / 24$ & 4.2 & $1 / 8$ & 12.5 & $0 / 8$ & 0.0 & $0 / 8$ & 0.0 \\
\hline & 3. & $1: 35$ & $0 / 24$ & 0.0 & $0 / 8$ & 0.0 & $0 / 8$ & 0.0 & $0 / 8$ & 0.0 \\
\hline & 4. & $2: 35$ & $1 / 24$ & 4.2 & $1 / 8$ & 12.5 & $0 / 8$ & 0.0 & $1 / 8$ & 12.5 \\
\hline & 5. & $3: 35$ & $2 / 24$ & 8.3 & $2 / 8$ & 25.00 & $0 / 8$ & 0.0 & $0 / 8$ & 0.0 \\
\hline & 6. & $4: 35$ & $1 / 24$ & 4. 2 & $1 / 8$ & 12.5 & $0 / 8$ & 0.0 & $0 / 8$ & 0.0 \\
\hline & 7. & $5: 00$ & $1 / 24$ & 4. 2 & $1 / 8$ & 12.5 & $0 / 8$ & 0.0 & $0 / 8$ & 0.0 \\
\hline & 8. & $6: 00$ & $3 / 24$ & 12.5 & $2 / 8$ & 25.0 & $1 / 8$ & 12.5 & $1 / 8$ & 12.5 \\
\hline & 9. & $6: 30$ & $3 / 24$ & 12.5 & $2 / 8$ & 25.0 & $1 / 8$ & 12.5 & $1 / 8$ & 12.5 \\
\hline
\end{tabular}


Table X

Phystester Performance, Group II.

Response time: 3.0 seconds

STRATEGY

\begin{tabular}{|c|c|c|c|c|c|c|c|c|c|}
\hline $\begin{array}{l}\text { SUBJECT } \\
\text { CATEGORY }\end{array}$ & $\begin{array}{l}\text { BAC CLASS } \\
\text { INTER VAL }\end{array}$ & \multicolumn{2}{|c|}{$1 / 1$} & \multicolumn{2}{|c|}{$3 / 3$} & \multicolumn{2}{|c|}{$2 / 3$} & \multicolumn{2}{|c|}{$2 / 2$} \\
\hline \multirow{7}{*}{$\begin{array}{l}\text { ALL } \\
\text { SUBJECTS }\end{array}$} & $=0.030 \%$ & $14 / 177$ & 7.9 & $13 / 59$ & 22.0 & $1 / 59$ & 1.7 & $1 / 59$ & 1.7 \\
\hline & $0.030-0.059 \%$ & $6 / 72$ & 8.3 & $5 / 24$ & 20.8 & $1 / 24$ & 4.2 & $3 / 24$ & 12.5 \\
\hline & $0.060-0.089 \%$ & $16 / 57$ & 28.1 & $10 / 19$ & 52.6 & $6 / 19$ & -31.6 & $8 / 19$ & 42.1 \\
\hline & $0.090-0.119 \%$ & $34 / 108$ & 31.5 & $21 / 36$ & 58.3 & $12 / 36$ & 33.3 & $15 / 36$ & 41.7 \\
\hline & $0.120-0.149 \%$ & $51 / 147$ & 34.7 & $28 / 49$ & 57.1 & $17 / 49$ & 34.7 & $21 / 49$ & 42.9 \\
\hline & $0.150-0.179 \%$ & $75 / 156$ & 48.1 & $40 / 52$ & 76.9 & $25 / 52$ & 48.1 & $31 / 52$ & 59.6 \\
\hline & $\geq 0.180 \%$ & $36 / 63$ & 57.1 & $17 / 21$ & 81.0 & $13 / 21$ & 61.9 & $14 / 21$ & 66.7 \\
\hline \multirow{7}{*}{ MALES } & $<0.030 \%$ & $9 / 108$ & 8.3 & $8 / 36$ & 22.2 & $1 / 36$ & 2.8 & $1 / 36$ & 2.8 \\
\hline & $0.030-0.059 \%$ & $4 / 51$ & 7.8 & $3 / 17$ & 17.6 & $1 / 17$ & 5.9 & $2 / 17$ & 11.8 \\
\hline & $0.060-0.089 \%$ & $13 / 36$ & 36.1 & $8 / 12$ & 66.7 & $5 / 12$ & 41.7 & $6 / 12$ & 50.0 \\
\hline & $0.090-0.119 \%$ & $32 / 84$ & 38.1 & $19 / 28$ & 67.9 & $12 / 28$ & .42 .9 & $13 / 28$ & 46.4 \\
\hline & $0.120-0.149 \%$ & $42 / 102$ & 41.2 & $22 / 34$ & 64.7 & $15 / 34$ & 44.1 & $18 / 34$ & 52.9 \\
\hline & $0.150-0.179 \%$ & $44 / 90$ & 48.9 & $21 / 30$ & 70.0 & $16 / 30$ & 53.3 & $\begin{array}{l}18 / 30 \\
\end{array}$ & 60.0 \\
\hline & $\therefore=0.180 \%$ & $17 / 33$ & 51.5 & $9 / 11$ & 81.8 & $6 / 11$ & 54.5 & $7 / 11$ & 63.6 \\
\hline \multirow{7}{*}{ FEMALES } & $=0.030 \%$ & $5 / 69$ & 7.2 & $5 / 23$ & 21.7 & $0 / 23$ & 0.0 & $0 / 23$ & 0.0 \\
\hline & $0.030-0.059 \%$ & $2 / 21$ & 9.5 & $2 / 7$ & 28.6 & $0 / 7$ & 0.0 & $1 / 7$ & 14.3 \\
\hline & $0.060-0.089 \%$ & $3 / 21$ & 14.3 & $2 / 7$ & 28.6 & $1 / 7$ & 14.3 & $2 / 7$ & 28.6 \\
\hline & $0.090-0.119 \%$ & $2 / 24$ & 8.3 & $2 / 8$ & 25.0 & $0 / 8$ & 0.0 & $2 / 8$ & 0.0 \\
\hline & $0.120-0.149 \%$ & $9 / 45$ & 20.0 & $6 / 15$ & 40.0 & $2 / 15$ & 13.3 & $3 / 15$ & 20.0 \\
\hline & $0.150-0.179 \%$ & $31 / 66$ & 47.0 & $19 / 22$ & 86.4 & $9 / 22$ & 40.9 & $13 / 22$ & 59.1 \\
\hline & $\geq 0.180 \%$ & $19 / 30$ & 63.3 & $8 / 10$ & 80.0 & $7 / 10$ & 70.0 & $7 / 10$ & 70.0 \\
\hline \multirow{7}{*}{$\begin{array}{l}\text { AGE } \\
<30\end{array}$} & $<0.030 \%$ & $7 / 75$ & 9.3 & $7 / 25$ & 28.0 & $0 / 25$ & 0.0 & $0 / 25$ & 0.0 \\
\hline & $0.030-0.059 \%$ & $4 / 42$ & 9.5 & $4 / 14$ & 28.6 & $0 / 14$ & 0.0 & $2 / 14$ & 14.3 \\
\hline & $0.060-0.089 \%$ & $7 / 21$ & 33.3 & $5 / 7$ & 71.4 & $2 / 7$ & 28.6 & $4 / 7$ & 57.1 \\
\hline & $0.090-0.119 \%$ & $9 / 39$ & 23.1 & $7 / 13$ & 53.8 & $2 / 13$ & 15,4 & $3 / 13$ & 23.1 \\
\hline & $0.120-0.149 \%$ & $26 / 87$ & 29.9 & $16 / 29$ & 55.2 & $8 / 29$ & 27.6 & $10 / 29$ & 34.5 \\
\hline & $0.150-0.179 \%$ & $41 / 69$ & 59.4 & $20 / 23$ & 87.0 & $14 / 23$ & 60.9 & $18 / 23$ & 78.3 \\
\hline & $=0.180 \%$ & $14 / 27$ & 51.9 & $7 / 9$ & 77.8 & $5 / 9$ & 55.5 & $6 / 9$ & 66.7 \\
\hline \multirow{7}{*}{$\begin{array}{l}\text { AGE } \\
\geq 30\end{array}$} & $<0.030 \%$ & $7 / 102$ & 6.9 & $6 / 34$ & 17.6 & $1 / 34$ & 2. 9 & $1 / 34$ & 2.9 \\
\hline & $0.030-0.059 \%$ & $2 / 30$ & 6.7 & $1 / 10$ & 10.0 & $1 / 10$ & 10.0 & $1 / 10$ & 10.0 \\
\hline & $0.060-0.089 \%$ & $9 / 36$ & 25.0 & $5 / 12$ & 41.7 & $4 / 12$ & 33.3 & $4 / 12$ & 33.3 \\
\hline & $0.090-0.119 \%$ & $25 / 69$ & 36.2 & $14 / 23$ & 60.9 & $10 / 23$ & 43.5 & $12 / 23$ & 52.2 \\
\hline & $0.120-0.149 \%$ & $25 / 60$ & 41.7 & $12 / 20$ & 60.0 & $9 / 20$ & 45.0 & $11 / 20$ & 55.0 \\
\hline & $0.150-0.179 \%$ & $34 / 87$ & 39.1 & $20 / 29$ & 69.0 & $11 / 29$ & 37.9 & $13 / 29$ & 44.8 \\
\hline & $=0.180 \%$ & $22 / 36$ & 61.1 & $10 / 12$ & 83.3 & $8 / 12$ & 66.7 & $8 / 12$ & 66.7 \\
\hline
\end{tabular}


Table XI

Phystester Control Session Performance, Group III Response time : 3:0 seconds

STRA TEGY

\begin{tabular}{|c|c|c|c|c|c|c|c|c|c|}
\hline $\begin{array}{l}\text { SUBJECT } \\
\text { CATEGORY }\end{array}$ & $\begin{array}{l}\text { BAC CLASS } \\
\text { INTER VAL }\end{array}$ & \multicolumn{2}{|c|}{$1 / 1$} & \multicolumn{2}{|c|}{$3 / 3$} & \multicolumn{2}{|c|}{$2 / 3$} & \multicolumn{2}{|c|}{$2 / 2$} \\
\hline \multirow{7}{*}{$\begin{array}{l}\text { ALL } \\
\text { SUBJECTS }\end{array}$} & $=0.030 \%$ & $7 / 138$ & 5.1 & $5 / 46$ & 10.9 & $1 / 46$ & 2,2 & $1 / 46$ & 2,2 \\
\hline & $0.030-0.059 \%$ & $4 / 96$ & 4.2 & $3 / 32$ & 9.4 & $1 / 32$ & 3.1 & $2 / 32$ & 6,3 \\
\hline & $0.060-0.089 \%$ & $2 / 48$ & 4.2 & $2 / 16$ & 12.5 & $0 / 16$ & 0.0 & $1 / 16$ & 6,3 \\
\hline & $0.090-0.119 \%$ & $10 / 08$ & 9.3 & $10 / 36$ & 27.8 & $0 / 36$ & 0.0 & $4 / 36$ & 11.1 \\
\hline & $0.120-0.149 \%$ & $29 / 105$ & 27.6 & $20 / 35$ & 57.1 & $8 / 35$ & 22.9 & $12 / 35$ & 34.3 \\
\hline & $0.150-0.179 \%$ & $36 / 126$ & 28.6 & $22 / 42$ & 52.4 & $11 / 42$ & 26.2 & $13 / 42$ & 31.0 \\
\hline & $=0.180 \%$ & $24 / 84$ & 28.6 & $15 / 28$ & 53.6 & $8 / 28$ & 28.6 & $8 / 28$ & 28.6 \\
\hline \multirow{7}{*}{ MALES } & $<0.030 \%$ & $6 / 75$ & 8.0 & $4 / 25$ & 16.0 & $1 / 25$ & 4.0 & $1 / 25$ & 4.0 \\
\hline & $0.030-0.059 \%$ & $1 / 48$ & 2.1 & $1 / 16$ & 6.3 & $0 / 16$ & 0.0 & $1 / 16$ & 0.0 \\
\hline & $0.060-0.089 \%$ & $2 / 18$ & 11.1 & $2 / 6$ & 33.3 & $0 / 6$ & 0.0 & $1 / 6$ & 16.7 \\
\hline & $0.090-0.119 \%$ & $5 / 51$ & 9.8 & $5 / 17$ & 29.4 & $0 / 17$ & 0.0 & $1 / 17$ & 5.9 \\
\hline & $0.120-0.149 \%$ & $19 / 54$ & 35.2 & $12 / 18$ & 66.7 & $6 / 18$ & 33.3 & $9 / 18$ & 50.0 \\
\hline & $0.150-0.179 \%$ & $23 / 69$ & 33.3 & $13 / 23$ & 56.5 & $8 / 23$ & 34.8 & $10 / 23$ & 43.5 \\
\hline & $=0.180 \%$ & $6 / 54$ & 11.1 & $5 / 18$ & 27.8 & $1 / 18$ & 5.6 & $1 / 18$ & 5.6 \\
\hline \multirow{7}{*}{ FEMALES } & $<0.030 \%$ & $1 / 63$ & 1.6 & $1 / 21$ & 4.8 & $0 / 21$ & 0.0 & $0 / 21$ & 0.0 \\
\hline & $0.030-0.059 \%$ & $3 / 48$ & 6.3 & $2 / 16$ & 12.5 & $1 / 16$ & 6.3 & $1 / 16$ & 6.3 \\
\hline & $0.060-0.089 \%$ & $0 / 30$ & 0.0 & $0 / 10$ & 0.0 & $0 / 10$ & 0.0 & $0 / 10$ & 0.0 \\
\hline & $0.090-0.119 \%$ & $5 / 57$ & 8.8 & $5 / 19$ & 26.3 & $0 / 19$ & 0.0 & $3 / 19$ & 15.8 \\
\hline & $0.120-0.149 \%$ & $10 / 51$ & 19.6 & $8 / 17$ & 47.1 & $2 / 17$ & 11.8 & $3 / 17$ & 17,6 \\
\hline & $0.150-0.179 \%$ & $13 / 57$ & 22.8 & $9 / 19$ & 47.4 & $3 / 19$ & 15.8 & $3 / 19$ & 15,8 \\
\hline & $\geq 0.180 \%$ & $18 / 30$ & 60.0 & $10 / 10$ & 100.0 & $7 / 10$ & 70.0 & $7 / 10$ & 70.0 \\
\hline \multirow{7}{*}{$\begin{array}{l}A G E \\
=30\end{array}$} & $<0.030 \%$ & $5 / 90$ & 5.6 & $3 / 30$ & 10.0 & $1 / 30$ & 3,3 & $1 / 30$ & \\
\hline & $0.030-0.059 \%$ & $1 / 66$ & 1.5 & $1 / 22$ & 4.5 & $0 / 22$ & 0.0 & $1 / 22$ & 4.5 \\
\hline & $0.060-0.089 \%$ & $1 / 33$ & 3.0 & $1 / 11$ & 9.1 & $0 / 11$ & 0.0 & $1 / 11$ & 9.1 \\
\hline & $0.090-0.119 \%$ & $6 / 78$ & 7.7 & $6 / 26$ & 23.1 & $0 / 26$ & 0.0 & $3 / 26$ & 11.5 \\
\hline & $0.120-0.149 \%$ & $17 / 69$ & 24.6 & $12 / 23$ & 52.2 & $4 / 23$ & 17.4 & $7 / 23$ & 30.4 \\
\hline & $0.150-0.179 \%$ & $17 / 78$ & 21.8 & $11 / 26$ & 42.3 & $5 / 26$ & 19.2 & $7 / 26$ & 26.9 \\
\hline & $=0.180 \%$ & $6 / 54$ & 11.1 & $5 / 18$ & 27.8 & $1 / 18$ & 5.6 & $1 / 18$ & 5.6 \\
\hline \multirow{7}{*}{$\begin{array}{l}A G E \\
=30\end{array}$} & $=0.030 \%$ & $2 / 48$ & 4.2 & $2 / 16$ & 12.5 & $0 / 16$ & 0.0 & $0 / 16$ & 0.0 \\
\hline & $0.030-0.059 \%$ & $3 / 30$ & 10.0 & $2 / 10$ & 20.0 & $1 / 10$ & 10,0 & $1 / 10$ & 10,0 \\
\hline & $0.060-0.089 \%$ & $1 / 15$ & 6.7 & $1 / 5$ & 20.0 & $0 / 5$ & 0.0 & $0 / 5$ & \\
\hline & $0.090-0.119 \%$ & $4 / 30$ & 13.3 & $4 / 10$ & 40.0 & $0 / 10$ & 0.0 & $1 / 10$ & 10.0 \\
\hline & $0.120-0.149 \%$ & $12 / 36$ & 33.3 & $8 / 12$ & 66.7 & $4 / 12$ & 33.3 & $5 / 12$ & 41.7 \\
\hline & $0.150-0.179 \%$ & $19 / 48$ & 39.6 & $11 / 16$ & 68.8 & $6 / 16$ & 37.5 & $6 / 16$ & 37.5 \\
\hline & $=0.180 \%$ & $18 / 30$ & 60.0 & $10 / 10$ & 100.0 & $7 / 10$ & 70.0 & $7 / 10$ & 70.0 \\
\hline
\end{tabular}

* (approximate) start time of each cycle, in hours 
Table XII

Phystester Performance, Group IIIC

Response time: 3.0 seconds

\begin{tabular}{|c|c|c|c|c|c|c|c|c|c|}
\hline \multirow[b]{2}{*}{$\begin{array}{l}\text { SUBJECT } \\
\text { CATEGORY }\end{array}$} & \multirow[b]{2}{*}{$\begin{array}{l}\text { BAC C LASS } \\
\text { INTER VAL }\end{array}$} & \multicolumn{8}{|c|}{ STRATEGY } \\
\hline & & \multicolumn{2}{|r|}{$1 / 1$} & \multicolumn{2}{|r|}{$3 / 3$} & \multicolumn{2}{|r|}{$2 / 3$} & \multicolumn{2}{|c|}{$2 / 2$} \\
\hline \multirow{7}{*}{$\begin{array}{l}\text { ALL } \\
\text { SUBJECTS }\end{array}$} & $=0.030 \%$ & $5 / 33$ & 15.2 & $4 / 11$ & 36.4 & $1 / 11$ & 9.1 & $3 / 11$ & 27.3 \\
\hline & $0: 030-0.059 \%$ & $1 / 12$ & 8.3 & $1 / 4$ & 25.0 & $0 / 4$ & 0.0 & $1 / 4$ & 25.0 \\
\hline & $0.060-0.089 \%$ & $0 / 9$ & 0.0 & $0 / 3$ & 0.0 & $0 / 3$ & 0.10 & $0 / 3$ & 0.0 \\
\hline & $0.090-0.119 \%$ & $5 / 15$ & 33.3 & $3 / 5$ & 60.0 & $2 / 5$ & 40.0 & $3 / 5$ & 60.0 \\
\hline & $0.120-0.149 \%$ & $3 / 24$ & 12.5 & $2 / 8$ & 25.0 & $1 / 8$ & 12.5 & $2 / 8$ & 25.0 \\
\hline & $0.150-0.179 \%$ & $15 / 33$ & 45.5 & $8 / 11$ & 72.7 & $6 / 11$ & 54.5 & $6 / 11$ & 54.5 \\
\hline & $=0.180 \%$ & $16 / 18$ & 88.9 & $6 / 6$ & 100.0 & $6 / 6$ & 100.0 & $6 / 6$ & 100.0 \\
\hline \multirow{7}{*}{ MALES } & $<0.030 \%$ & $4 / 9$ & 44.4 & $3 / 3$ & 100.0 & $1 / 3$ & 33.3 & $2 / 3$ & 66.7 \\
\hline & $0.030-0.059 \%$ & $1 / 3$ & 33.3 & $1 / 1$ & 100.0 & $0 / 1$ & 0.0 & $1 / 1$ & 100.0 \\
\hline & $0.060-0.089 \%$ & $0 / 3$ & 0.0 & $0 / 1$ & 0.0 & $0 / 1$ & 0.0 & $0 / 1$ & 0.0 \\
\hline & $0.090-0.119 \%$ & $4 / 6$ & 66.7 & $2 / 2$ & 100.0 & $2 / 2$ & 100.0 & $2 / 2$ & 100.0 \\
\hline & $0.120-0.149 \%$ & & - & & - & & - & & 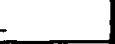 \\
\hline & $0.150-0.179 \%$ & $8 / 12$ & 66.7 & $4 / 4$ & 100.0 & $4 / 4$ & 100.0 & $4 / 4$ & 100.0 \\
\hline & $=0.180 \%$ & $14 / 15$ & 93.3 & $5 / 5$ & 100.0 & $5 / 5$ & 100.0 & $5 / 5$ & 100.0 \\
\hline \multirow{7}{*}{ FEMALES } & $=0.030 \%$ & $1 / 24$ & 4.2 & $1 / 8$ & 12.5 & $0 / 8$ & 0.0 & $1 / 8$ & 12.5 \\
\hline & $0.030-0.059 \%$ & $0 / 9$ & 0.0 & $0 / 3$ & 0.0 & $0 / 3$ & 0.0 & $0 / 3$ & 0.0 \\
\hline & $0.060-0.089 \%$ & $0 / 6$ & 0.0 & $0 / 2$ & 0.0 & $0 / 2$ & 0.0 & $0 / 2$ & 0.0 \\
\hline & $0.090-0.119 \%$ & $1 / 9$ & 11.1 & $1 / 3$ & 33.3 & $0 / 3$ & 0.0 & $1 / 3$ & 33.3 \\
\hline & $0.120-0.149 \%$ & $3 / 24$ & 12.5 & $2 / 8$ & 25.0 & $1 / 8$ & 12.5 & $2 / 8$ & 25.0 \\
\hline & $0.150-0.179 \%$ & $7 / 21$ & 33.3 & $4 / 7$ & 57.1 & $2 / 7$ & 28. 15 & $2 / 7$ & 28.6 \\
\hline & $\geq 0.180 \%$ & $2 / 3$ & 66.7 & $1 / 1$ & 100.0 & $1 / 1$ & 100.0 & $1 / 1$ & 100.0 \\
\hline \multirow{7}{*}{$\begin{array}{l}\text { AGE } \\
=30\end{array}$} & $<0.030 \%$ & $1 / 15$ & 6.7 & $1 / 5$ & 20.0 & $0 / 5$ & 0.0 & $1 / 5$ & 20,0 \\
\hline & $0.030-0.059 \%$ & $0 / 3$ & 0.0 & $0 / 1$ & 0.0 & $0 / 1$ & 0.0 & $0 / 1$ & 0.0 \\
\hline & $0.060-0.089 \%$ & $0 / 3$ & 0.0 & $0 / 1$ & 0.0 & $0 / 1$ & 0.0 & $0 / 1$ & 0.0 \\
\hline & $0.090-0.119 \%$ & $1 / 3$ & 33.3 & $1 / 1$ & 100.0 & $0 / 1$ & 0.0 & $1 / 1$ & 100.0 \\
\hline & $0.120-0.149 \%$ & $2 / 12$ & 16.7 & $1 / 4$ & 25.0 & $1 / 4$ & 25.0 & $1 / 4$ & 25.0 \\
\hline & $0.150-0.179 \%$ & $3 / 9$ & 33.3 & $2 / 3$ & 66.7 & $1 / 3$ & 33.3 & $1 / 3$ & 33.3 \\
\hline & $=0.180 \%$ & $2 / 3$ & 66.7 & $1 / 1$ & 100.0 & $1 / 1$ & 100.0 & $1 / 1$ & 100.0 \\
\hline \multirow{7}{*}{$\begin{array}{l}\text { AGE } \\
=30\end{array}$} & $=0.030 \%$ & $4 / 18$ & 22.2 & $3 / 6$ & 50.0 & $1 / 6$ & 16.7 & $2 / 6$ & 33.3 \\
\hline & $0.030-0.059 \%$ & $1 / 9$ & 11.1 & $1 / 3$ & 33.3 & $0 / 3$ & 0.0 & $1 / 3$ & 33.3 \\
\hline & $0.060-0.089 \%$ & $0 / 6$ & 0.0 & $0 / 2$ & 0.0 & $0 / 2$ & 0.0 & $0 / 2$ & 0.0 \\
\hline & $0.090-0.119 \%$ & $4 / 12$ & 33.3 & $2 / 4$ & 50.0 & $2 / 4$ & 50.0 & $2 / 4$ & 50.0 \\
\hline & $0.120-0.149 \%$ & $1 / 12$ & 8.3 & $1 / 4$ & 25.0 & $0 / 4$ & 0.0 & $1 / 4$ & 25.0 \\
\hline & $0.150-0.179 \%$ & $12 / 24$ & 50.0 & $6 / 8$ & 75.0 & $5 / 8$ & 62.5 & $5 / 8$ & 62.5 \\
\hline & $\geq 0.180 \%$ & $14 / 15$ & 93.3 & $5 / 5$ & 100.0 & $5 / 5$ & 100.0 & $5 / 5$ & 100.0 \\
\hline
\end{tabular}




\section{QUICKEY}

The Quickey, developed by Robert D. Smith, employs a micro switch/ flashing stimulus light combination. The Subject depresses the switch* to initiate a trial; as long as the switch is depressed, a red indicator is illuminated, signalling that a trial is in process. A short time later (on the order of a few seconds, with the time varying to some degree) the stimulus light flashes. As soon as the flash is noted, the Subject must immediately release the switch. A digital timer records the interval between the flash and release of the switch.

In order to pass the trial, the Subject must respond to a flash within a predetermined reaction time "window," i. e., failure will occur if the response is either too slow or too fast. The limits of the "window" are uniquely defined for each individual Subject in accordance with his baseline (training phase) reaction time record. The "window" limits are inserted into the device by means of a potentiometer calibrated in milliseconds.

"PASS" is displayed by a green indicator light, which illuminates when a response is recorded within the "window."

Details of Quickey training, testing, and analysis are presented below.

\section{A. Training Procedures}

Since the Quickey task entails merely a simple reaction to a single stimulus, it is readily learned and requires less actual training than the other ASIS units studied. However, the necessity of adjusting the pass/fail criterion to the individual Subject required the collection of substantial samples of baseline data. The circumstances under which these data were collected varied from group to group in the manner set forth below.

Group I Subjects were required to complete two Quickey blocks of $25 \mathrm{re-}$ sponses during each of their three training sessions. In an attempt to motivate Subjects to react as quickly as possible (and thus insure stringent tailoring of pass/fail criteria) a reward of $\$ 1.00$ was issued for each response less than 150 milliseconds. Of the 10 Subjects in Group $I$, three received rewards on the first training session, four on the second, and seven on the third. Three Subjects never received rewards, while two did so on all three sessions.

\footnotetext{
* Throughout this program, Subjects were required to use their left thumbs to depress the switch.
} 
Examination of Group I training results indicated that this reward system suffered from the following drawbacks:

- It seemed to create frustration among older Subjects, whose naturally slower reactions could not compete with the 150 millisecond criterion. *

- It did not necessarily motivate younger, faster Subjects adequately.

For these reasons, and in view of the fact that Quickey testing requires individually set criteria, an individually-based training reward system was implemented for Group II. Each of these Subjects completed three blocks of 25 responses on the first session. No reward could be earned on the first of these; however, the second lowest score of these 25 ( 8 th percentile) defined the $\$ 1.00$ reward criterion for the remaining two blocks. Similarly, the second lowest of that set of 50 (4th percentile) defined the criterion for the second session, during which two blocks were completed. Criterion for the third session's two blocks was established as the lesser of the 4th percentiles achieved on the first and second sessions. Under this scheme, 11 of the 12 Subjects of Group II earned rewards on the first session, and all 12 did so on both the second and third.

Quickey training for Group III differed significantly from that applied to the first two groups. In recognition of the fact that a monetary reward or other artificial motivation scheme would likely be incompatible with an operational implementation, no training criterion was defined. Group III Subjects were merely required to complete two blocks of 25 responses on each training session, without monetary incentive. However, this type of Quickey training should not be considered totally free of incentive; some of the more enthusiastic Subjects clearly were self-motivated, and probably could not have reacted appreciably faster if rewards had been offered.

The fact that Quickey pass/fail criterion must be individually set in accordance with responses scored during a baseline period suggests the possibility of cheating the instrument. It was hypothesized that a Subject could purposely misrepresent his reaction time capabilities during training and thus acquire an artificially slow pass/fail criterion which he could overcome at moderate or high BAC. During the Group III phase, four previous Subjects (two from Group I and two from Group II) were recruited to test this hypothesis. Each participated in

\footnotetext{
*The five Subjects over 40 years of age averaged $\$ 2.20$ reward over the total three session training period. The five younger Subjects alveraged $\$ 12.80$.
} 
one special training session, during which six blocks of 25 responses were recorded. These Subjects were instructed to strive not only for slow responses, but also for consistency. Thus, every attempt was made to mask the fact that cheating was taking place. The first two blocks were intended to permit the Subjects to experiment with techniques* for achieving slow, consistent responses. Once a suitable technique was found it was employed for the last four blocks, simulating the second and third training sessions. No training reward was issued to these special experiment Subjects, although each received $\$ 25$ base pay for the session.

The pass/fail criterion employed for testing each Subject of all Groups was derived solely from his responses scored on the third training session. The eighth lowest score among that set of 50 (16th percentile) defined the upper limit of the criterion "window;" when inserted into the instrument by means of the calibrated potentiometer, Quickey automatically sets $85 \%$ of the value as the lower limit. For example, a criterion of $160 \mathrm{milliseconds}$ implied that a pass would be recorded if a response were scored in the range from 136 through 160, inclusive. Criteria assigned to all Subjects are listed in Table XIII.

\section{B. Testing Procedures}

Quickey testing for Groups I, II and III was based upon a block of repeated trials of up to two minutes duration in each testing cycle. The number of responses recorded during the two minute interval typically varied from 15 to 19, largely depending upon the rapidity with which the Subject depressed the micro switch to reinstitute the test after scoring a response.

The instrument is designed so that a green indicator lights when a response falls within the criterion "window." When this occurred, the trial was halted and the Escort recorded the exact time of the pass.

Group I tested until a "green light" occurred or until the two minutes had elapsed. Thus, they had the opportunity to score no more than one pass on each cycle. Each such pass carried a $\$ 1.50$ reward. This approach unfortunately precluded conducting certain a-posteriori analyses of interest. For example, Quickey performance based upon a pass/fail scheme requiring two or more "green lights" within a two minute interval could not be estimated from these data. To overcome this limitation, Groups II and III always tested for the full two minutes. If a pass was recorded and time remained, the Escort reset the instrument and testing was resumed. Since multiple passes were possible, each carried a reward of $\$ 0.50$.

"Such as saying the word "GO" (mentally) before releasing the micro switch, and/or depressing the switch as firmly as possible while waiting for the flash. 
Table XIII

Quickey Pass/Fail Criteria

\begin{tabular}{c|c||c|c||c|c}
\hline \multicolumn{2}{c||}{ GROUP I } & \multicolumn{2}{c||}{ GROUP II } & \multicolumn{2}{c}{ GROUP III } \\
\hline Subject No. & Criterion & Subject No. & Criterion & Subject No. & Criterion \\
\hline 101 & 183 & 111 & 166 & 123 & 156 \\
102 & 140 & 112 & 164 & 125 & 177 \\
103 & 149 & 113 & 143 & 126 & 153 \\
104 & 149 & 114 & 154 & 129 & 173 \\
105 & 150 & 115 & 156 & 130 & 197 \\
106 & 167 & 116 & 172 & 131 & 178 \\
107 & 151 & 117 & 153 & 132 & 132 \\
108 & 154 & 118 & 151 & 133 & 187 \\
109 & 194 & 119 & 153 & 134 & 146 \\
110 & 175 & 120 & 135 & 135 & 148 \\
& & 121 & 141 & 136 & 196 \\
& & 122 & 174 & 138 & 181 \\
& & & & 139 & 170 \\
& & & & 140 & 165 \\
& & & & 141 & 158
\end{tabular}

Special Experiment

(Group IIIC)

\begin{tabular}{|c|c|}
\hline Subject No.* & Criterion \\
\hline $124 \quad(114)$ & 189 \\
\hline $127 \quad(107)$ & 195 \\
\hline $128(108)$ & 187 \\
\hline 137 (117) & 197 \\
\hline
\end{tabular}

*Numbers in parentheses are the original ID numbers assigned to the special experiment Subjects. 
Special experiment Subjects participated in two test sessions conducted separately from the Group III members. Their test cycles consisted of two Quickey blocks, separated by Phystester and Complex Reaction Tester blocks. They received rewards on all three instruments in accordance with the Group III procedures. Illness forced one of these four Subjects (No. 124) to withdraw from the program prior to testing.

A critical problem was experienced during testing of all groups. The green light occasionally would illuminate when the response was outside the criterion "window" (at times by a wide margin), and, conversely, occasionally would fail to illuminate for a satisfactory response. This problem destroyed much of the value of the pass times recorded by the Escorts. * Since static charges seemed to contribute to this problem, a sheet of hardboard was placed under the testing table and Subject's chair. However, this did not eliminate the problem.

Notwithstanding its inaccuracies, illumination of the green light governed all reward payments.

\section{Analyses and Results}

Strategies applicable to Quickey differ from the general form requiring $N$ or more passes out of a fixed set of $M$ trials. Instead, the parameters of interest are:

- The width of the criterion response "window;"

- The location of the "window" with respect to the distribution of training reaction time scores;

- The amount of trial time permitted;

- The number of within-"window" scores that must be recorded to achieve a pass.

Four "windows" were selected initially for analysis. Their upper limits were defined, respectively, by the $12 \mathrm{th}, 16 \mathrm{th}, 20 \mathrm{th}$, and $24 \mathrm{th}$ percentile scores produced by each Subject during his third training session; ** lower limits were set at $85 \%$ of the upper limit value in each case. Trial time was set at two minutes. These parameters were then applied to the following strategies:

It was impossible to determine subsequently whether a recorded time corresponds to a valid or invalid "pass."

i. e., the sixth lowest of the third training session's 50 scores defined the $12 t$, percentile, the eighth lowest the 16th, etc. 
- At least one criterion response* per trial at 12 th percentile (1-12th)

- At least one criterion response per trial at 16th percentile (1-16th)

- At least two criterion responses per trial at 16 th percentile $(2-16$ th $)$

- At least one criterion response per trial at 20th percentile (1-20th)

- At least one criterion response per trial at 24 th percentile $(1-24 \mathrm{th})$

- At least two criterion responses per trial at 24 th percentile $(2-24$ th $)$

Group I testing called for cessation of a trial as soon as a response fell within the 16th percentile "window." The resulting scarcity of data limited the number of strategies under which that group's performance could be examined. Table XIV, which documents Group I results, thus includes analyses only of the 1-16th, 1-20th, and 1-24th strategies. As mentioned previously, the green light occasionally illuminated (causing termination of a Group I trial) without a previous criterion response. Such trials were treated as neither passes nor fails under the 1-16th strategy but were simply disregarded for the analysis. In some of these cases, 20th and/or 24th percentile responses had been recorded before premature termination of the trial, permitting inclusion of such trials in the analysis of those strategies.

Groups II and III always tested for the full two minutes of every trial, so their data could be assessed under all strategies. Results are shown in Tables XV and XVI; Group III control session results are presented in Table XVII. Performance corresponding to the 1-16th, 1-20th, and 1-24th strategies for all three groups is depicted in Figure 7, 8, and 9, respectively.

Substantially different performance was exhibited by the three groups, with Group II producing the highest rejection rates at all BAC intervals and Group III generally the lowest. Analysis of variance under the 1-16th strategy indicates that the difference between these two groups is significant $(p<.01)$. The most plausible explanation for this difference perhaps lies in the various training paradigms employed. Group II Subjects, it will be recalled, essentially were required to continually decrease their reaction times in order to achieve training rewards, and thus tended to be driven to the limits of their capabilities; Group I Subjects only needed to surpass the $150 \mathrm{msec}$ target, which was certainly well within the capability of some individuals; members of Group III were driven only in relation to the strength of their own self-motivation. The financially-based training motivation thus may have produced overly stringent testing criteria. This hypothesis is strengthened by the very high rejection rates produced by Groups I and II at low BAC. However, even the relatively less exacting criteria assigned to Group III produced undesirably high failure rates at zero BAC.

i. e., within-"window" score. 


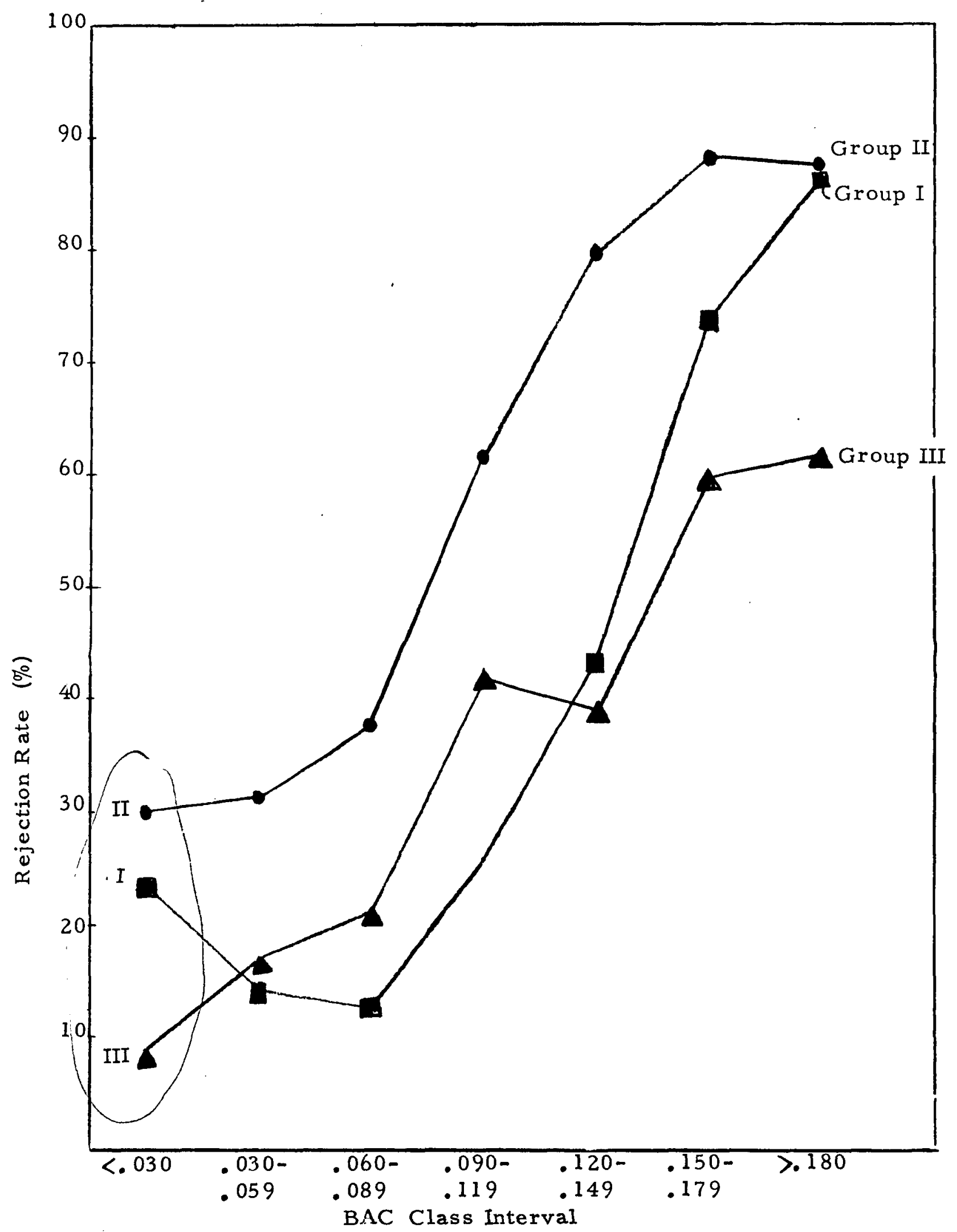

Figure 7. Quickey Performance - 1-16th Strategy 


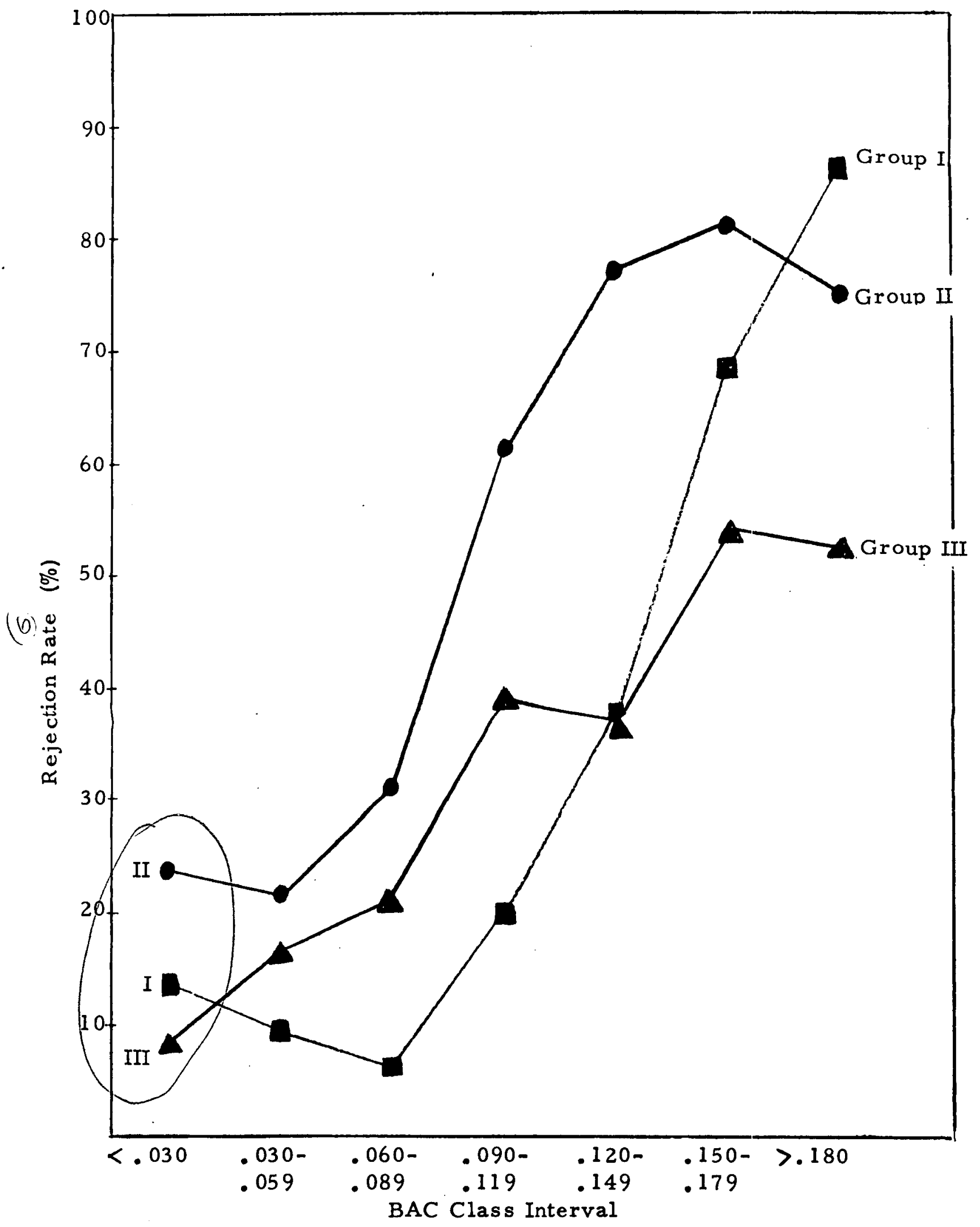

Figure 8. Quickey Performance - 1-20th Strategy 


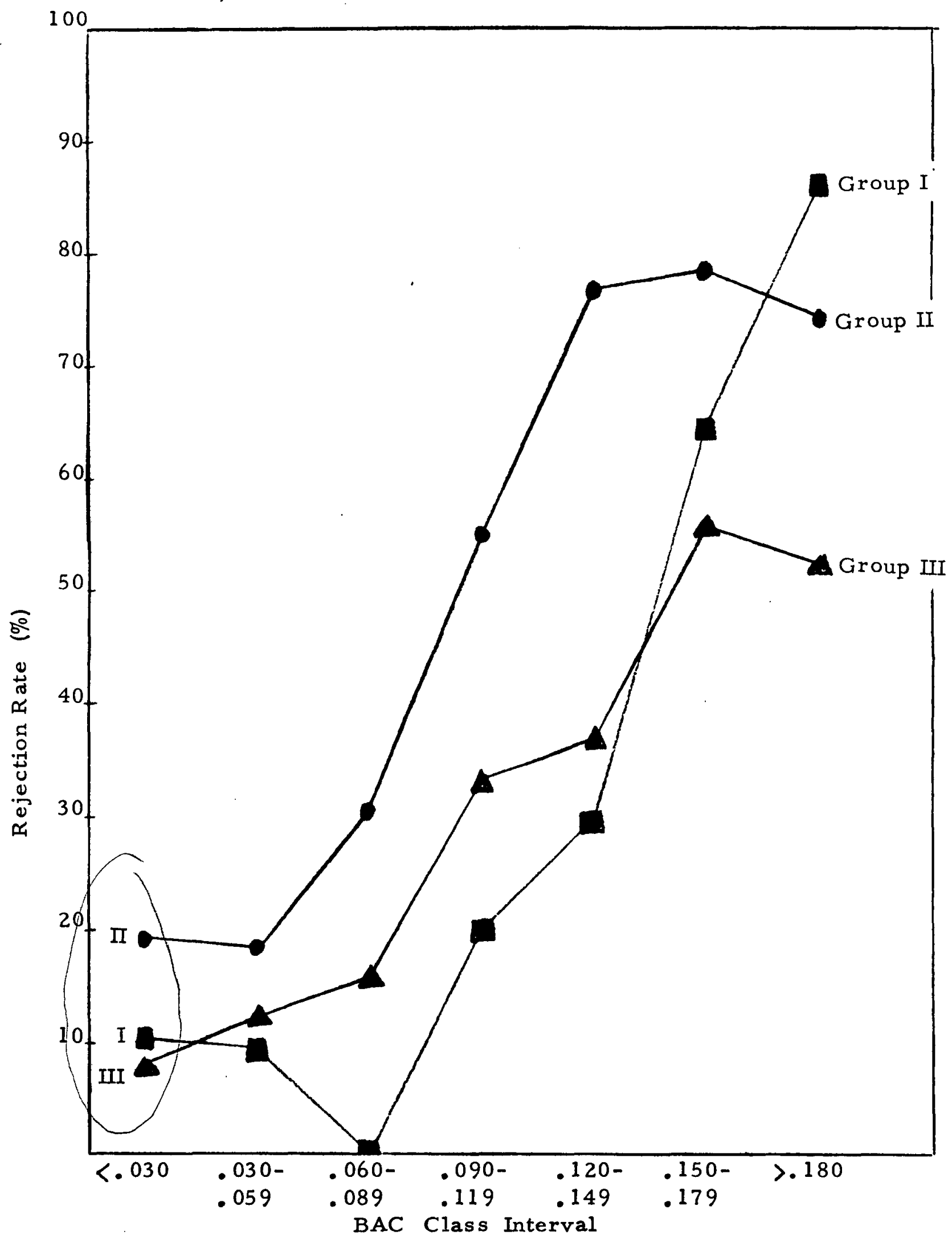

Figure 9. Quickey Performance - 1-24th Strategy 
Performance under the 1-16th strategy was subjected to analyses of variance of $B A C$ versus sex and $B A C$ versus age. The results (listed below) indicate no significant differences with respect to sex $(p \rightarrow 10)$. However, Groups I and II exhibit significant differences with respect to age $(p<.01)$; in the se two groups, younger Subjects (age $<30$ ) had generally lower testing criteria than their older counterparts and correspondingly higher rejection rates. The lack of significant difference with respect to age demonstrated by Group III may well be due to, the fact that the eldest memiber of that group was 39 years of age.

\begin{tabular}{|c|c|c|c|c|c|}
\hline & \multicolumn{2}{|c|}{ BAC vs. SEX } & \multicolumn{2}{|c|}{ BAC vs. AGE } \\
\hline & & $\mathrm{BAC}$ & SEX & BAC & $A G E$ \\
\hline$\underset{\text { I }}{\text { Group }}$ & $\begin{array}{l}F \\
\text { Significance }\end{array}$ & $\begin{array}{c}7.02 \\
.05>p=.01\end{array}$ & $\begin{array}{c}1.88 \\
p=.20\end{array}$ & $\begin{array}{c}13.13 \\
.01>p>.001\end{array}$ & $\begin{array}{l}16.47 \\
.01=p=.001\end{array}$ \\
\hline $\begin{array}{l}\text { Group } \\
\text { II }\end{array}$ & $\begin{array}{l}F \\
\text { Significance }\end{array}$ & $\begin{array}{c}8.27 \\
.05>p>.01\end{array}$ & $\begin{array}{c}3.30 \\
.20=p>.10\end{array}$ & $\begin{array}{l}32.58 \\
.001>p\end{array}$ & $\begin{array}{c}34.72 \\
.01>p>.001\end{array}$ \\
\hline $\begin{array}{l}\text { Group } \\
\text { III }\end{array}$ & $\begin{array}{l}F \\
\text { Significance }\end{array}$ & $\begin{array}{c}5.64 \\
.05=p=.01\end{array}$ & $\begin{array}{l}.07 \\
\text { N. S. }\end{array}$ & $\begin{array}{l}24.98 \\
.001>p\end{array}$ & $\begin{array}{c}14 \\
\text { N. S. }\end{array}$ \\
\hline
\end{tabular}

The major conclusion to be drawn from these analyses is that the various strategies thus far examined are inadequate for operational applications of Quickey, owing to the high rejection rates at low BAC. To further explore the performance of this device, additional analyses were conducted using a revised criterion assignment technique. This consisted merely of boosting the limits of the previously established 16th percentile "window" by 10\%. Group II and Group III data were re-examined relative to this new "window" under strategies calling for:

- At least one criterion response, and

- At least two such responses.

Results are shown in Table XVIII, and in Figures 10 and 11 . It is evident that the relatively slight adjustment in criterion has a major beneficial effect upon Quickey performance, with the data indicating that the instrument might be quite attractive for operational use under a strategy requiring at least two responses within this adjusted criterion "window." However, the extreme sensitivity of performance implies that great care must be exercised in assigning criteria to the drivers involved in such applications. This need is manifested 


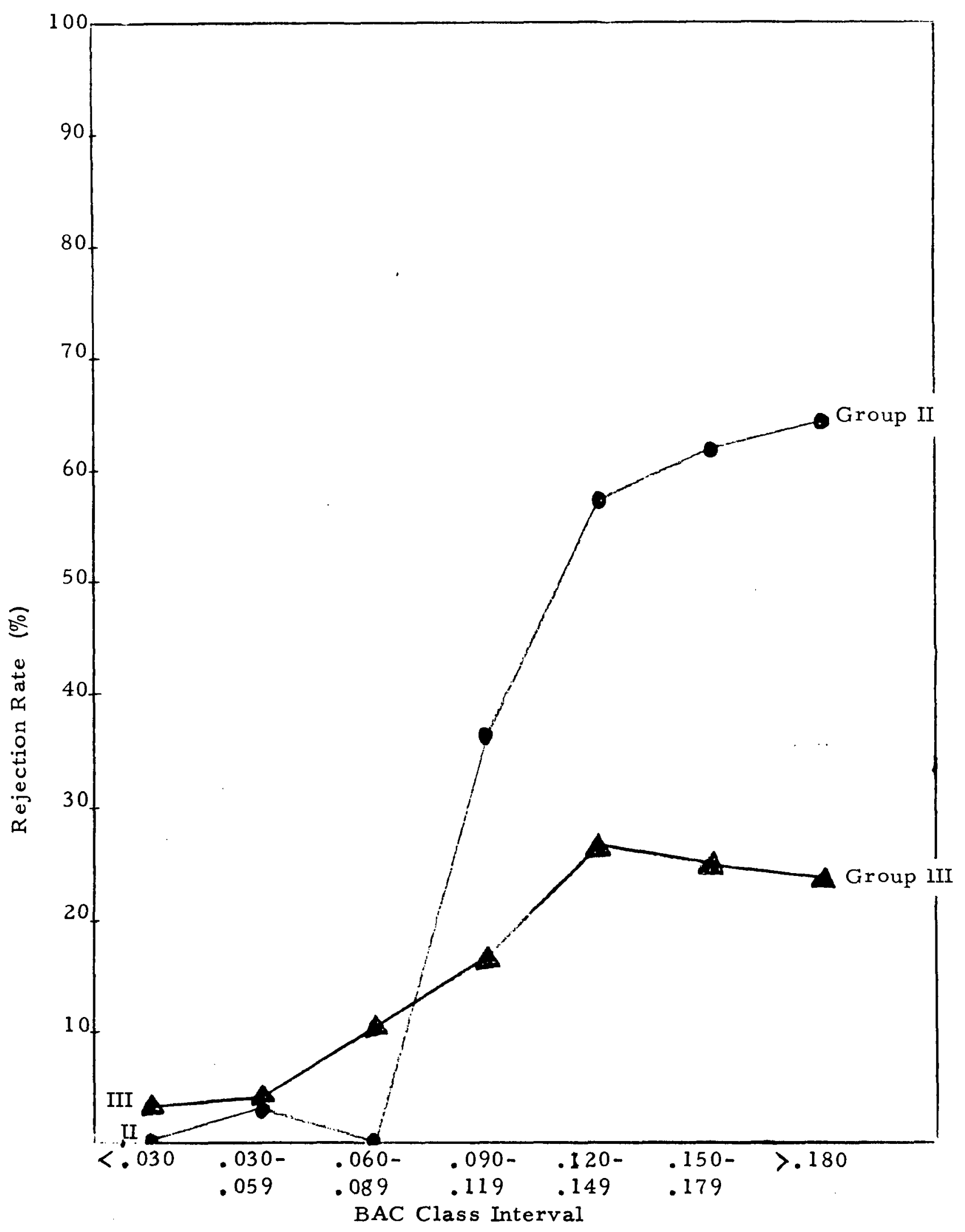

Figure 10. Quickey - Adjusted Criterion Performance, One Response 


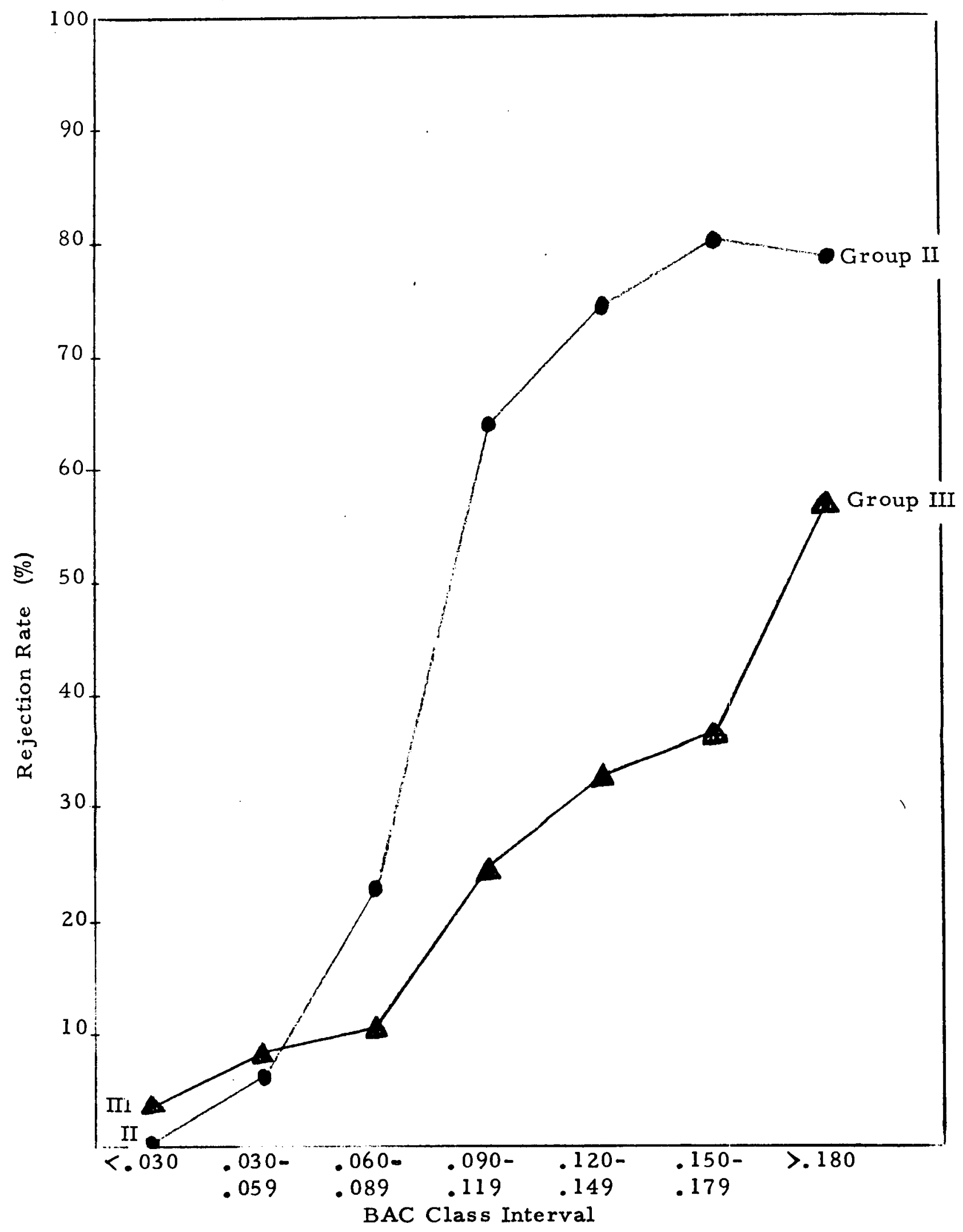

Figure 11. Quickey - Adjusted Criterion Performance, Two Responses 
even more clearly in the results obtained from the special experiment Subjects (see Table XIX). Those individuals exhibited negligible rejection rates at all $B A C$ intervals, notwithstanding the fact that their testing criteria were not appreciably different from those of many of the Group III Subjects. As a result, the possibility that Quickey can be "cheated" successfully cannot be discounted; at the very least, this possibility will further compound the problem of assigning adequate criteria.

Control session data indicate that Quickey performance, like that of the Complex Reaction Tester and the Phystester, is susceptible to the effects of the anxiety presumably present at the beginning of the session. It is of interest to note that rejection rates produced during the first two cycles of control sessions are a great deal higher than those noted during the same portion of drinking sessions. * No explanation has yet been hypothesized for this phenomenon.

Figure 12 compares control and drinking session performance at each test cycle under the 1-16th strategy. The mean BAC of each drinking session cycle is also denoted. Analysis of variance on these data indicates that neither main effect for Treatment (Controls vs. Drinking) nor Cycle is significant at the $95 \%$ level of confidence. The large interaction apparent in the curves of Figure 12 masks both main effects and since it served as the "error" term in this analysis it is not testable for significance.

*i. e., prior to the ingestion of alcohol. 


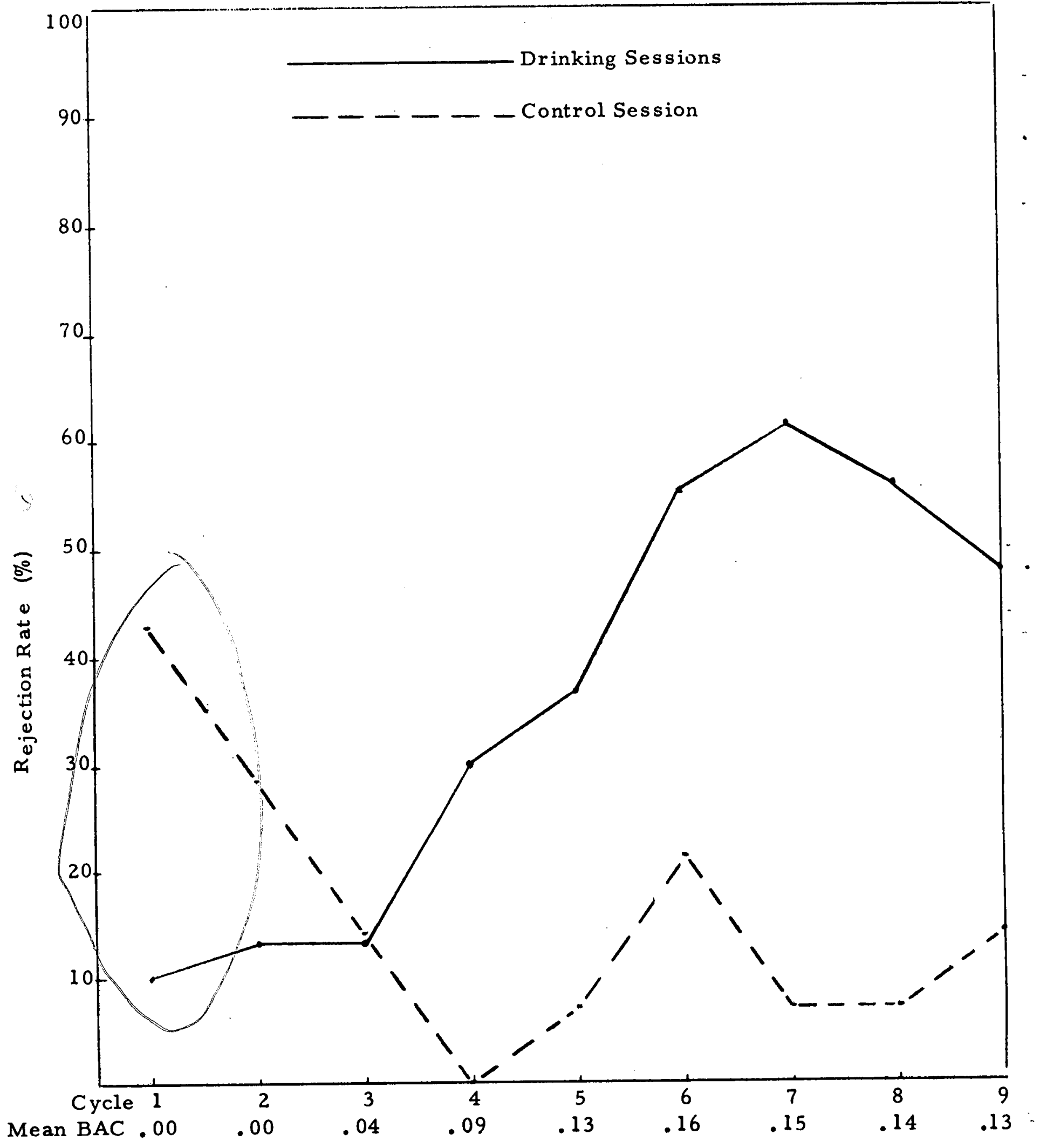

Figure 12. Quickey - Comparison of Drinking and Control Sessions 1-16th Strategy - Group III 
Table XIV

Quickey Performance, Group I

STRATEGY

\begin{tabular}{|c|c|c|c|c|c|c|c|c|c|}
\hline $\begin{array}{l}\text { SUBJECT } \\
\text { CATEGORY } \\
\end{array}$ & $\begin{array}{l}\text { BAC CLASS } \\
\text { INTERVAL }\end{array}$ & $\mathrm{N}$ & $-16 \mathrm{th} \%$ & $\mathrm{~N}^{1-2}$ & th & $\mathrm{N}^{1-24}$ & th & $\mathrm{N}$ & $\%$ \\
\hline \multirow{7}{*}{$\begin{array}{l}\text { ALL } \\
\text { SUBJECTS }\end{array}$} & $<0.030 \%$ & $8 / 34$ & 23.5 & $5 / 36$ & 13.9 & $4 / 39$ & 10.3 & & \\
\hline & $0: 030-0.059 \%$ & $3 / 21$ & 14.3 & $2 / 21$ & 9.5 & $2 / 21$ & 9.5 & & \\
\hline & $0.060-0.089 \%$ & $2 / 16$ & 12.5 & $1 / 16$ & 6.3 & $0 / 16$ & 0.0 & & \\
\hline & $0.090-0.119 \%$ & $7 / 27$ & 25.9 & $6 / 30$ & 20.0 & $6 / 30$ & 20.0 & & \\
\hline & $0.120-0.149 \%$ & $20 / 46$ & 43.5 & $18 / 48$ & 37.5 & $14 / 47$ & 29.8 & & \\
\hline & $0.150-0.179 \%$ & $42 / 57$ & 73.7 & $39 / 57$ & 68.4 & $37 / 57$ & 64.9 & & \\
\hline & $\geq 0.180 \%$ & $19 / 22$ & 86.4 & $19 / 22$ & 86.4 & $19 / 22$ & 86.4 & & \\
\hline \multirow{7}{*}{ MALES } & $<0.030 \%$ & $2 / 12$ & 16.7 & $1 / 14$ & 7.1 & $1 / 16$ & 6.3 & & \\
\hline & $0.030-0.059 \%$ & $1 / 10$ & 10.0 & $1 / 10$ & 10.0 & $1 / 10$ & 10.0 & & \\
\hline & $0.060-0.089 \%$ & $1 / 9$ & 11.1 & $0 / 9$ & 0.0 & $0 / 9$ & 0.0 & & \\
\hline & $0.090-0.119 \%$ & $4 / 9$ & 44.4 & $3 / 11$ & 27.3 & $3 / 11$ & 27.3 & & \\
\hline & $0.120-0.149 \%$ & $9 / 21$ & 42.9 & $9 / 23$ & 39.1 & $7 / 23$ & 30.4 & & \\
\hline & $0.150-0.179 \%$ & $26 / 31$ & 83.9 & $24 / 31$ & 77.4 & $23 / 31$ & 74.2 & & \\
\hline & $=0.180 \%$ & $10 / 10$ & 100.0 & $10 / 10$ & 100.0 & $10 / 10$ & 100.0 & & \\
\hline \multirow{7}{*}{ FEMALES } & $<0.030 \%$ & $6 / 22$ & 27.3 & $4 / 22$ & 18.2 & $3 / 22$ & 13.6 & & \\
\hline & $0.030-0.059 \%$ & $2 / 11$ & 18.2 & $1 / 11$ & 9.1 & $1 / 11$ & 9.1 & & \\
\hline & $0.060-0.089 \%$ & $1 / 7$ & 14.3 & $1 / 7$ & 14.3 & $0 / 7$ & 0.0 & & \\
\hline & $0.090-0.119 \%$ & $3 / 18$ & 16.7 & $3 / 19$ & 15.8 & $3 / 19$ & 15.8 & & \\
\hline & $0.120-0.149 \%$ & $11 / 25$ & 44.0 & $9 / 25$ & 36.0 & $7 / 24$ & 28.0 & & \\
\hline & $0.150-0.179 \%$ & $16 / 26$ & 61.5 & $15 / 26$ & 57.7 & $14 / 26$ & 53.8 & & \\
\hline & $\geq 0.180 \%$ & $9 / 12$ & 75.0 & $9 / 12$ & 75.0 & $9 / 12$ & 75.0 & & \\
\hline \multirow{7}{*}{$\begin{array}{l}\text { AGE } \\
<30\end{array}$} & $<0.030 \%$ & $3 / 8$ & 37.5 & $2 / 10$ & 20.0 & $2 / 11$ & 18.2 & & \\
\hline & $0.030-0.059 \%$ & $1 / 6$ & 16.7 & $1 / 6$ & 16.7 & $1 / 6$ & 16.7 & & \\
\hline & $0.060-0.089 \%$ & $2 / 6$ & 33.3 & $1 / 6$ & 16.7 & $0 / 6$ & 0.0 & & \\
\hline & $0.090-0.119 \%$ & $2 / 5$ & 40.0 & $2 / 7$ & 28.6 & $2 / 7$ & 28.6 & & \\
\hline & $0.120-0.149 \%$ & $10 / 13$ & 76.9 & $10 / 14$ & 71.4 & $8 / 14$ & 57.1 & & \\
\hline & $0.150-0.179 \%$ & $18 / 19$ & 94.7 & $17 / 19$ & 89.5 & $17 / 19$ & 89.5 & & \\
\hline & $\geq 0.180 \%$ & $7 / 7$ & 100.0 & $7 / 7$ & 100.0 & $7 / 7$ & 100.0 & & \\
\hline \multirow{7}{*}{$\begin{array}{l}A G E \\
=30\end{array}$} & $<0.030 \%$ & $5 / 26$ & 19.2 & $3 / 26$ & 11.5 & $2 / 28$ & 7.1 & & \\
\hline & $0.030-0.059 \%$ & $2 / 15$ & 13.3 & $1 / 15$ & 6.7 & $1 / 15$ & 6.7 & & \\
\hline & $0.060-0.089 \%$ & $0 / 10$ & 0.0 & $0 / 10$ & 0.0 & $0 / 10$ & 0.0 & & \\
\hline & $0.090-0.119 \%$ & $5 / 22$ & 22.7 & $4 / 23$ & 17.4 & $4 / 23$ & 17.4 & & \\
\hline & $0.120-0.149 \%$ & $10 / 33$ & 30.3 & $8 / 34$ & 23.5 & $6 / 33$ & 18.2 & & \\
\hline & $0.150-0.179 \%$ & $24 / 38$ & 63.2 & $22 / 38$ & 57.9 & $20 / 38$ & 52.6 & & \\
\hline & $=0.180 \%$ & $12 / 15$ & 80.0 & $12 / 15$ & 80.0 & $12 / 15$ & 80.0 & & \\
\hline
\end{tabular}


STRATEGY

\begin{tabular}{|c|c|c|c|c|c|c|c|c|c|c|c|c|c|}
\hline \multirow{2}{*}{$\begin{array}{l}\text { SUBJECT } \\
\text { CATEGORY }\end{array}$} & \multirow{2}{*}{$\begin{array}{l}\text { BAC CLASS } \\
\text { INTER VAL }\end{array}$} & \multirow{2}{*}{\multicolumn{2}{|c|}{$1 / 12$ th $\%$}} & \multicolumn{2}{|c|}{$1-16 \mathrm{th}$} & \multicolumn{2}{|c|}{$2-16$ th } & \multicolumn{2}{|c|}{$1-20$ th } & \multicolumn{2}{|c|}{$2 / 24$} & \multicolumn{2}{|c|}{$1 / 24$} \\
\hline & & & $\%$ & $\mathbf{N}$ & $\%$ & $\mathrm{~N}$ & $\%$ & $\mathbf{N}$ & & $\mathbf{N}$ & & & \\
\hline \multirow{7}{*}{$\begin{array}{l}\text { ALL } \\
\text { SUBJECTS }\end{array}$} & $=0.030 \%$ & $20 / 46$ & 43.5 & $14 / 46$ & 30,4 & $25 / 46$ & 54.3 & $11 / 46$ & 23.9 & $15 / 46$ & 32.6 & $9 / 46$ & 19.6 \\
\hline & $0.030-0.059 \%$ & $17 / 32$ & 53.1 & $10 / 32$ & 31.3 & $19 / 32$ & 59.4 & $7 / 32$ & 21.9 & $17 / 32$ & 53,1 & $6 / 32$ & 18.8 \\
\hline & $0.060-0.089 \%$ & $6 / 16$ & 37.5 & $6 / 16$ & 37.5 & $10 / 16$ & 62.5 & $5 / 16$ & 31.3 & $6 / 16$ & 37.5 & $5 / 16$ & 31.3 \\
\hline & $0.090-0.119 \%$ & $24 / 36$ & 66.7 & $22 / 36$ & 61.1 & $31 / 36$ & 86.1 & $22 / 36$ & 61.1 & $30 / 36$ & 83.3 & $20 / 36$ & 55.6 \\
\hline & $0.120-0.149 \%$ & $29 / 35$ & 82.9 & $28 / 35$ & 80.0 & $31 / 35$ & 88.6 & $27 / 35$ & 77.1 & $30 / 35$ & 85.7 & $27 / 35$ & 77.1 \\
\hline & $0.150-0.179 \%$ & $37 / 42$ & 88.1 & $37 / 42$ & 88.1 & $42 / 42$ & 100.0 & $34 / 42$ & 81.0 & $40 / 42$ & 95.2 & $33 / 42$ & 78.6 \\
\hline & $\geq 0.180 \%$ & $24 / 28$ & 85.7 & $23 / 28$ & 82.1 & $26 / 28$ & 92.9 & $21 / 28$ & 75.0 & $25 / 28$ & 89.3 & $21 / 28$ & 75.0 \\
\hline \multirow{7}{*}{ MALES } & $<0.030 \%$ & $10 / 25$ & 40.0 & $6 / 25$ & 24.0 & $15 / 25$ & 60.0 & $4 / 25$ & 16.0 & $8 / 25$ & 32.0 & $3 / 25$ & 12.0 \\
\hline & $0.030-0.059 \%$ & $9 / 16$ & 56.3 & $5 / 16$ & 31.3 & $11 / 16$ & 68.8 & $4 / 16$ & 25.0 & $10 / 16$ & 62.5 & $3 / 16$ & 18.8 \\
\hline & $0.060-0.089 \%$ & $3 / 6$ & 50.0 & $3 / 6$ & 50.0 & $4 / 6$ & 66.7 & $3 / 6$ & 50.0 & $3 / 6$ & 50.0 & $3 / 6$ & 50.0 \\
\hline & $0.090-0.119 \%$ & $12 / 17$ & 70.6 & $11 / 17$ & 64.7 & $5 / 17$ & 29.4 & $11 / 17$ & 64.7 & $15 / 17$ & 88.2 & $10 / 17$ & 58.8 \\
\hline & $0.120-0.149 \%$ & $17 / 18$ & 94.4 & $17 / 18$ & 94.4 & $17 / 18$ & 94.4 & $17 / 18$ & 94.4 & $17 / 18$ & 94.4 & $17 / 18$ & 94.4 \\
\hline & $0.150-0.179 \%$ & $22 / 23$ & 95.7 & $22 / 23$ & 95.7 & $23 / 23$ & 100.0 & $20 / 23$ & 87,0 & $22 / 23$ & 95.7 & $19 / 23$ & 82.6 \\
\hline & $=0.180 \%$ & $16 / 18$ & 88.9 & $15 / 18$ & 83.3 & $17 / 18$ & 94.4 & $13 / 18$ & 72.2 & $16 / 18$ & 88.9 & $13 / 18$ & 72.2 \\
\hline \multirow{7}{*}{ FEMALES } & $<0.030 \%$ & $10 / 21$ & 47.6 & $8 / 21$ & 38.1 & $10 / 21$ & 47.6 & $7 / 21$ & 33.3 & $7 / 21$ & 33.3 & $6 / 21$ & 28.6 \\
\hline & $0.030-0.059 \%$ & $8 / 16$ & 50.0 & $5 / 16$ & 31.3 & $8 / 16$ & 50.0 & $3 / 16$ & 18.8 & $7 / 16$ & 43.8 & $3 / 16$ & 18.8 \\
\hline & $0.060-0.089 \%$ & $3 / 10$ & 30.0 & $3 / 10$ & 30.0 & $6 / 10$ & 60.0 & $2 / 10$ & 20.0 & $3 / 10$ & 30.0 & $2 / 10$ & 80.0 \\
\hline & $0.090-0.119 \%$ & $12 / 19$ & 63.2 & $11 / 19$ & 57.9 & $16 / 19$ & 84.2 & $11 / 19$ & 57.9 & $15 / 19$ & 78.9 & $10 / 19$ & 52.6 \\
\hline & $0.120-0.149 \%$ & $12 / 17$ & 70.6 & $11 / 17$ & 64.7 & $14 / 17$ & 82.4 & $10 / 17$ & 58.8 & $13 / 17$ & 76.5 & $10 / 17$ & 58.8 \\
\hline & $0.150-0.179 \%$ & $15 / 19$ & 78.9 & $15 / 19$ & 78.9 & $19 / 19$ & 100.0 & $14 / 19$ & 73.7 & $18 / 19$ & 94.7 & $14 / 19$ & 73.7 \\
\hline & $=0.180 \%$ & $8 / 10$ & 80.0 & $8 / 10$ & 80.0 & $9 / 10$ & 90.0 & $8 / 10$ & 80.0 & $9 / 10$ & 90.0 & $8 / 10$ & 80.0 \\
\hline \multirow{7}{*}{$\begin{array}{l}\text { AGE } \\
<30\end{array}$} & $=0.030 \%$ & $14 / 30$ & 46.7 & $12 / 30$ & 40.0 & $20 / 30$ & 66,7 & $9 / 30$ & 30.0 & $13 / 30$ & 43,3 & $8 / 30$ & 26.7 \\
\hline & $0.030-0.059 \%$ & $14 / 22$ & 63.6 & $9 / 22$ & 40.9 & $16 / 22$ & 72.7 & $6 / 22$ & 27.3 & $14 / 22$ & 63.6 & $5 / 22$ & 22.7 \\
\hline & $0.060-0.089 \%$ & $5 / 11$ & 45.5 & $5 / 11$ & 45.5 & $8 / 11$ & 72,7 & $5 / 11$ & 45,5 & $6 / 11$ & 54.5 & $5 / 11$ & 45.5 \\
\hline & $0.090-0.119 \%$ & $19 / 26$ & 73.1 & $17 / 26$ & 65.4 & $24 / 26$ & 92,3 & $17 / 26$ & 65.4 & $23 / 26$ & 88,5 & $15 / 26$ & 57.7 \\
\hline & $0.120-0.149 \%$ & $21 / 23$ & 91.3 & $20 / 23$ & 87.0 & $22 / 23$ & 95.7 & $20 / 23$ & 87.0 & $22 / 23$ & 95,7 & $20 / 23$ & 87.0 \\
\hline & $0.150-0.179 \%$ & $25 / 26$ & 96.2 & $25 / 26$ & 96.2 & $26 / 26$ & 100.0 & $23 / 26$ & 88,5 & $25 / 26$ & 96.2 & $22 / 26$ & 84.6 \\
\hline & $=0.180 \%$ & $16 / 18$ & 88.9 & $15 / 18$ & 83.3 & $17 / 18$ & 94.4 & $13 / 18$ & 72.2 & $16 / 18$ & 88.9 & $13 / 18$ & 72.2 \\
\hline \multirow{7}{*}{$\begin{array}{l}\text { AGE } \\
=30\end{array}$} & $<0.030 \%$ & $6 / 16$ & 37.5 & $2 / 16$ & 12.5 & $5 / 16$ & 31.3 & $2 / 16$ & 12.5 & $2 / 16$ & 12.5 & $1 / 16$ & 6.3 \\
\hline & $0.030-0.059 \%$ & $3 / 10$ & 30.0 & $1 / 10$ & 10.0 & $3 / 10$ & 30.0 & $1 / 10$ & 10.0 & $3 / 10$ & 30.0 & $1 / 10$ & 10.0 \\
\hline & $0.060-0.089 \%$ & $1 / 5$ & 20.0 & $1 / 5$ & 20.0 & $2 / 5$ & 40.0 & $0 / 5$ & 0.0 & $0 / 5$ & 0.0 & $0 / 5$ & 0,0 \\
\hline & $0.090-0.119 \%$ & $5 / 10$ & 50.0 & $5 / 10$ & 50.0 & $7 / 10$ & 70.0 & $5 / 10$ & 50.0 & $7 / 10$ & 70.0 & $5 / 10$ & 50,0 \\
\hline & $0.120-0.149 \%$ & $8 / 12$ & 66.7 & $8 / 12$ & 66,7 & $9 / 12$ & 75.0 & $7 / 12$ & 58.3 & $8 / 12$ & 66.7 & $7 / 12$ & 58.3 \\
\hline & $0.150-0.179 \%$ & $12 / 16$ & 75.0 & $12 / 16$ & 75.0 & $16 / 16$ & 100.0 & $11 / 16$ & 68.8 & $15 / 16$ & 93.8 & $11 / 16$ & 68.8 \\
\hline & $=0.180 \%$ & $8 / 10$ & 80.0 & $8 / 10$ & 80.0 & $9 / 10$ & 90.0 & $8 / 10$ & 80.0 & $9 / 10$ & 90.0 & $8 / 10$ & 80.0 \\
\hline
\end{tabular}


STRATEGY

\begin{tabular}{|c|c|c|c|c|c|c|c|c|c|c|c|c|}
\hline $\begin{array}{l}\text { SUBJECT } \\
\text { CATEGORY }\end{array}$ & $\begin{array}{l}\text { BAC C LASS } \\
\text { INTER VAL }\end{array}$ & \multicolumn{2}{|c|}{$1-12$ th $\%$} & \multicolumn{2}{|c|}{$\mathrm{N}^{1-16 \mathrm{th}}$} & \multicolumn{2}{|c|}{$N^{2-16 t h}$} & $\mathrm{~N}^{1-20 \mathrm{th}} \%$ & \multicolumn{2}{|c|}{$1-24$ th $\%$} & \multicolumn{2}{|c|}{$2-24 t h$} \\
\hline \multirow{7}{*}{$\begin{array}{l}\text { ALL } \\
\text { SUBJECTS }\end{array}$} & $<0.030 \%$ & $9 / 59$ & 15.3 & $5 / 59$ & 8.5 & $14 / 59$ & 23.7 & $5 / 59$ & $5 / 59$ & 8.5 & $10 / 59$ & 16.9 \\
\hline & $0.030-0.059 \%$ & $4 / 24$ & 16.7 & $4 / 24$ & 16.7 & $9 / 24$ & 37,5 & $4 / 24 \quad 16.7$ & $3 / 24$ & 12.5 & $6 / 24$ & 25,0 \\
\hline & $0.060-0.089 \%$ & $5 / 19$ & 26.3 & $4 / 19$ & 21.1 & $6 / 19$ & 31.6 & $4 / 19 \quad 21.1$ & $3 / 19$ & 15.8 & $5 / 19$ & 26.3 \\
\hline & $0.090-0.119 \%$ & $15 / 36$ & 41.7 & $15 / 36$ & 41.7 & $16 / 36$ & 44.4 & $14 / 36 \quad 38.9$ & $12 / 36$ & 33.3 & $15 / 36$ & 41.7 \\
\hline & $0.120-0.149 \%$ & $24 / 49$ & 49.0 & $19 / 49$ & 38.8 & $27 / 49$ & 55.1 & $18 / 49 \quad 36.7$ & $18 / 49$ & 36.7 & $24 / 49$ & 49.0 \\
\hline & $0.150-0.179 \%$ & $32 / 52$ & 61.5 & $31 / 52$ & 59.6 & $42 / 52$ & 80.8 & $28 / 52 \quad 53.8$ & $29 / 52$ & 55.8 & $34 / 52$ & 65.4 \\
\hline & $=0.180 \%$ & $13 / 21$ & 61.9 & $13 / 21$ & 61.9 & $18 / 21$ & 85.7 & $11 / 21$ & $11 / 21$ & 52.4 & $16 / 21$ & 76.2 \\
\hline \multirow{7}{*}{ MALES } & $=0.030 \%$ & $5 / 36$ & 13.9 & $4 / 36$ & 11.1 & $9 / 36$ & 25.0 & $4 / 36$ & $4 / 36$ & 11.1 & $6 / 36$ & 16.7 \\
\hline & $0.030-0.059 \%$ & $3 / 17$ & 17.6 & $3 / 17$ & 17.6 & $7 / 17$ & 41.2 & $4 / 17$ & $3 / 17$ & 17.6 & $6 / 17$ & 35.3 \\
\hline & $0.060-0.089 \%$ & $3 / 12$ & 25.0 & $3 / 12$ & 25.0 & $4 / 12$ & 33.3 & $3 / 12 \quad 25,0$ & $3 / 12$ & 25.0 & $4 / 12$ & 33.3 \\
\hline & $0.090-0.119 \%$ & $12 / 28$ & 42.9 & $12 / 28$ & 42.9 & $13 / 28$ & 46.4 & $11 / 28 \quad 39.3$ & $11 / 28$ & 39.3 & $12 / 28$ & 42.9 \\
\hline & $0.120-0.149 \%$ & $17 / 34$ & 50.0 & $15 / 34$ & 44. 1 & $20 / 34$ & 58.8 & $14 / 34 \quad 41,2$ & $14 / 34$ & 41.2 & $18 / 34$ & 52.9 \\
\hline & $0.150-0.179 \%$ & $16 / 30$ & 53.3 & $14 / 30$ & 46.7 & $22 / 30$ & 73.3 & $11 / 30 \quad 36.7$ & $12 / 30$ & 40.0 & $14 / 30$ & 46.7 \\
\hline & $=0.180 \%$ & $6 / 11$ & 54.5 & $6 / 11$ & 54.5 & $9 / 11$ & 81.8 & $4 / 11$ & $4 / 11$ & 36.4 & $7 / 11$ & 63.6 \\
\hline \multirow{7}{*}{ FEMALES } & $=0.030 \%$ & $4 / 23$ & 17.4 & $1 / 23$ & 4.3 & $5 / 23$ & 21.7 & $1 / 23$ & $1 / 23$ & 4.3 & $4 / 23$ & 17.4 \\
\hline & $0.030-0.059 \%$ & $1 / 7$ & 14.3 & $1 / 7$ & 14.3 & $2 / 7$ & 28.6 & 0.0 & $0 / 7$ & 0.0 & $0 / 7$ & 0.0 \\
\hline & $0.060-0.089 \%$ & $2 / 7$ & 28.6 & $1 / 7$ & 14.3 & $2 / 7$ & 28.6 & 14.3 & $0 / 7$ & 0.0 & $1 / 7$ & 14.3 \\
\hline & $0.090-0.119 \%$ & $3 / 8$ & 37.5 & $3 / 8$ & 37.5 & $3 / 8$ & 37.5 & 37.5 & $1 / 8$ & 12.5 & $3 / 8$ & 37.5 \\
\hline & $0.120-0.149 \%$ & $7 / 15$ & 46.7 & $4 / 15$ & 26.7 & $7 / 15$ & 46.7 & 26.7 & $4 / 15$ & 26.7 & $6 / 15$ & 40.0 \\
\hline & $0.150-0.179 \%$ & $16 / 22$ & 72.7 & $17 / 22$ & 77.3 & $20 / 22$ & 90.9 & $17 / 22,77,3$ & $17 / 22$ & 77.3 & $20 / 22$ & 90.9 \\
\hline & $=0.180 \%$ & $7 / 10$ & 70.0 & $7 / 10$ & 70.0 & $9 / 10$ & 90.0 & 70.0 & $7 / 10$ & 70.0 & $9 / 10$ & 90.0 \\
\hline \multirow{7}{*}{$\begin{array}{l}\text { AGE } \\
<30\end{array}$} & $<0.030 \%$ & $4 / 25$ & 16.0 & $3 / 25$ & 12.0 & $5 / 25$ & 20.0 & $3 / 25$ & $3 / 25$ & 12.0 & $3 / 25$ & 12.0 \\
\hline & $0.030-0.059 \%$ & $2 / 14$ & 14.3 & $2 / 14$ & 14.3 & $5 / 14$ & 35.7 & $2 / 14$ & $1 / 14$ & 7.1 & $3 / 14$ & $21: 4$ \\
\hline & $0.060-0.089 \%$ & $2 / 7$ & 28.6 & $2 / 7$ & 28.6 & $2 / 7$ & 28.6 & 28.6 & $1 / 7$ & 14.3 & $1 / 7$ & 14.3 \\
\hline & $0.090-0.119 \%$ & $5 / 13$ & 38.5 & $5 / 13$ & 38.5 & $5 / 13$ & 38.5 & $5 / 13$ & $4 / 13$ & 30.8 & $5 / 13$ & 38.5 \\
\hline & $0.120-0.149 \%$ & $13 / 29$ & 44.8 & $10 / 29$ & 34.5 & $15 / 29$ & 51.7 & $10 / 29 \quad 34,5$ & $10 / 29$ & 34.5 & $14 / 29$ & 48.3 \\
\hline & $0.150-0.179 \%$ & $15 / 23$ & 65.2 & $14 / 23$ & 60.9 & $20 / 23$ & 87.0 & $14 / 23 \quad 60.9$ & $15 / 23$ & 65.2 & $17 / 23$ & 73.9 \\
\hline & $=0.180 \%$ & $6 / 9$ & 66.7 & $6 / 9$ & 66.7 & $7 / 9$ & 66.7 & $6 / 9 \quad 66.7$ & $6 / 9$ & 66.7 & $6 / 9$ & 66.7 \\
\hline \multirow{7}{*}{$\begin{array}{l}\text { AGE } \\
\geq 30\end{array}$} & $=0.030 \%$ & $5 / 34$ & 14.7 & $2 / 34$ & 5.9 & $9 / 34$ & 26.5 & $2 / 34$ & $2 / 34$ & 5.9 & $7 / 34$ & 20.6 \\
\hline & $0.030-0.059 \%$ & $2 / 10$ & 20.0 & $2 / 10$ & 20.0 & $4 / 10$ & 40.0 & $2 / 10$ & $2 / 10$ & 20.0 & $3 / 10$ & 30.0 \\
\hline & $0.060-0.089 \%$ & $3 / 12$ & 25.0 & $2 / 12$ & 16.7 & $4 / 12$ & 33.3 & $2 / 12$ & $2 / 12$ & 16.7 & $4 / 12$ & 33.3 \\
\hline & $0.090-0.119 \%$ & $10 / 23$ & 43.5 & $10 / 23$ & 43.5 & $11 / 23$ & 47.8 & $9 / 23$ & $8 / 23$ & 34.8 & $10 / 23$ & 43.5 \\
\hline & $0.120-0.149 \%$ & $11 / 20$ & 55.0 & $9 / 20$ & 45.0 & $12 / 20$ & 60.0 & $8 / 20$ & $8 / 20$ & 40.0 & $10 / 20$ & 50.0 \\
\hline & $0.150-0.179 \%$ & $17 / 29$ & 58.6 & $17 / 29$ & 58.6 & $22 / 29$ & 75,9 & $14 / 29 \quad 48,3$ & $14 / 29$ & 48.3 & $17 / 29$ & 58.6 \\
\hline & $=0.180 \%$ & $7 / 12$ & 58.3 & $7 / 12$ & 58.3 & $11 / 12$ & 91.7 & $5 / 12 \quad 41.7$ & $5 / 12$ & 41.7 & $10 / 12$ & 83.3 \\
\hline
\end{tabular}


Table XVII

Quickey Control Session Performance, Group III

STRATEGY

\begin{tabular}{|c|c|c|c|c|c|c|c|c|c|c|c|c|c|c|}
\hline \multirow{2}{*}{$\begin{array}{l}\text { SUB JECT } \\
\text { CATEGORY }\end{array}$} & \multirow{2}{*}{$\begin{array}{c}\text { TEST } \\
i\end{array}$} & \multirow{2}{*}{$\begin{array}{l}\text { CYCLE } \\
\text { TIME* } \\
\end{array}$} & \multicolumn{2}{|c|}{$1-12$ th } & \multicolumn{2}{|c|}{$1-16$ th } & \multicolumn{2}{|c|}{$2-16 \mathrm{th}$} & \multicolumn{2}{|c|}{$1-20$ th } & \multicolumn{2}{|c|}{$1-24$ th } & \multicolumn{2}{|c|}{ 2-24th } \\
\hline & & & $\mathbf{N}$ & $\%$ & $\bar{N}$ & \% & $\mathbf{N}$ & $\overline{\%}$ & $\mathbf{N}$ & $\%$ & & $\%$ & & $\%$ \\
\hline \multirow{9}{*}{$\begin{array}{l}\text { ALL } \\
\text { SUBJECTS }\end{array}$} & 1. & $0: 10$ & $6 / 14$ & 42.9 & $6 / 14$ & 42.9 & $7 / 14$ & 50.0 & $5 / 14$ & 35. : & $3 / 14$ & 21.4 & $5 / 14$ & 35.7 \\
\hline & 2. & $0: 35$ & $6 / 14$ & 42.9 & $4 / 14$ & 28.6 & $6 / 14$ & 42.9 & $4 / 14$ & 28.6 & $1 / 14$ & 7.1 & $5 / 14$ & 35.7 \\
\hline & 3. & $1: 35$ & $3 / 14$ & 21.4 & $2 / 14$ & 14.3 & $3 / 14$ & 21.4 & $1 / 14$ & 7. 11 & $1 / 14$ & 7.1 & $2 / 14$ & 14.3 \\
\hline & 4. & $2: 35$ & $1 / 14$ & 7.1 & $0 / 14$ & 0.0 & $3 / 14$ & 21.4 & $0 / 14$ & 0.0 & $0 / 14$ & 0.0 & $0 / 14$ & 0.0 \\
\hline & 5. & $3: 35$ & $1 / 14$ & 7.1 & $1 / 14$ & 7.1 & $3 / 14$ & 21.4 & $1 / 14$ & 7.: 1 & $1 / 14$ & 7.1 & $2 / 14$ & 14.3 \\
\hline & 6. & $4: 35$ & $3 / 14$ & 21.4 & $3 / 14$ & 21.4 & $3 / 14$ & 21.4 & $1 / 14$ & 7.:1 & $1 / 14$ & 7.1 & $1 / 14$ & 7.1 \\
\hline & 7. & 5:00 & $2 / 14$ & 14.3 & $1 / 14$ & 7.1 & $4 / 14$ & 14.3 & $1 / 14$ & 7. 1 & $1 / 14$ & 7.1 & $2 / 14$ & 14.3 \\
\hline & 8. & $6: 00$ & $3 / 14$ & 21.4 & $1 / 14$ & 7.1 & $4 / 14$ & 14.3 & $1 / 14$ & 7. 1 & $0 / 14$ & 0.0 & $3 / 14$ & 21.4 \\
\hline & 9. & $6: 30$ & $2 / 14$ & 14.3 & $2 / 14$ & 14.3 & $3 / 14$ & 21.4 & $1 / 14$ & 7. 1 & $1 / 14$ & 7.1 & $1 / 14$ & 7.1 \\
\hline \multirow{9}{*}{ MALES } & 1. & $0: 10$ & $3 / 8$ & 37.5 & $3 / 8$ & 37.5 & $3 / 8$ & 37.5 & $2 / 8$ & 25.0 & $2 / 8$ & 25.0 & $2 / 8$ & 25.0 \\
\hline & 2. & $0: 35$ & $3 / 8$ & 37.5 & $2 / 8$ & 25.0 & $3 / 8$ & 37.5 & $2 / 8$ & $25.1)$ & $1 / 8$ & 12.5 & $3 / 8$ & 37.5 \\
\hline & 3. & $\mathrm{I}: 35$ & $2 / 8$ & 25.0 & $1 / 8$ & 12.5 & $2 / 8$ & 25.0 & $0 / 8$ & $0.1)$ & $0 / 8$ & 0.0 & $1 / 8$ & 12.5 \\
\hline & 4. & $2: 35$ & $1 / 8$ & 12.5 & $0 / 8$ & 0.0 & $2 / 8$ & 25.0 & $0 / 8$ & 0.0 & $10 / 8$ & 0.0 & $0 / 8$ & 0.0 \\
\hline & 5. & $3: 35$ & $1 / 8$ & 12.5 & $1 / 8$ & 12.5 & $3 / 8$ & 37.5 & $1 / 8$ & 12.5 & $1 / 8$ & 12.5 & $2 / 8$ & 25.0 \\
\hline & 6. & $4: 35$ & $2 / 8$ & 25.0 & $2 / 8$ & 25.0 & $2 / 8$ & 25.0 & $1 / 8$ & 12.5 & $1 / 8$ & 12.5 & $1 / 8$ & 12.5 \\
\hline & 7. & $5: 00$ & $1 / 8$ & 12.5 & $1 / 8$ & 12.5 & $2 / 8$ & 25.0 & $1 / 8$ & 12.5 & $1 / 8$ & 12.5 & $1 / 8$ & 12.5 \\
\hline & 8. & 6:00 & $1 / 8$ & 12.5 & $0 / 8$ & 0.0 & $2 / 8$ & 25.0 & $0 / 8$ & 0.0 & $0 / 8$ & 0.0 & $1 / 8$ & 12.5 \\
\hline & 9. & $6: 30$ & $2 / 8$ & 25.0 & $2 / 8$ & 25.0 & $2 / 8$ & 25.0 & $1 / 8$ & 12.5 & $1 / 8$ & 12.5 & $1 / 8$ & 12.5 \\
\hline \multirow{9}{*}{ FEMALES } & 1. & $0: 10$ & $3 / 6$ & 50.0 & $3 / 6$ & 50.0 & $4 / 6$ & 66.7 & $3 / 6$ & 50.0 & $1 / 6$ & 16.7 & $3 / 6$ & 50.0 \\
\hline & 2. & $0: 35$ & $3 / 6$ & 50.0 & $2 / 6$ & 33.3 & $3 / 6$ & 50.0 & $2 / 6$ & 33.3 & $0 / 6$ & 0.0 & $2 / 6$ & 33.3 \\
\hline & 3. & $1: 35$ & $1 / 6$ & 16.7 & $1 / 6$ & 16.7 & $1 / 6$ & 16.7 & $1 / 6$ & 16.7 & $1 / 6$ & 16.7 & $1 / 6$ & 16.7 \\
\hline & 4. & $2: 35$ & $0 / 6$ & 0.0 & $0 / 6$ & 0.0 & $1 / 6$ & 16.7 & $0 / 6$ & 0.0 & $0 / 6$ & 0.0 & $0 / 6$ & 0.0 \\
\hline & 5. & $3: 35$ & $0 / 6$ & 0.0 & $0 / 6$ & 0.0 & $0 / 6$ & 0.0 & $0 / 6$ & 0.0 & $0 / 6$ & 0.0 & $0 / 6$ & 0.0 \\
\hline & 6. & $4: 35$ & $1 / 6$ & 16.7 & $1 / 6$ & 16.7 & 1.6 & 16.7 & $0 / 6$ & 0.0 & $0 / 6$ & 0.0 & $0 / 6$ & 0.0 \\
\hline & 7. & 5:00 & $1 / 6$ & 16.7 & $0 / 6$ & 0.0 & $2 / 6$ & 33.3 & $0 / 6$ & 0.0 & $0 / 6$ & 0.0 & $1 / 6$ & 16.7 \\
\hline & 8. & $6: 00$ & $2 / 6$ & 33.3 & $1 / 6$ & 16.7 & $2 / 6$ & 33.3 & $1 / 6$ & 16.7 & $0 / 6$ & 0.0 & $2 / 6$ & 33,3 \\
\hline & 9. & $6: 30$ & $0 / 6$ & 0.0 & $0 / 6$ & 0.0 & $1 / 6$ & 16.7 & $0 / 6$ & 0.0 & $0 / 6$ & 0.0 & $0 / 6$ & 0.0 \\
\hline \multirow{9}{*}{$\mathrm{AGE}<30$} & 1. & $0: 10$ & $3 / 6$ & 50.0 & $3 / 6$ & 50,0 & $3 / 6$ & 50,0 & $2 / 6$ & 33,3 & $1 / 6$ & 16.7 & $1 / 6$ & 16.7 \\
\hline & 2. & $0: 35$ & $3 / 6$ & 50.0 & $2 / 6$ & 33,3 & $3 / 6$ & 50.0 & $2 / 6$ & 33.3 & $0 / 6$ & 0,0 & $3 / 6$ & 50.0 \\
\hline & 3. & $1: 35$ & $2 / 6$ & 33.3 & $1 / 6$ & 16.7 & $2 / 6$ & 33,3 & $1 / 6$ & 16.7 & $1 / 6$ & 16.7 & $2 / 6$ & 33.3 \\
\hline & 4. & $2: 35$ & $1 / 6$ & 16.7 & $0 / 6$ & 0.0 & $2 / 6$ & 33,3 & $0 / 6$ & 0.0 & $0 / 6$ & 0,0 & $0 / 6$ & 0.0 \\
\hline & 5. & $3: 35$ & $1 / 6$ & 16.7 & $1 / 6$ & 16.7 & $1 / 6$ & 16.7 & $1 / 6$ & 16.7 & $1 / 6$ & 16.7 & $1 / 6$ & 16.7 \\
\hline & 6. & $4: 35$ & $1 / 6$ & 16.7 & $1 / 6$ & 16.7 & $1 / 6$ & 16,7 & $0 / 6$ & 0.0 & $0 / 6$ & 0.0 & $0 / 6$ & 0.0 \\
\hline & 7. & $5: 00$ & $1 / 6$ & 16.7 & $1 / 6$ & 16.7 & $2 / 6$ & 33.3 & $1 / 6$ & 16.7 & $1 / 6$ & 16.7 & 216 & 33.3 \\
\hline & 8. & $6: 00$ & $3 / 6$ & 50.0 & $1 / 6$ & 16.7 & $3 / 6$ & 50.0 & $1 / 6$ & $16 . i$ & $0 / 6$ & 0.0 & $3 / 6$ & 50.0 \\
\hline & 9. & $6: 30$ & $1 / 6$ & 16.7 & $1 / 6$ & 16.7 & $2 / 6$ & 33.3 & $1 / 6$ & 16.7 & $1 / 6$ & 16.7 & $1 / 6$ & 16.7 \\
\hline \multirow{9}{*}{$A G E \geq 30$} & 1. & $0: 10$ & $3 / 8$ & 37.5 & $3 / 8$ & 37.5 & $4 / 8$ & 50.0 & $3 / 8$ & 37.5 & $2 / 8$ & 25.0 & $4 / 8$ & 50.0 \\
\hline & 2. & $0: 35$ & $3 / 8$ & 37.5 & $2 / 8$ & 25.0 & $3 / 8$ & 37.5 & $2 / 8$ & 25.0 & $1 / 8$ & 12.5 & $2 / 8$ & 25.0 \\
\hline & 3. & $1: 35$ & $1 / 8$ & 12.5 & $1 / 8$ & 12.5 & $1 / 8$ & 12.5 & $0 / 8$ & 0.0 & $0 / 8$ & 0.0 & $0 / 8$ & 0.0 \\
\hline & 4. & $2: 35$ & $0 / 8$ & 0.0 & $0 / 8$ & 0.0 & $1 / 8$ & 12.5 & $0 / 8$ & 0.0 & $0 / 8$ & 9.0 & $0 / 8$ & 0.0 \\
\hline & 5. & $3: 35$ & $0 / 8$ & 0.0 & $0 / 8$ & 0.0 & $2 / 8$ & 25.0 & $0 / 8$ & 0,0 & $0 / 8$ & 0.0 & $1 / 8$ & 12.5 \\
\hline & 6. & $4: 35$ & $2 / 8$ & 25.0 & $2 / 8$ & 25.0 & $2 / 8$ & 25.0 & $1 / 8$ & 12. 5 & $1 / 8$ & 12.5 & $1 / 8$ & 12.5 \\
\hline & 7. & $5: 00$ & $1 / 8$ & 12.5 & $0 / 8$ & 0.0 & $2 / 8$ & 25.0 & $0 / 8$ & 0.0 & $0 / 8$ & 0.0 & $0 / 8$ & 0.0 \\
\hline & 8. & 6:00 & $0 / 8$ & 0.0 & $0 / 8$ & 0.0 & $1 / 8$ & 12.5 & $0 / 8$ & 0.0 & $0 / 8$ & 0.0 & $0 / 8$ & 0.0 \\
\hline & 9. & $6: 30$ & $1 / 8$ & 12.5 & $1 / 8$ & 12,5 & $1 / 8$ & 12,5 & $0 / 8$ & 0.0 & $10 / 8$ & 0.0 & $10 / 8$ & 0.0 \\
\hline
\end{tabular}


Table XVIII

Quickey Performance with Adjusted Criterion

\begin{tabular}{|c|c|c|c|c|c|c|c|c|}
\hline \multirow{3}{*}{$\begin{array}{r}\text { BAC } \\
<0.030\end{array}$} & \multicolumn{4}{|c|}{ Group II } & \multicolumn{4}{|c|}{ Group III } \\
\hline & \multicolumn{2}{|c|}{$\begin{array}{l}\text { At Least } 1 \\
\text { Criterion } \\
\text { Response }\end{array}$} & \multicolumn{2}{|c|}{$\begin{array}{l}\text { At Least } 2 \\
\text { Criterion } \\
\text { Responses }\end{array}$} & \multicolumn{2}{|c|}{$\begin{array}{l}\text { At Least } 1 \\
\text { Criterion } \\
\text { Response } \\
\end{array}$} & \multicolumn{2}{|c|}{$\begin{array}{l}\text { At Ieast } 2 \\
\text { Criterion } \\
\text { Responses }\end{array}$} \\
\hline & $0 / 46$ & $0.00 \%$ & $0 / 46$ & $0.00 \%$ & $2 / 59$ & $3.4 \%$ & $2 / 59$ & $3.4 \%$ \\
\hline $\begin{array}{r}.030 \\
.059\end{array}$ & $1 / 32$ & $3.1 \%$ & $2 / 32$ & $6.3 \%$ & $1 / 24$ & $4.2 \%$ & $2 / 24$ & $8.3 \%$ \\
\hline $\begin{array}{l}.060 \\
.089\end{array}$ & $0 / 16$ & $0.00 \%$ & $3 / 13$ & $23.1 \%$ & $2 / 19$ & $10.5 \%$ & $2 / 19$ & $10.5 \%$ \\
\hline $\begin{array}{r}.090 \\
.119\end{array}$ & $13 / 36$ & $36.1 \%$ & $23 / 36$ & $63.9 \%$ & $6 / 36$ & $16.7 \%$ & $9 / 36$ & $25.0 \%$ \\
\hline $\begin{array}{l}.120 \\
.149\end{array}$ & $20 / 35$ & $57.1 \%$ & $26 / 35$ & $74.3 \%$ & $13 / 49$ & $26.5 \%$ & $16 / 49$ & $32.7 \%$ \\
\hline $\begin{array}{l}.150 \\
.17 \theta\end{array}$ & $26 / 42$ & $61.9 \%$ & $34 / 42$ & $80.1 \%$ & $13 / 52$ & $25.0 \%$ & $19 / 52$ & $36.5 \%$ \\
\hline$\geq .180$ & $18 / 28$ & $64.3 \%$ & $22 / 28$ & $78.6 \%$ & $5 / 21$ & $23.8 \%$ & $12 / 21$ & $57.1 \%$ \\
\hline
\end{tabular}


Table XIX

Quickey Performance, Group IIIC

STRATEGY

\begin{tabular}{|c|c|c|c|c|c|c|c|c|c|c|c|c|c|}
\hline $\begin{array}{l}\text { SUBJECT } \\
\text { CATEGORY }\end{array}$ & $\begin{array}{l}\text { BAC C LASS } \\
\text { INTER VAL }\end{array}$ & \multicolumn{2}{|c|}{$\mathrm{N}^{1-12 \text { th }} \%$} & \multicolumn{2}{|c|}{$N^{1-16 \text { th }}$} & \multicolumn{2}{|c|}{$\mathrm{N}^{2-16 \mathrm{th}} \%$} & \multicolumn{2}{|c|}{$\mathrm{N}^{1-20 \text { th }} \%$} & \multicolumn{2}{|c|}{$\mathrm{N}^{1-24 \mathrm{th}}$} & \multicolumn{2}{|c|}{$2-24$ th } \\
\hline \multirow{7}{*}{$\begin{array}{l}\text { ALL } \\
\text { SUBJECTS }\end{array}$} & $<0.030 \%$ & $0 / 22$ & 0.0 & $0 / 22$ & 0.0 & $0 / 22$ & 0.0 & $0 / 22$ & 0.0 & $0 / 22$ & 0.0 & $0 / 22$ & 0.0 \\
\hline & $0: 030-0.059 \%$ & $0 / 8$ & 0.0 & $0 / 8$ & 0.0 & $0 / 8$ & .0 .0 & $0 / 8$ & 0.0 & $0 / 8$ & 0.0 & $0 / 8$ & 0.0 \\
\hline & $0.060-0.089 \%$ & $0 / 6$ & 0.0 & $0 / 6$ & 0.0 & $0 / 6$ & 0.0 & $0 / 6$ & 0.0 & $0 / 6$ & 0.0 & 0.6 & 0.0 \\
\hline & $0.090-0.119 \%$ & $0 / 10$ & 0.0 & $0 / 10$ & 0.0 & $1 / 10$ & 10.0 & $0 / 10$ & 0.0 & $0 / 10$ & 0.0 & $0 / 10$ & 0.0 \\
\hline & $0.120-0.149 \%$ & $0 / 16$ & 0.0 & $0 / 16$ & 0.0 & $0 / 16$ & 0.0 & $0 / 16$ & 0.0 & $1 / 16$ & 6.3 & $1 / 16$ & 6.3 \\
\hline & $0.150-0.179 \%$ & $1 / 22$ & 4.5 & $1 / 22$ & 4.5 & $3 / 22$ & 13.6 & $1 / 22$ & 4.5 & $0 / 22$ & 0.0 & $0 / 22$ & 0.0 \\
\hline & $\geq 0.180 \%$ & $1 / 12$ & 8.3 & $1 / 12$ & 8.3 & $2 / 12$ & 16.7 & $0 / 12$ & 0.0 & $0 / 12$ & 0.0 & $1 / 12$ & 8.3 \\
\hline \multirow{7}{*}{ MALES } & $<0.030 \%$ & $0 / 6$ & 0.0 & $0 / 6$ & 0.0 & $0 / 6$ & 0.0 & $0 / 6$ & 0.0 & $0 / 6$ & 0.0 & $0 / 6$ & 0.0 \\
\hline & $0.030-0.059 \%$ & $0 / 2$ & 0.0 & $0 / 2$ & 0.0 & $0 / 2$ & 0.0 & $0 / 2$ & 0.0 & $0 / 2$ & 0.0 & $0 / 2$ & 0.0 \\
\hline & $0.060-0.089 \%$ & $0 / 2$ & 0.0 & $\theta / 2$ & 0.0 & $0 / 2$ & 0.0 & $0 / 2$ & 0.0 & $0 / 2$ & 0.0 & $0 / 2$ & 0.0 \\
\hline & $0.090-0.119 \%$ & $0 / 4$ & 0.0 & $0 / 4$ & 0.0 & $0 / 4$ & 0.0 & $0 / 4$ & 0.0 & $0 / 4$ & 0.0 & $0 / 4$ & 0.0 \\
\hline & $0.120-0.149 \%$ & - & & & & & & - & & - & & & \\
\hline & $0.150-0.179 \%$ & $0 / 8$ & 0.0 & $0 / 8$ & 0.0 & $0 / 8$ & 0.0 & $0 / 8$ & 0.0 & $0 / 8$ & 0.0 & $0 / 8$ & 0.0 \\
\hline & $=0.180 \%$ & $1 / 10$ & 10.0 & $1 / 19$ & 10.0 & $2 / 10$ & 20.0 & $0 / 10$ & 0.0 & $0 / 10$ & 0.0 & $1 / 10$ & 10.0 \\
\hline \multirow{7}{*}{ FEMALES } & $<0.030 \%$ & $0 / 16$ & 0.0 & $0 / 16$ & 0.0 & $0 / 16$ & 0.0 & $0 / 16$ & 0.0 & $0 / 16$ & 0.0 & $0 / 16$ & 0.0 \\
\hline & $0.030-0.059 \%$ & $0 / 6$ & 0.0 & $0 / 6$ & 0.0 & $0 / 6$ & 0.0 & $0 / 6$ & 0.0 & $0 / 6$ & 0.0 & $0 / 6$ & 0.0 \\
\hline & $0.060-0.089 \%$ & $0 / 4$ & 0.0 & $0 / 4$ & 0.0 & $0 / 4$ & 0.0 & $0 / 4$ & 0.0 & $0 / 4$ & 0.0 & $0 / 4$ & 0.0 \\
\hline & $0.090-0.119 \%$ & $0 / 6$ & 0.0 & $0 / 6$ & 0.0 & $1 / 6$ & 16.7 & $0 / 6$ & 0.0 & $0 / 6$ & 0.0 & $0 / 6$ & 0.0 \\
\hline & $0.120-0.149 \%$ & $0 / 16$ & 0.0 & $0 / 16$ & 0.0 & $0 / 16$ & 0.0 & $0 / 16$ & 0.0 & $1 / 16$ & 6.3 & $1 / 16$ & 6.3 \\
\hline & $0.150-0.179 \%$ & $1 / 14$ & 7.1 & $1 / 14$ & 7.1 & $3 / 14$ & 21.4 & $1 / 14$ & 7.1 & $0 / 14$ & 0.0 & $0 / 14$ & 0.0 \\
\hline & $=0.180 \%$ & $0 / 2$ & 0.0 & $0 / 2$ & 0.0 & $0 / 2$ & 0.0 & $0 / 2$ & 0.0 & $0 / 2$ & 0.0 & $0 / 2$ & 0.0 \\
\hline \multirow{7}{*}{$\begin{array}{l}\text { AGE } \\
<30\end{array}$} & $=0.030 \%$ & $0 / 10$ & 0.0 & $0 / 10$ & 0.0 & $0 / 10$ & 0.0 & $0 / 10$ & 0.0 & $0 / 10$ & 0.0 & $0 / 10$ & 0.0 \\
\hline & $0.030-0.059 \%$ & $0 / 2$ & 0.0 & $0 / 2$ & $0.0 \mathrm{c}$ & $0 / 2$ & 0.0 & $0 / 2$ & 0.0 & $0 / 2$ & 0.0 & $0 / 2$ & 0.0 \\
\hline & $0.060-0.089 \%$ & $0 / 2$ & 0.0 & $0 / 2$ & 0.0 & $0 / 2$ & 0.0 & $0 / 2$ & 0.0 & $0 / 2$ & 0.0 & $0 / 2$ & 0.0 \\
\hline & $0.090-0.119 \%$ & $0 / 2$ & 0.0 & $0 / 2$ & 0.0 & $0 / 2$ & 0.0 & $0 / 2$ & 0.0 & $0 / 2$ & 0.0 & $0 / 2$ & 0.0 \\
\hline & $0.120-0.149 \%$ & $0 / 8$ & 0.0 & $0 / 8$ & 0.0 & $0 / 8$ & 0.0 & $0 / 8$ & 0.0 & $0 / 8$ & 0.0 & $0 / 8$ & 0.0 \\
\hline & $0.150-0.179 \%$ & $0 / 6$ & 0.0 & $0 / 6$ & 0.0 & $0 / 6$ & 0.0 & $0 / 6$ & 0.0 & $0 / 6$ & 0.0 & $0 / 6$ & 0.0 \\
\hline & $=0.180 \%$ & $0 / 2$ & 0.0 & $0 / 2$ & 0.0 & $0 / 2$ & 0.0 & $0 / 2$ & 0.0 & $0 / 2$ & 0.0 & $0 / 2$ & 0.0 \\
\hline \multirow{7}{*}{$\begin{array}{l}\text { AGE } \\
\geq 30\end{array}$} & $<0.030 \%$ & $0 / 12$ & 0.0 & $0 / 12$ & 0.0 & $0 / 12$ & 0.0 & $0 / 12$ & 0.0 & $0 / 12$ & 0.0 & $0 / 12$ & 0.0 \\
\hline & $0.030-0.059 \%$ & $0 / 6$ & 0.0 & $0 / 6$ & 0.0 & $0 / 6$ & 0.0 & $0 / 6$ & 0.0 & $0 / 6$ & 0.0 & $0 / 6$ & 0.0 \\
\hline & $0.060-0.089 \%$ & $0 / 4$ & 0.0 & $0 / 4$ & 0.0 & $0 / 4$ & 0.0 & $0 / 4$ & 0.0 & $0 / 4$ & 0.0 & $0 / 4$ & 0.0 \\
\hline & $0.090-0.119 \%$ & $0 / 8$ & 0.0 & $0 / 8$ & 0.0 & $1 / 8$ & 12.5 & $0 / 8$ & 0.0 & $0 / 8$ & 0.0 & $0 / 8$ & 0.0 \\
\hline & $0.120-0.149 \%$ & $0 / 8$ & 0.0 & $0 / 8$ & 0.0 & $0 / 8$ & 0.0 & $0 / 8$ & 0.0 & $1 / 8$ & 12.5 & $1 / 8$ & 12.5 \\
\hline & $0.150-0.179 \%$ & $1 / 16$ & 6.3 & $1 / 16$ & 6.3 & $3 / 16$ & 18.8 & $1 / 16$ & 6.3 & $0 / 16$ & 0.0 & $0 / 16$ & 0.0 \\
\hline & $=0.180 \%$ & $1 / 10$ & 10.0 & $1 / 10$ & 10.0 & $2 / 10$ & 20.0 & $0 / 10$ & 0.0 & $0 / 10$ & 0.0 & $1 / 10$ & 10.0 \\
\hline
\end{tabular}




\section{REACTION ANALYZER}

The Reaction Analyzer, developed by Raytheon, is based upon a tracking task of some 15 seconds duration. The Subject is required to turn a control knob in a clockwise direction through roughly $300^{\circ}$, bounded by positions labelled "START" and "FINISH," in such a manner as to keep two indicator lights (designated "SLOW" and "FAST") illuminated, ideally, with equal intensity. The turning rate required to maintain simultaneous illumination varies (essentially exponentially) as a function of elapsed time into trial.

Throughout the trial, the Subject accumulates error in proportion to the magnitude and duration of any difference in intensity of the two indicators. In order to pass, the cumulative error must not exceed a pre-set threshold. This threshold is controllable by means of a potentiometer mounted in the base of the instrument.

"PASS" and "FAIL" are displayed by indicator lights with those respective legends.

Training, testing, and analysis applied to the Reaction Analyzer are described below.

\section{A. Training Procedures}

The Reaction Analyzer was introduced into the program subsequent to the completion of Group I testing. Training conditions experienced by Groups II and III are described below.

Group II Subjects completed up to 5 blocks of 10 trials on each of their three training sessions. All trials took place at maximum degree of difficulty (i.e., minimum error threshold), corresponding to full counterclockwise position of the potentiometer. The Subject earned $\$ 5$ reward upon passing at least nine trials in a block. When this training criteria was achieved, no further trials were taken during that session. Subjects worked toward criterion and reward on each training session. Eight of the 12 Subjects achieved criterion on the first session, 10 on the second, and 11 on the third; the one Subject who did not achieve criterion on the third session had done so on both earlier sessions. Several Subjects were permitted more than the scheduled 50 trials in a given session when they were not able to reach criterion. The maximum number of trials on any one session was 70 , which occurred once. 
The minimum was 10 , which occurred 20 times during the 36 Subject-days of Group II training. The mean numbers of trials per Subject over the three training sessions were $41.7,15.0$, and 17.5 respectively, with an overall mean of 74.2 trials per Subject.

Examination of the results of Group II testing on the Reaction Analyzer (discussed in subsection $C$ below) indicated that the four older Subjects (age $>40$ ) had experienced considerable difficulty; they also required more extensive training (average of 107.5 trials) than had the eight younger (age $<30$ ) Subjects (average of 57.5 trials). A decision therefore was made to insure more thorough training for Group III. The se fifteen Subjects also completed up to 5 blocks of 10 trials on each of their three sessions. How ever, training commenced on the first session at a moderate degree of difficulty (potentiometer set midway between minimum and maximum error thresholds). Subjects were permitted to advance to the maximum degree of difficulty only after passing at least four of ten trials at the moderate setting. Thirteen Subjects did so on the first block, the remaining 2 on the second block. A more exacting criterion for $\$ 5$ reward was also implemented for Group III. This training criterion was 19 passes out of 20 trials in 2 consecutive blocks at maximum degree of difficulty. The absolute minimum number of trials on the first session was therefore 30 (since a.t least the first block took place at the moderate setting); 20 was the minimum number of the second and third sessions. The maximum number on any given session was 50 .

Nine of the Group III Subjects achieved criterion on the first session, 14 on the second, and all 15 on the third. All met criterion on at least two of the training sessions, and 8 did so on all three. The mean numbers of trials per Subject over the three sessions were 40.0, 23. 3, and 20.0 respectively, with an overall mean of 83.3 trials per Subject. Note that all 15 Subjects achieved training criterion on the third session in the minimum number of trials possible.

\section{B. Testing Procedures}

Reaction Analyzer testing for both Group II and Group III employed a block of five trials in each test cycle, with all trials taking place at maximum degree of difficulty. Thesecort recorded each trial as a "pass" or "fail." Group II Subjects were rewarded at the rate of $\$ 0.50$ for each trial passed. Group III Subjects received $\$ 0.25$ per pass with at $100 \%$ bonus if all five trials of the block were passed.

No equipment problems were noted for the Reaction Analyzer at any time. 


\section{Analyses and Results}

Analyses of Reaction Analyzer performance focused on the following strategies:

$$
\begin{aligned}
& \text { "one-out-of-one" } \\
& \text { "five-out-of-five" } \\
& \text { "four-out-of-five" } \\
& \text { "three-out-of-three" } \\
& \text { "two-out-of-three" }
\end{aligned}
$$

Results are presented in Tables XX and XXI for Groups II and III respectively. The third group's control session results are shown in Table XXII.

In comparing all Subjects of the two groups, it is evident that Group III experienced lower rejection rates than Group II (at times by a considerable margin) at all BACs and under all strategies. Analysis of variance under the $4 / 5$ strategy indicates that the difference between the groups is significant $(\mathrm{p}<.01)$. However, no significant difference is noted when only the younger Subjects (age $<30$ ) of the two groups are compared. It thus appears that procedures employed for Group II provided inadequate training for the four older Subjects (age -40$)$. There is, of course, no way of determining whether the Group III procedures would have corrected this problem for those individuals.

If data obtained from the older Group II Subjects are discounted, the Reaction Analyzer appears to offer promise under a number of different implementation schemes. Strategies such as $4 / 5$ or $2 / 3$ (depicted in Figures 13 and 14) conceivably could serve for future applications although the $30-40 \%$ rejection rates they would offer at high $\mathrm{BAC}$ are less than desired. Strategies like $3 / 3$ or $5 / 5$ (Figures 15 and 16) imply non-negligible rejection rates at zero $B A C$, but might nevertheless prove feasible.

Listed below are the results of analyses of variance of BAC versus sex and $B A C$ versus age, as applied to performance under the $4 / 5$ strategy. Neigher group evidenced significant difference with respect to sex; only Group II showed significant difference with respect to age. 


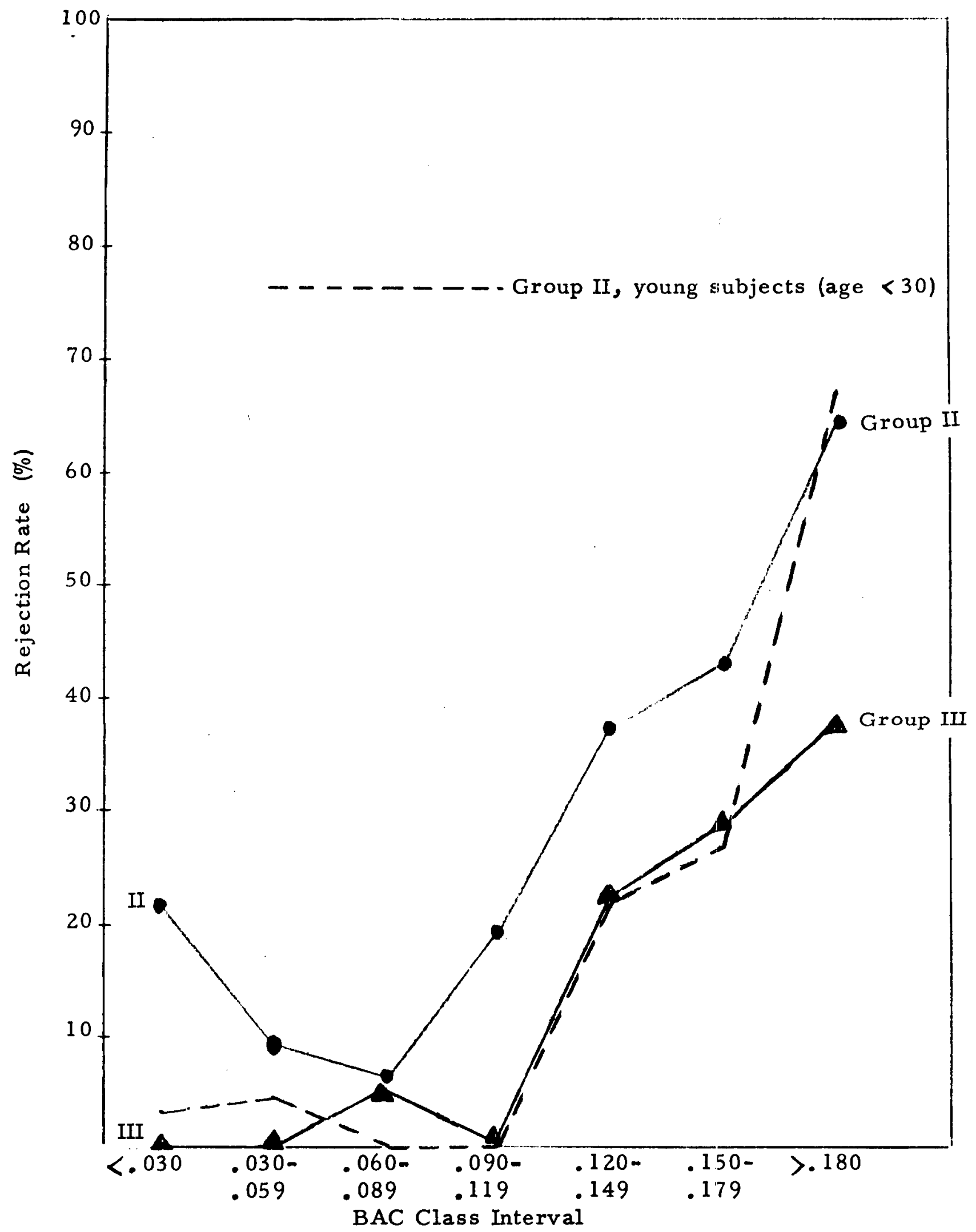




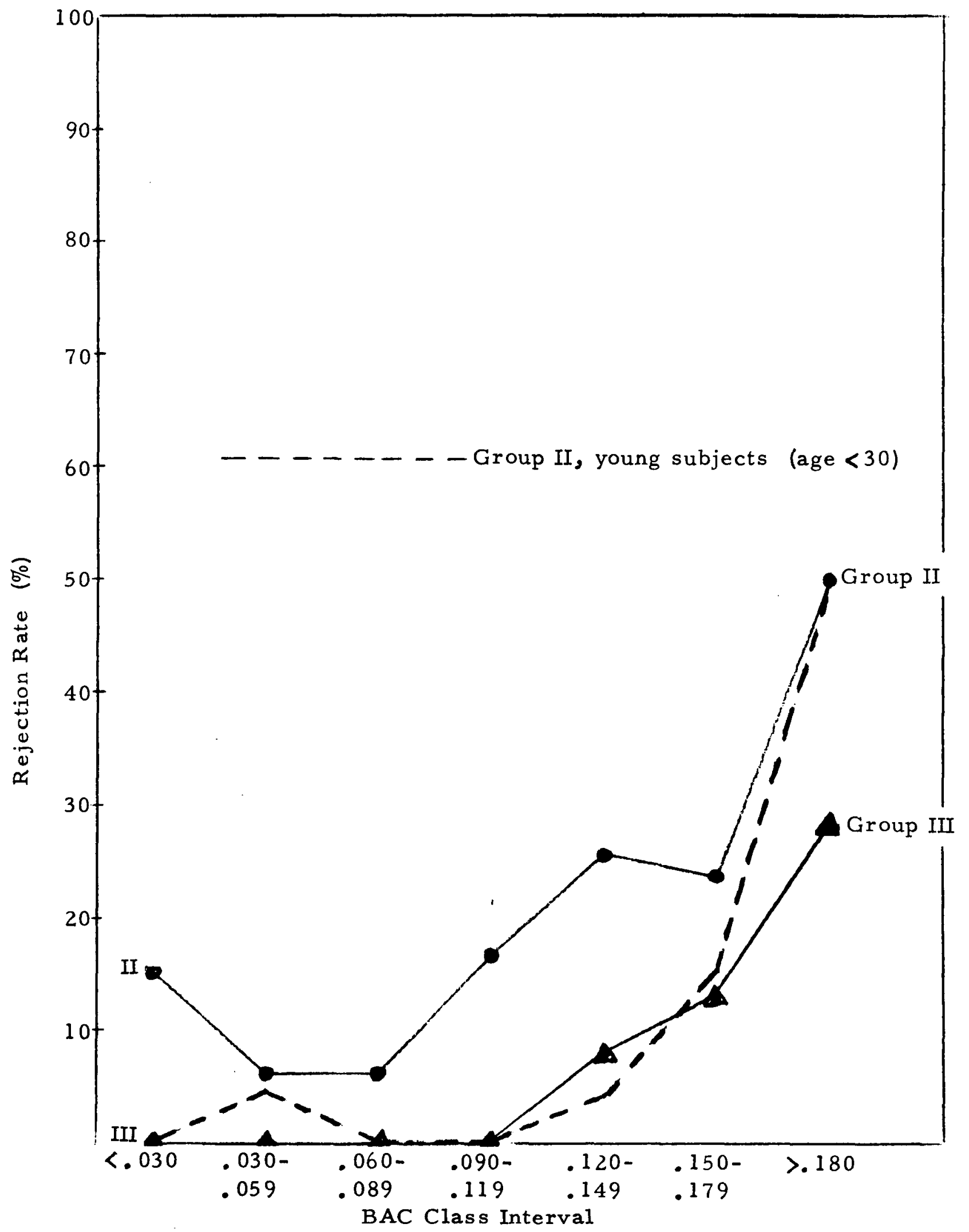

Figure 14. Reaction Analyzer Performance - 2/3 Sirategy 


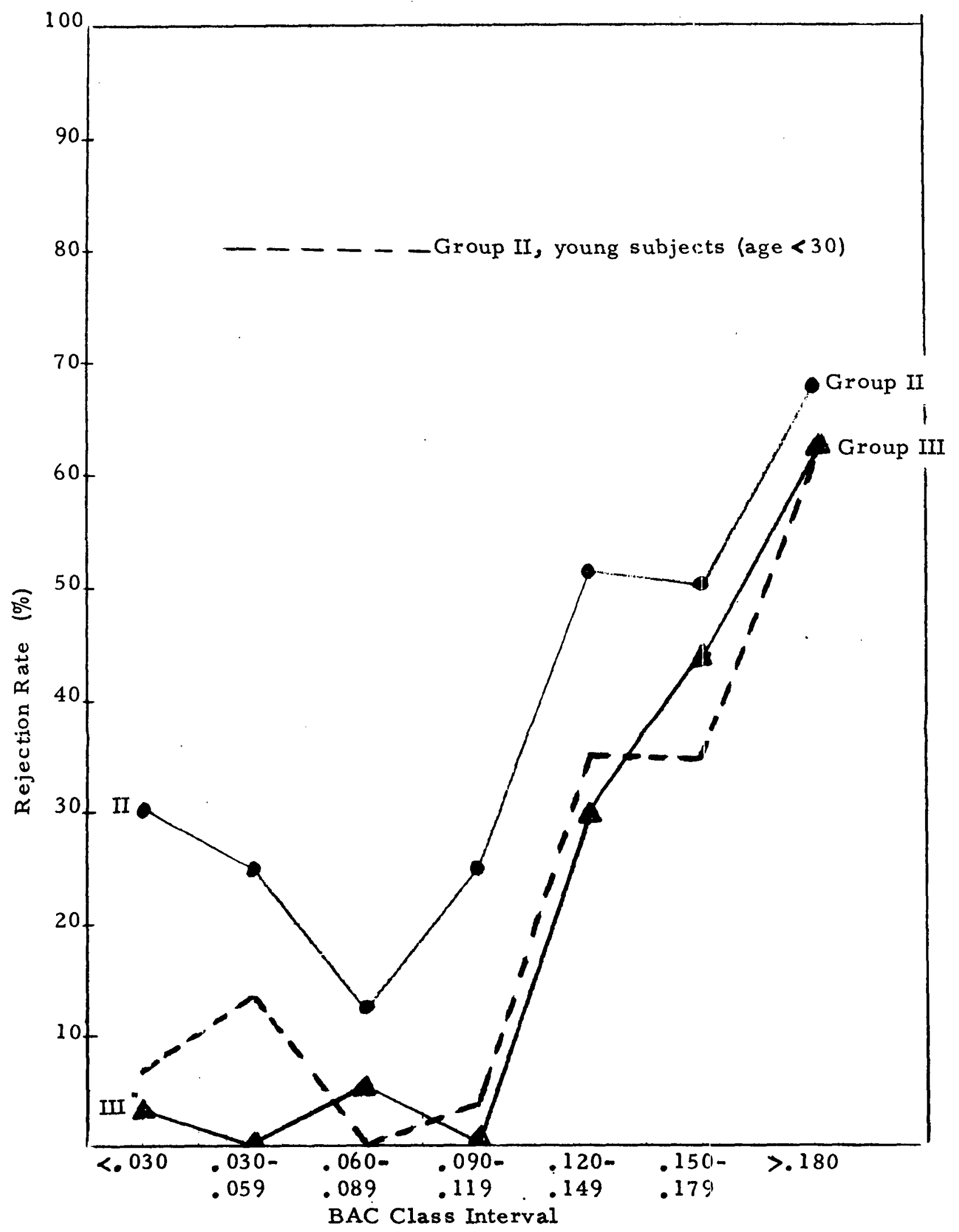

Figure 15. Reaction Analyzer Performance - 3/3 Strategy 


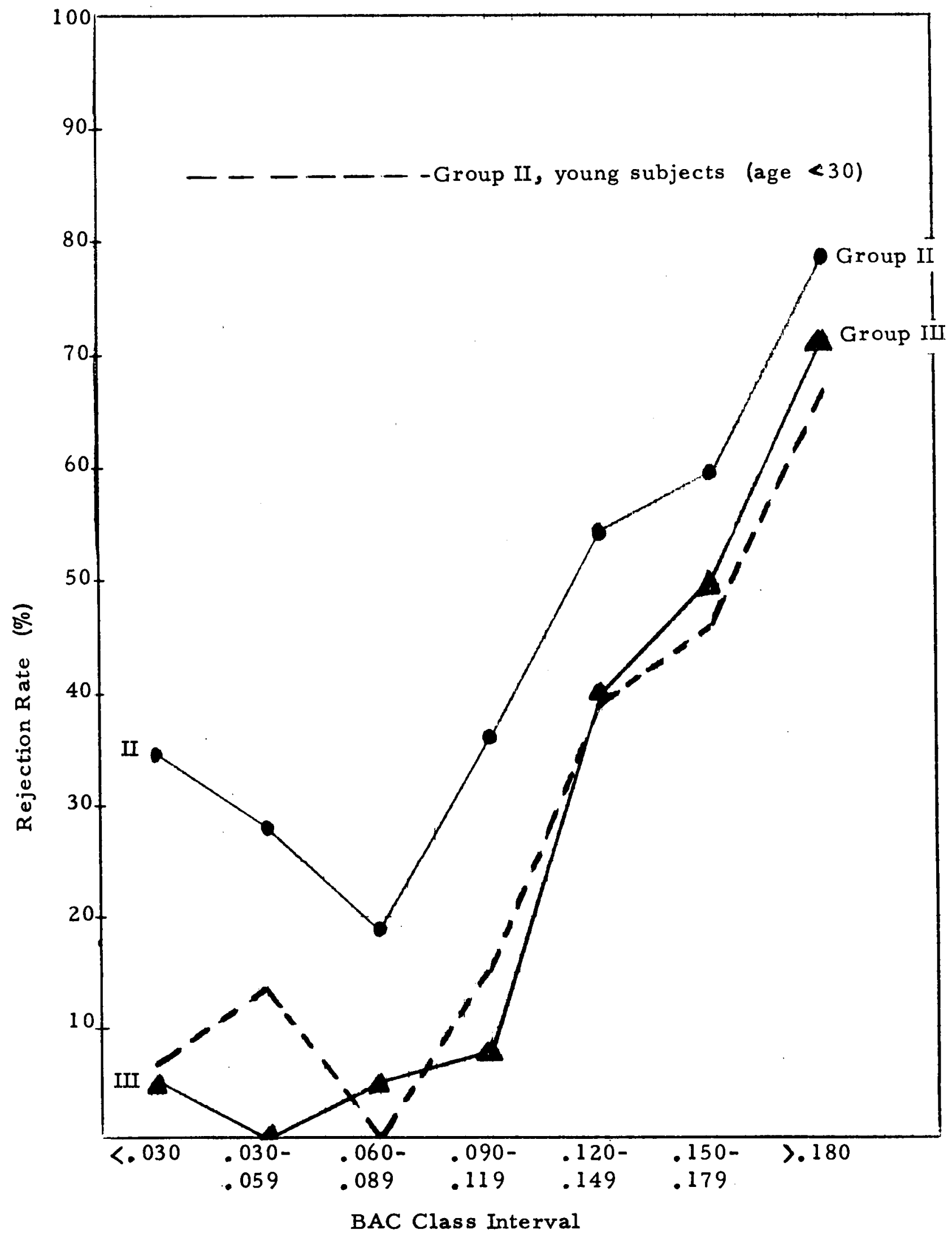

Figure 16. Reaction Analyzer Performance - 5/5 Strategy 


\begin{tabular}{|c|c|c|c|c|c|}
\hline & \multicolumn{2}{|c|}{ BAC vs. SEX } & \multicolumn{2}{|c|}{ BAC vs. AGE } \\
\hline & & $\mathrm{BAC}$ & SEX & $\mathrm{BAC}$ & $\mathrm{AGE}$ \\
\hline $\begin{array}{l}\text { Group } \\
\text { II }\end{array}$ & $\begin{array}{l}F \\
\text { Significance }\end{array}$ & $\begin{array}{c}9.24 \\
.01>p>.001\end{array}$ & $\begin{array}{c}1.60 \\
p=.20\end{array}$ & $\begin{array}{c}2.08 \\
.20=p=.10\end{array}$ & $\begin{array}{c}11.25 \\
.05>p>.01\end{array}$ \\
\hline $\begin{array}{l}\text { Group } \\
\text { III }\end{array}$ & $\begin{array}{l}F \\
\text { Significance }\end{array}$ & $\begin{array}{c}6.75 \\
.05=p=.01\end{array}$ & $\begin{array}{l}0.47 \\
\text { N.S. }\end{array}$ & 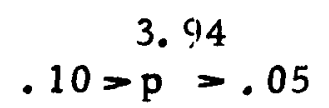 & $\begin{array}{l}0.02 \\
\text { N.S. }\end{array}$ \\
\hline
\end{tabular}

Control session data show relatively high rejection rates during test cycles 1 and 2 , indicating that Subject anxiety also influences Reaction Analyzer performance. No effects of fatigue, however, are evidenced. It will also be noted that maximum control session rejection rate was achieved on cycle $5(8.6 \%)$; however, four of the six failed trials occurring on that cycle were contributed by a single Subject (No. 138) who subsequently stated he had momentarily forgotten how to properly regulate the control knob. As was the case for Quickey, Reaction Analyzer rejection rates produced during control sessions were appreciably higher than those evidenced during drinking sessions prior to ingestion of alcohol.

Figure 17 compares the proportions of trials failed during control and drinking sessions at each test cycle. The mean BAC of each drinking session cycle is also noted. Analysis of variance on these data indicate no significant effects even though the interaction seen in the curves of Figure 17 is only moderate. 


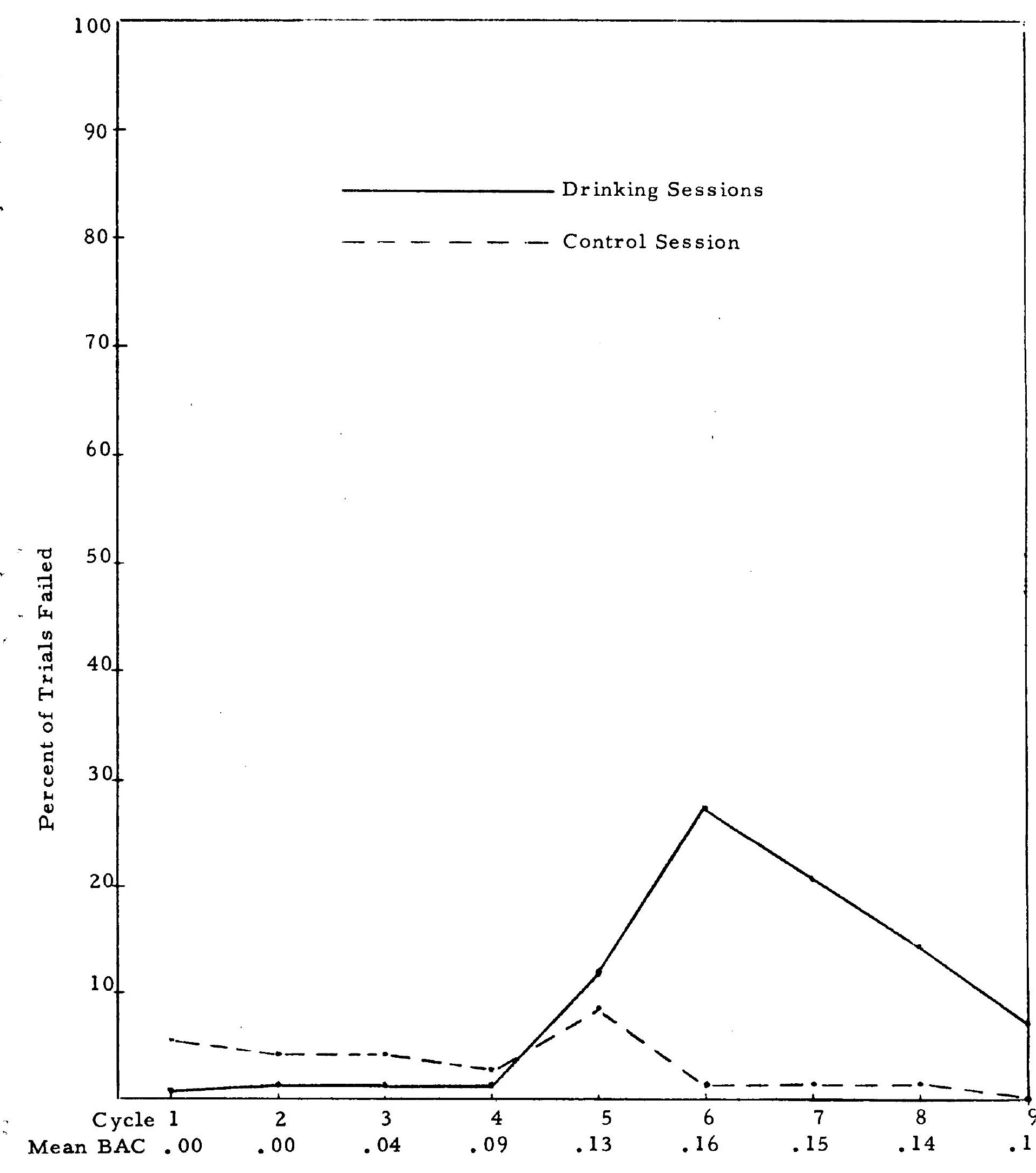

Figure 17. Reaction Analyzer - Comparison of Control and Drinking Sessions - 1/1 Strategy - Group III 
Table XX

Reaction Analyzer Performance, Group II

STRATEGY

\begin{tabular}{|c|c|c|c|c|c|c|c|c|c|c|c|}
\hline \multirow{2}{*}{$\begin{array}{l}\text { SUBJECT } \\
\text { CATEGORY }\end{array}$} & \multirow{2}{*}{$\begin{array}{l}\text { BAC CLASS } \\
\text { INTER VAL }\end{array}$} & \multicolumn{2}{|c|}{$1 / 1$} & \multicolumn{2}{|c|}{$5 / 5$} & \multicolumn{2}{|c|}{$4 / 5$} & \multicolumn{2}{|c|}{$3 / 3$} & \multicolumn{2}{|c|}{$2 / 3$} \\
\hline & & $\mathrm{N}$ & $\%$ & $\mathrm{~N}$ & $\%$ & $\mathrm{~N}$ & $\%$ & $\mathrm{~N}$ & $\%$ & $\mathrm{~N}$ & $\%$ \\
\hline \multirow{7}{*}{$\begin{array}{l}\text { ALL } \\
\text { SUBJECTS }\end{array}$} & $<0.030 \%$ & $32 / 230$ & 13.9 & $16 / 46$ & 34.8 & $10 / 46$ & 21.7 & $14 / 46$ & 30.4 & $7 / 46$ & 15.2 \\
\hline & $0.030-0.059 \%$ & $14 / 160$ & 8.8 & $9 / 32$ & 28.1 & $3 / 32$ & 9.4 & $8 / 32$ & 25,0 & $2 / 32$ & 6.3 \\
\hline & $0.060-0.089 \%$ & $6 / 80$ & 7.5 & $3 / 16$ & 18.8 & $1 / 16$ & 6.3 & $2 / 16$ & 12.5 & $1 / 16$ & 6.3 \\
\hline & $0.090-0.119 \%$ & $28 / 180$ & 15.6 & $13 / 36$ & 36.1 & $7 / 36$ & 19.4 & $9 / 36$ & 25.0 & $6 / 36$ & 16.7 \\
\hline & $0.120-0.149 \%$ & $46 / 175$ & 26.3 & $19 / 35$ & 54.3 & $13 / 35$ & 37.1 & $18 / 35$ & 51.4 & $9 / 35$ & 25.7 \\
\hline & $0.150-0.179 \%$ & $62 / 210$ & 29.5 & $25 / 42$ & 59.5 & $18 / 42$ & 42.9 & $21 / 42$ & 50.0 & $10 / 42$ & 23.8 \\
\hline & $=0.180 \%$ & $64 / 140$ & 45.7 & $22 / 28$ & 78.6 & $18 / 28$ & 64.3 & $19 / 28$ & 67.9 & $14 / 28$ & 50.0 \\
\hline \multirow{7}{*}{ MALES } & $<0.030 \%$ & $7 / 125$ & 5.6 & $5 / 25$ & 20.0 & $2 / 25$ & 8.0 & $4 / 25$ & 16.0 & $0 / 25$ & 0.0 \\
\hline & $0.030-0.059 \%$ & $8 / 80$ & 10.0 & $6 / 16$ & 37.5 & $2 / 16$ & 12.5 & 5.116 & 31.3 & $1 / 16$ & 6.3 \\
\hline & $0.060-0.089 \%$ & $1 / 30$ & 3.3 & $1 / 6$ & 16.7 & $0 / 6$ & 0.0 & $1 / 6$ & 16.7 & $0 / 6$ & 0.0 \\
\hline & $0.090-0.119 \%$ & $12 / 85$ & 14.1 & $6 / 17$ & 35.3 & $3 / 17$ & 17.6 & $3 / 17$ & 17.6 & $2 / 17$ & 11.8 \\
\hline & $0.120-0.149 \%$ & $16 / 90$ & 17.8 & $8 / 18$ & 44.4 & $5 / 18$ & 27.8 & $8 / 18$ & 44.4 & $2 / 18$ & 11.1 \\
\hline & $0.150-0.179 \%$ & $32 / 115$ & 27.8 & $13 / 23$ & 56.5 & $10 / 23$ & 43.5 & $11 / 23$ & 47.8 & $5 / 23$ & 21.7 \\
\hline & $=0.180 \%$ & $34 / 90$ & 37.8 & $12 / 18$ & 66.7 & $12 / 18$ & 66.7 & $11 / 18$ & 61.1 & $9 / 18$ & 50.0 \\
\hline \multirow{7}{*}{ FEMALES } & $<0.030 \%$ & $25 / 105$ & 23.8 & $11 / 21$ & 52.4 & $8 / 21$ & 38.1 & $10 / 21$ & 47.6 & $7 / 21$ & 33.3 \\
\hline & $0.030-0.059 \%$ & $6 / 80$ & 7.5 & $3 / 16$ & 18.8 & $1 / 16$ & 6.3 & $3 / 16$ & 18.8 & $1 / 16$ & 6.3 \\
\hline & $0.060-0.089 \%$ & $5 / 50$ & 10,0 & $2 / 10$ & 20.0 & $1 / 10$ & 10.0 & $1 / 10$ & 10.0 & $1 / 10$ & 10.0 \\
\hline & $0.090-0.119 \%$ & $16 / 95$ & 16.8 & $7 / 19$ & 36.8 & $4 / 19$ & 21.1 & $6 / 19$ & 31.6 & $4 / 19$ & 21.1 \\
\hline & $0.120-0.149 \%$ & $30 / 85$ & 35.3 & $11 / 17$ & 64.7 & $8 / 17$ & 47. 1 & $10 / 17$ & 58.8 & $7 / 17$ & 41.2 \\
\hline & $0.150-0.179 \%$ & $30 / 95$ & 31.6 & $12 / 19$ & 63.2 & $8 / 19$ & 42.1 & $10 / 19$ & 52.6 & $5 / 19$ & 26.3 \\
\hline & $=0.180 \%$ & $30 / 50$ & 60.0 & $10 / 10$ & 100.0 & $6 / 10$ & 60.0 & $8 / 10$ & 80.0 & $5 / 10$ & 50.0 \\
\hline \multirow{7}{*}{$\begin{array}{l}\text { AGE } \\
=30\end{array}$} & $<0.030 \%$ & $3 / 150$ & 2.0 & $2 / 30$ & 6.7 & $1 / 30$ & 3.3 & $2 / 30$ & 6.7 & $0 / 30$ & 0.0 \\
\hline & $0.030-0.059 \%$ & $4 / 110$ & 3.6 & $3 / 22$ & 13.6 & $1 / 22$ & 4.5 & $3 / 22$ & 13.6 & $1 / 22$ & 4.5 \\
\hline & $0.060-0.089 \%$ & $0 / 55$ & 0.0 & $0 / 11$ & 0.0 & $0 / 11$ & 0.0 & $0 / 11$ & 0.0 & $0 / 11$ & 0.0 \\
\hline & $0.090-0.119 \%$ & $4 / 130$ & 3.1 & $4 / 26$ & 15.4 & $0 / 26$ & 0.0 & $1 / 26$ & 3.8 & $0 / 26$ & 0.0 \\
\hline & $0.120-0.149 \%$ & $15 / 115$ & 13.0 & $9 / 23$ & 39.1 & $5 / 23$ & 21.7 & $8 / 23$ & 34.8 & $1 / 23$ & 4.3 \\
\hline & $0.150-0.179 \%$ & $27 / 130$ & 20.8 & $12 / 26$ & 46.2 & $7 / 26$ & 26.9 & $9 / 26$ & 34.6 & $4 / 26$ & 15.4 \\
\hline & $\geq 0.180 \%$ & $34 / 90$ & 37.8 & $12 / 18$ & 66.7 & $12 / 18$ & 66.7 & $11 / 18$ & 61.1 & $9 / 18$ & 50.0 \\
\hline \multirow{7}{*}{$\begin{array}{l}\mathrm{AGE} \\
\geq 30\end{array}$} & $<0.030 \%$ & $29 / 80$ & 36.3 & $14 / 16$ & 87.5 & $9 / 16$ & 56.3 & $12 / 16$ & 75.0 & $7 / 16$ & 43.8 \\
\hline & $0.030-0.059 \%$ & $10 / 50$ & 20.0 & $6 / 10$ & 60.0 & $2 / 10$ & 20.0 & $5 / 10$ & 50.0 & $1 / 10$ & 10.0 \\
\hline & $0.060-0.089 \%$ & $6 / 25$ & 24.0 & $3 / 5$ & 60.0 & $1 / 5$ & 20.0 & $2 / 5$ & 40.0 & $1 / 5$ & 20.0 \\
\hline & $0.090-0.119 \%$ & $24 / 50$ & 48.0 & $9 / 10$ & 90.0 & $7 / 10$ & 70.0 & $8 / 10$ & 80.0 & $6 / 10$ & 60.0 \\
\hline & $0.120-0.149 \%$ & $31 / 60$ & 51.7 & $10 / 12$ & 83.3 & $8 / 12$ & 66.7 & $10 / 12$ & 83.3 & $8 / 12$ & 66.7 \\
\hline & $0.150-0.179 \%$ & $35 / 80$ & 43.8 & $13 / 16$ & 81.3 & $11 / 16$ & 68.8 & $12 / 1.6$ & 75.0 & $6 / 16$ & 37.5 \\
\hline & $=0.180 \%$ & $30 / 50$ & 60.0 & $10 / 10$ & 100.0 & $6 / 10$ & 60.0 & $8 / 10$ & 80.0 & $5 / 10$ & 50.0 \\
\hline
\end{tabular}


STRATEGY

\begin{tabular}{|c|c|c|c|c|c|c|c|c|c|c|c|}
\hline \multirow{2}{*}{$\begin{array}{l}\text { SUBJECT } \\
\text { CATEGORY }\end{array}$} & \multirow{2}{*}{$\begin{array}{l}\text { BAC CLASS } \\
\text { INTER VAL }\end{array}$} & \multirow{2}{*}{\multicolumn{2}{|c|}{$1 / 1$}} & \multicolumn{2}{|c|}{$5 / 5$} & \multicolumn{2}{|c|}{$4 / 5$} & \multicolumn{2}{|c|}{$3 / 3$} & \multicolumn{2}{|c|}{$2 / 3$} \\
\hline & & & & $\mathbf{N}$ & $\%$ & $\mathrm{~N}$ & $\%$ & $\mathbf{N}$ & $\%$ & $\mathbf{N}$ & $\%$ \\
\hline \multirow{7}{*}{$\begin{array}{l}\text { ALL } \\
\text { SUBJECTS }\end{array}$} & $=0.030 \%$ & $3 / 295$ & 1.0 & $3 / 59$ & 5,1 & $0 / 59$ & 0.0 & $2 / 59$ & 3,4 & $0 / 59$ & 0,0 \\
\hline & $0: 030-0.059 \%$ & $0 / 120$ & 0.0 & $0 / 24$ & 0,0 & $0 / 24$ & 0.0 & $0 / 24$ & 0.0 & $0 / 24$ & 0.0 \\
\hline & $0.060-0.089 \%$ & $2 / 95$ & 2.1 & $1 / 19$ & 5.3 & $1 / 19$ & 5.3 & $1 / 19$ & 5.3 & $0 / 19$ & 0,0 \\
\hline & $0.090-0.119 \%$ & $3 / 180$ & 1.7 & $3 / 36$ & 8.3 & $0 / 36$ & 0.0 & $3 / 36$ & 0.0 & $0 / 36$ & 0.0 \\
\hline & $0.120-0.149 \%$ & $34 / 245$ & 13.9 & $20 / 49$ & 40.8 & $11 / 49$ & 22.4 & $15 / 49$ & 30.6 & $4 / 49$ & 8.2 \\
\hline & $0.150-0.179 \%$ & $48 / 260$ & 18.5 & $26 / 52$ & 50.0 & $15 / 52$ & 28.8 & $23 / 52$ & 44.2 & $7 / 52$ & 13.5 \\
\hline & $\geq 0.180 \%$ & $31 / 105$ & 29.5 & $15 / 21$ & 71.4 & 8.21 & 38.1 & $13 / 21$ & 61.9 & $6 / 21$ & 28.6 \\
\hline \multirow{7}{*}{ MALES } & $<0.030 \%$ & $0 / 180$ & 0.0 & $0 / 36$ & 0.0 & $0 / 36$ & 0.0 & $0 / 36$ & 0.0 & $0 / 36$ & 0.0 \\
\hline & $0.030-0.059 \%$ & $0 / 85$ & 0.0 & $0 / 17$ & 0.0 & $0 / 17$ & 0.0 & $0 / 17$ & 0.0 & $0 / 17$ & 0.0 \\
\hline & $0.060-0.089 \%$ & $0 / 60$ & 0.0 & $0 / 12$ & 0.0 & $0 / 12$ & 0.0 & $0 / 12$ & 0.0 & $0 / 12$ & 0.0 \\
\hline & $0.090-0.119 \%$ & $3 / 140$ & 2.1 & $3 / 28$ & 10.7 & $0 / 28$ & 0.0 & $3 / 28$ & 10.7 & $0 / 28$ & 0.0 \\
\hline & $0.120-0.149 \%$ & $26 / 170$ & 15.3 & $14 / 34$ & 41.2 & $9 / 34$ & 26.5 & $10 / 34$ & 29.4 & $3 / 34$ & 8.8 \\
\hline & $0.150-0.179 \%$ & $30 / 150$ & 20.0 & $16 / 30$ & 53.3 & $9 / 30$ & 30.0 & $15 / 30$ & 50.0 & $5 / 30$ & 16.7 \\
\hline & $=0.180 \%$ & $14 / 55$ & 25.5 & $h / 11$ & 63.6 & $3 / 11$ & 27.3 & $6 / 11$ & 54.5 & $3 / 11$ & 27.3 \\
\hline \multirow{7}{*}{ FEMALES } & $<0.030 \%$ & $3 / 115$ & 2.6 & $3 / 23$ & 13.0 & $0 / 23$ & 0.0 & $2 / 23$ & 8.7 & $0 / 23$ & 0.0 \\
\hline & $0.030-0.059 \%$ & $0 / 35$ & 0.0 & $0 / 7$ & 0.0 & $0 / 7$ & 0.0 & $0 / 7$ & 0.0 & $0 / 7$ & 0.0 \\
\hline & $0.060-0.089 \%$ & $2 / 35$ & 5.7 & $1 / 7$ & 14.3 & $1 / 7$ & 14.3 & $1 / 7$ & 14.3 & $0 / 7$ & 0.0 \\
\hline & $0.090-0.119 \%$ & $0 / 40$ & 0.0 & $0 / 8$ & 0.0 & $0 / 8$ & 0.0 & $0 / 8$ & 0.0 & $0 / 8$ & 0.0 \\
\hline & $0.120-0.149 \%$ & $8 / 75$ & 10.7 & $6 / 15$ & 40.0 & $2 / 15$ & 13.3 & $5 / 15$ & 33.3 & $1 / 15$ & 6.7 \\
\hline & $0.150-0.179 \%$ & $18 / 110$ & 16.4 & $10 / 22$ & 45.5 & $6 / 22$ & 27.3 & $8 / 22$ & 36.4 & $2 / 22$ & 9.1 \\
\hline & $\geq 0.180 \%$ & $17 / 50$ & 34.0 & $8 / 10$ & 80.0 & $5 / 10$ & 50.0 & $7 / 10$ & 70.0 & $3 / 10$ & 30.0 \\
\hline \multirow{7}{*}{$\begin{array}{l}\text { AGE } \\
=30\end{array}$} & $=0.030 \%$ & $1 / 125$ & 0.8 & $1 / 25$ & 4.0 & $0 / 25$ & 0.0 & $0 / 25$ & e.e & $0 / 25$ & 0.0 \\
\hline & $0.030-0.059 \%$ & $0 / 70$ & 0.0 & $0 / 14$ & 0.0 & $0 / 14$ & 0.0 & $0 / 14$ & 0.2 & $0 / 14$ & 0.0 \\
\hline & $0.060-0.089 \%$ & $0 / 35$ & 0.0 & $0 / 7$ & 0.0 & $0 / 7$ & 0.0 & $0 / 7$ & 0.0 & $0 / 7$ & 0.0 \\
\hline & $0.090-0.119 \%$ & $0 / 65$ & 0.0 & $0 / 13$ & 0.0 & $0 / 13$ & 0.0 & $0 / 13$ & 0.0 & $0 / 13$ & 0.0 \\
\hline & $0.120-0.149 \%$ & $24 / 145$ & 16.6 & $12 / 29$ & 41.4 & $9 / 29$ & 31.0 & $9 / 29$ & 31.0 & $3 / 29$ & 10.3 \\
\hline & $0.150-0.179 \%$ & $25 / 115$ & 21.7 & $12 / 23$ & 52.2 & $8 / 23$ & 34.8 & $11 / 23$ & 47,8 & $4 / 23$ & 17.4 \\
\hline & $\geq 0.180 \%$ & $12 / 45$ & 26.7 & $7 / 9$ & 77.8 & $2 / 9$ & 22.2 & $5 / 9$ & 55.6 & $2 / 9$ & 22.2 \\
\hline \multirow{7}{*}{$\begin{array}{l}A G E \\
=30\end{array}$} & $<0.030 \%$ & $2 / 170$ & 1.2 & $2 / 34$ & 5.9 & $0 / 34$ & 0.0 & $2 / 34$ & 5.9 & $0 / 34$ & 0.0 \\
\hline & $0.030-0.059 \%$ & $0 / 50$ & 0.0 & $0 / 10$ & 0.0 & $0 / 10$ & 0.0 & $0 / 10$ & 0.0 & $0 / 10$ & 0.0 \\
\hline & $0.060-0.089 \%$ & $2 / 60$ & 3.3 & $1 / 12$ & 8.3 & $1 / 12$ & 8.3 & $1 / 12$ & 8.3 & $0 / 12$ & 0.0 \\
\hline & $0.090-0.119 \%$ & $3 / 115$ & 2.6 & $3 / 23$ & 13.0 & $0 / 23$ & 0.0 & $3 / 23$ & 13.0 & $0 / 23$ & 0.0 \\
\hline & $0.120-0.149 \%$ & $10 / 100$ & 10.0 & $8 / 20$ & 40.0 & $2 / 20$ & 10.0 & $6 / 20$ & 30.0 & $1 / 20$ & 5.0 \\
\hline & $0.150-0.179 \%$ & $23 / 145$ & 15.9 & $14 / 29$ & 48.3 & $7 / 29$ & 24.1 & $12 / 29$ & 41.4 & $3 / 29$ & 10.3 \\
\hline & $=0.180 \%$ & $19 / 60$ & 31.7 & $8 / 12$ & 66.7 & $6 / 12$ & 50.0 & $8 / 12$ & 66.7 & $4 / 12$ & 33.3 \\
\hline
\end{tabular}


Table XXII

Reaction Analyzer Control Session Performance „Group III

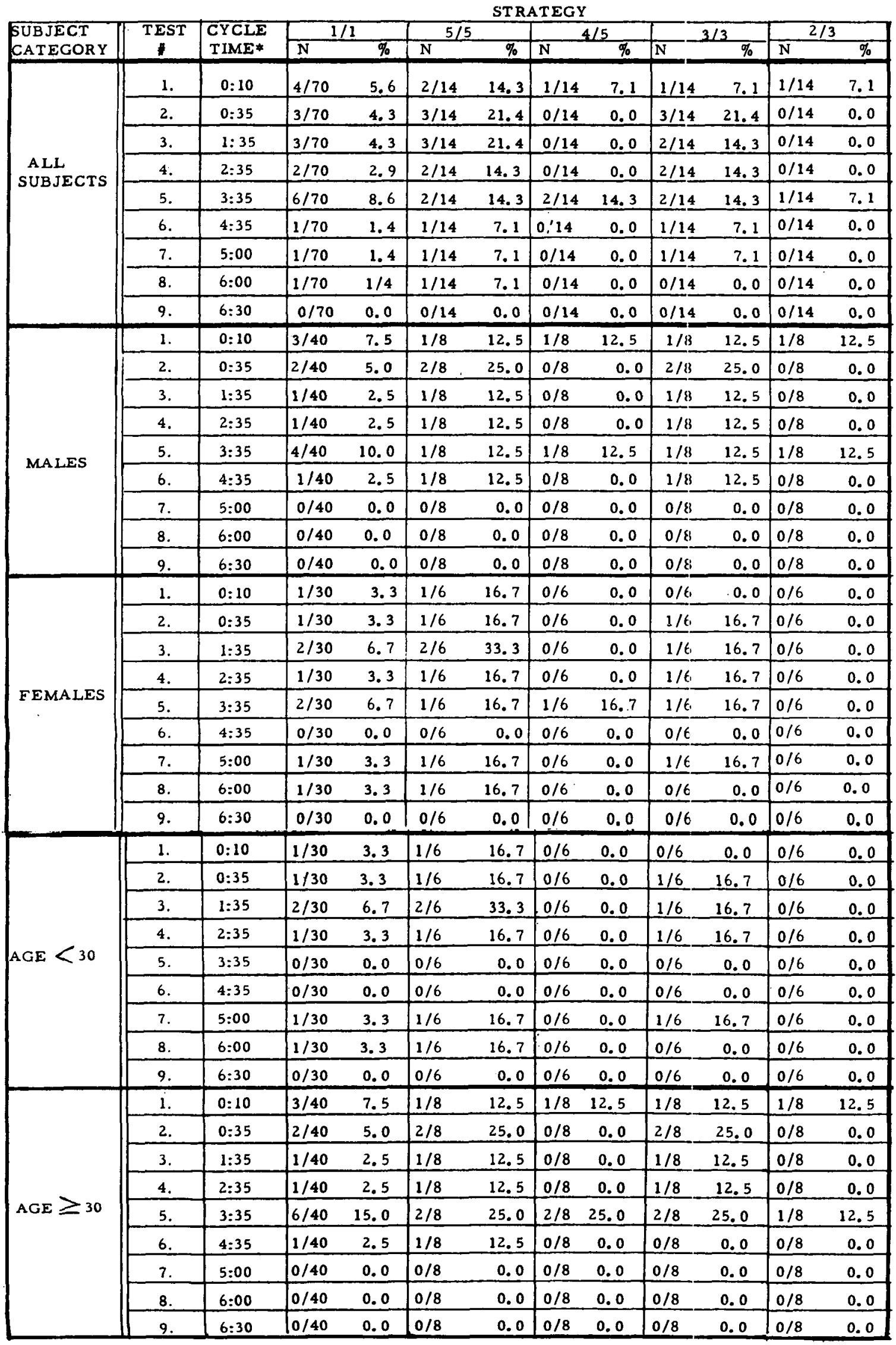


VI. NARTRON

The ASIS developed by Nartron, Inc., consists of an "adding machine"type keyboard with sixteen numeral buttons (0 through 15) and four indicator lights designated "8", "4", "2", and "1." The Subject initiates the trial by closing an on/off switch and entering a code number in the keyboardw. This immediately causes an unpredictable subset (ranging from none to all) of the four indicators to flash. The Subject must mentally compute the total of the illuminated values and depress the corresponding pushbutton.

To pass the trial, the Subject must depress the correct pushbutton within a pre-set response time tolerance. This tolerance is selected for each Subject on an individual basis from among the following possibilities: 1.15 seconds, 1.25 seconds, 1.50 seconds, 1.75 seconds, 2.00 seconds, and 2.25 seconds. "PASS" is displayed via illumination of an indicator labelled "FASTEN SEAT BELTS." "FAIL" is displayed in a manner which indicates its cause: if an incorrect pushbutton has been depressed, a yellow indicator illuminates; if a too slow (but correct) response is the cause, neither indicator lights.

\section{A. Training Procedures}

The Nartron device was involved in the program only for the first group of Subjects, each of whom completed up to 8 blocks of 25 trials during each training session. The first four blocks were designed to determine the Subject's response time training criterion for that session. Block \#1 commenced at a response time of 2.25 seconds; as soon as a trial was passed, the Subject advanced to the 2.00 second setting, and so on through settings of $1.75,1.50$, 1.25, and 1.15 seconds. When a trial was failed, the Subject reverted to the previous setting. Block \#2 began at the 1.15 second setting, with the Subject reverting to 1.25 seconds when a trial was failed. Subsequent passes or failures dictated that the Subject proceed to the next lower or higher time setting, respectively. Block \#3 was identical to Block \#1, and Block \#4 to Block \#2. "Pass" or "Fail" was recorded for each trial. Once the first four blocks had been completed, a member of the project staff arranged each block's passes in a matrix describing the order in which they occurred and the appropriate response time setting, as exemplified by the following sketch:

* The purpose of this code number is as an anti-theft feature of the ASIS. Accuracy of code number entry did not enter into determining whether a Subject passed or failed a given trial. 


Response
Time \begin{tabular}{|l|l|l|l|l|l|l|l|l|l|l|l|l|l|l|l}
1.15 & & & & & & & & & & & $\mathrm{x}$ & & \\
\hline 1.25 & & & & & & $\mathrm{x}$ & & & $\mathrm{x}$ & $\mathrm{x}$ & & & \\
\hline 1.50 & & & & & $\mathrm{x}$ & & & $\mathrm{x}$ & & & & $\mathrm{x}$ & \\
\hline 1.75 & & & & $\mathrm{x}$ & & & $\mathrm{x}$ & & & & & & \\
\hline 2.00 & & $\mathrm{x}$ & $\mathrm{x}$ & & & & & & & & & & \\
\hline 2.25 & $\mathrm{x}$ & & & & & & & & & & & & \\
\hline
\end{tabular}

The median response time for all passes of the block was next computed (in the above sketch, this would be 1.50 seconds). The average of the medians of the four blocks (rounded up to the nearest specific setting among the set of six) defined the training criterion for the remainder of that session. The Subject then completed up to an additional four blocks, with all trials taking place at the criterion setting. Subjects received $\$ 5.00$ reward for passing at least 23 trials in any block at the assigned criterion setting. Two Subjects achieved reward on all three sessions, three others did so on exactly one of the three sessions, and the remaining five never achieved reward.

Nartron testing criterion was assigned on the basis of the Subject's success during the last four blocks of the third training session. The rules employed to determine criterion may be listed as follows:

- If the Subject passed at least $85 \%$ of the trials, assign the third session's training criterion for testing;

- If the Subject passed at least $75 \%$, but less than $85 \%$, assign the next higher response time setting as the testing criterion;

- If the Subject passed less than $75 \%$ of the trials, assign the 2 nd higher response time setting as the testing criterion.

An exception to this rule was made for Subject No. 107; although passing only $75 \%$ of the third training session's trials at a setting of 1.50 seconds, the Subject had passed $92 \%$ at the same setting during the second session. Accordingly, testing criterion was set at 1.50 seconds in this case. Table XXIII exhibits testing criteria assigned to all Subjects. 
Table XXIII

Nartron Testing Criteria

\begin{tabular}{c||c} 
Subject No. & Criterion (seconds) \\
\hline 101 & 2.25 \\
102 & 1.25 \\
103 & 1.25 \\
104 & 1.50 \\
105 & 1.75 \\
106 & 1.25 \\
107 & 1.50 \\
108 & 1.75 \\
109 & 1.75 \\
110 & 2.00
\end{tabular}




\section{B. Testing Procedures}

Nartron testing employed a block of three trials in each test cycle, with a reward of $\$ 0.50$ for each trial passed. The Escort set the instrument at the appropriate response time criterion for each Subject and recorded each trial as a "Pass" or "Fail."

Midway through the second testing session Subjects began to complain that the device could not be passed whenever two or more of the four stimulus lights flashed. This complaint was voiced continuously during sessions 3 and 4. Subsequent to session 4, a member of the project staff conducted in excess of 200 trials (with the majority of the se at the 2.25 second setting); only 2 multiple stimulus trials were passed, with roughly 150 failed. After discussion with the Contract Technical Manager, a decision was reached to cease Nartron testing. In view of the scarcity of useful data, no analyses of Nartron performance have been conducted. 
The preceding sections have presented tabulations of performance estimates for the candidate ASIS units corresponding to a number of design permutations and alternate strategies. Based upon these data, at least tentative conclusions may be drawn concerning the suitability of these instruments for operational implementations. Suitability is dependent upon the ability of the ASIS to satisfy the objectives and requirements of the particular implementation scheme in question. While a number of schemes are possible, the following two appear most probable at this time:

- Universal application--this scheme, probably initiated through the enactment of a Federal standard, would call for the installation of an ASIS in every new automobile, beginning at some specific model year. This would correspond to the approaches taken to implement seat belts and head restraints in recent years. Ultimately, the Universal application would affect virtually every driver in the nation.

- Limited application--this scheme would require ASIS installation in vehicles operated by a specific segment of the driving population, e.g., individuals who have been convicted of one or more counts of driving while intoxicated or equivalent charge. Such installations might be mandated through the courts, departments of motor vehicles, or other appropriate agencies as a condition for license reinstatement.

Although specific ASIS performance requirements are yet to be determined for these schemes, it is possible to make some qualitiative assessment of their needs. In a Universal application, on the one hand, a device would not necessarily have to produce extremely high rejection rates at elevated $B A C$ in order to merit consideration for use. For example, if a device averaging as low as $25 \%$ performance at and above $0.10 \%$ BAC had been applied universally in Nassau County, New York, during 1959-1970, it might have prevented 55 of the 569 driver fatalities occurring over that period. $k^{2}$ Similar impact on a national scale could result in annual prevention of some

\footnotetext{
The methods and data through which these calculations were derived are presented in the previously-cited report Methodologies for Estimating the Effectiveness of Alcohol Safety Interlock Systems.
} 
5, 000 deaths. However, public acceptance of a Universal ASIS--and thus the feasibility of this scheme--would almost certainly demand negligible rejection rate at zero $B A C$. If a device producing even $0.1 \%$ rejection at this level were presently in use throughout the nation, as many as 250,000 alcohol-free drivers per day might needlessly be prevented from starting their vehicles. *

Under a Limited application, on the other hand, rejection rates at zero $B A C$ on the order of a few percent probably could be tolerated. The fact that the individuals affected by such a scheme would have "brought it on themselves" would be considered by many controlling authorities as justifying the imposition of a certain amount of inconvenience. ** Conversely, since this application would involve only a relatively small segment of the driving population, fairly high rejection rates at elevated BACs would be required if the device were to have a substantial national impact.

In addition to the differing requirements for ASIS performance, the Universal and Limited schemes would be expected to pose differential needs concerning such factors as unit cost, reliability, and maintainability. This experimental program was not intended to address these items, and so it is inappropriate to discuss their impact upon the suitability of the candidate instruments in this report. One additional and important factor.for which data have been obtained concerns training requirements. For Limited applications, it is expected that the State Department of Motor Vehicles, State Police, or some other specific agency will be charged with insuring that each affected driver achieves adequate familiarity with the device prior to installation. Although it is desirable to minimize the resulting additional workload to be borne by such agencies, *** up to a full day's required training for each driver might be tolerated. Under the Universal scheme, however, the necessary training should be sufficiently simple to permit its satisfaction (with little or no supervision) at the automobile dealer's facilities. Anything more than an hour's training likely would prove intolerable for this application.

* The methods and data through which these calculations were derived are presented in the previously-cited report Methodologies for Estimating the Effectiveness of Alcohol Safety Interlock Systems.

***

This was the consensus of some 20 individuals contacted by the authors and representing judicial, law enforcement, motor vehicle administrative, and alcohol countermeasures agencies.

*** Which must, incidentally, be considered part of the cost of a candidate ASIS. 
In view of the considerations listed above, none of the ASIS devices examined in this program appears particularly suited to Universal application. Of the five instruments, only Quickey might conceivably satisfy the minimal requirement for training duration, and the care that apparently must be exercised in establishing the appropriate criterion "window" would preclude entrusting this task to an automobile salesman or the individual himself. In addition, all four devices for which testing was completed admit the possibility of higher rejection rates at zero BAC than would be acceptable for Universal employment.

The possibilities for Limited applications seem much more promising. The Phystester, with 3.0 second response time and strategies of $2 / 2$ or $2 / 3$, and the Reaction Analyzer, under a strategy of $2 / 3,3 / 3$, or $4 / 5$, seem definitely suited to such a scheme, although there is ample room for improved performance in both cases. Quickey, implemented with the adjusted criterion "window" (16th percentile plus $10 \%$ ) and requiring 2 criterion responses within a 2 minute trial, offers very good performance when all Subjects are highly motivated during training as in Group II. When training scores do not uniformly represent the Subjects' ultimate capabilities, however, as in Group III and the special experiment, performance suffers. The Complex Reaction Tester seems least promising of the four devices for which testing was completed. Of the strategies examined, only the $2 / 3$ appears even marginally suited for Limited application, with all other strategies producing unreasonably high rejection rates at zero BAC. However, this instrument should not be eliminated from consideration for future use. Its performance might be improved to the point of operational suitability by slight increase in response time above 0.9 seconds or certain other potentially beneficial design modifications (discussed subsequently in this section).

This report concludes with a listing of recommendations specific to each candidate ASIS unit.

\section{- Complex Reaction Tester}

It is recommended that this unit be submitted to additional laboratory testing to assess the potential benefits of the following two design modifications:

1) Slight increase in response time above 0.9 seconds; Group II testing demonstrated that the present intermediate setting ( 1.8 seconds) represents too large an increase; however, a setting of 1.0 to 1.2 seconds may be more nearly optimum. 
2) Decrease in the time interval between stimulus flashes; the present relatively long duration of this interval (approximately 10 seconds) almost certainly contributes to the anxiety and sense of frustration referred to in Section II. A decrease in the interval might minimize these factors without drastically affecting rejection rate at high BAC (where alcohol impairment presumably is the major cause of failure).

In addition to its possibly beneficial effects upon performance, decrease in the inter-flash interval should also enhance the feasibility of strategies requiring that several trials be attempted. The existing design, with a strategy calling for 3 attempts, would necessitate roughly four minutes of testing each time a driver attempted to start his car. This fact alone might preclude public acceptance of the instrument.

\section{- Phystester}

Although this instrument was found to produce attractive performance with a 3.0 second response time setting, there is some $€$ vidence that a single response time criterion will not be suited to all drivers. As reported in Section III, one member of Group I failed to achieve training criterion on the 3. 6 second device during the course of three training sesisions. It is therefore recommended that an experiment be conducted, using the Group III Phystester training procedures, to determine the proportion of drivers that may be expected to "qualify" on the 3.0 second device. "This will serve to identify the most appropriate technique for implementing the instrument (i.e., individually-set criteria versus a single, generic value).

\section{- Quickey}

The most important issue yet to be resolved for this device concerns its susceptibility to "cheating." Detailed examination of the baseline (training) reaction times produced by the special experiment Subjects* may uncover techniques for detecting purposeful attempts to misrepresent true capabilities. If such techniques can be developed, their effectiveness should be tested against carefully instructed "cheaters" in a la:ger-scale experiment than was

*

The previous experience of these Subjects with Quickey may have enabled them to cheat more effectively than could individuals freshly exposed to the device. Special experiment results thus may overestimate the instrument's susceptibility to this problem. 
conducted in this program. Appropriate remedial actions to be taken in the event that "cheating" is detected must also be identified.

It should be noted that purposeful attempts at "cheating" are not the only threat to Quickey's performance. Group III training results suggest that some Subjects--due to a lack of motivation--did not acquire criteria representative of their ultimate capabilities. It appears possible that such individuals, given additional exposure and the motivation naturally associated with starting one's car, could ultimately acquire the ability to produce scores substantially lower than their assigned criterion "windows." At that point they would become de facto cheaters, although not by prior intent. Thus it is recommended that efforts be directed to developing training procedures providing some control of Subject motivation within the constraints of probable implementation schemes. Group II results demonstrate that, when motivation is uniformly high, appropriate criteria can be readily identified to produce very attractive performance over a wide range of BAC.

\section{- Reaction Analyzer}

The relatively low rejection rate at zero BAC produced by this device-as demonstrated, at least, by the Group III Subjects--suggests that the task might be made slightly more difficult, enhancing performance at high $B A C$, without greatly increasing the proportion of "sober" drivers prevented from starting their vehicles. The developer of the instrument, Mr. Lyle Hill of Raytheon Co., has stated that increased difficulty could be achieved with a relatively minor modification to the existing design. It is suggested that this be implemented and the device submitted to additional laboratory testing.

One issue worthy of further attention concerns the provision of adequate training on this device. Group II experience indicated that some older Subjects had not achieved sufficient facility with the device although they had satisfied the training criterion then employed. A substantial portion of the Subjects selected for any additional testing should be recruited from the over -40 age group to permit identification of training procedures appropriate to such individuals.

\section{- Nartron}

Although insufficient data were obtained to provide an estimate of this instrument's performance, results of the first test session (prior to detection of any problem) indicated some sensitivity to alcohol impairment. It is recommended that this device be resubmitted for laboratory testing, once the problems causing the malfunction have been identified and corrected.

*e.g., the adjusted "window" (16th percentile plus $10 \%$ ) requiring 2 criterion responses within a 2 minute trial. 


\section{APPENDIX A}

\section{OBSERVATIONS OF PHYSIOLOGICAL PHENOMENA RELATED TO ALCOHOL}

The administration of relatively large doses of alcohol and the conduct of re. peated breath tests for monitoring BAC were constant features of this experimental program. As a by-product of this program, data in substantial quantities were obtained concerning the rate of alcohol elimination and the relationship between ethanol dosage and peak BAC. Certain findings were at variance with generally-accepted results. This Appendix discusses these variations and presents the results of analyses conducted to examine potential causes.

In reviewing the results presented herein, it must be remembered that this program was not designed specifically to investigate these phenomena. Thus, no provisions were made for controlling or quantitatively measuring certain factors that, with hindsight, may have affected the phenomena in question. These analyses and their results therefore cannot be considered conclusive, but merely suggestive of possible relationships and the need for further investigation.

\section{Rate of Alcohol Elimination}

There is general agreement in the literature that elimination of blood alcohol is linear with time, subsequent to complete absorption. A number of investigator have concluded that this constant rate of elimination lies in the range from 0.013 to $0.018 \%$ per hour, * although substantial individual variations have been noted. Most sources give $0.015 \%$ per hour as the approximate rate.

Throughout the course of this program, however, it was noted that Subjects' BACs decreased at much higher rates than cited above. Although breath measurement inaccuracies, e.g., a predominance of tidal air in some breath samples, were initially suspected as the cause of this anomaly, the extreme care taken to obtain good, alveolar samples, the remarkable cooperation of the Subjects in this effort, and the usual close agreement among the three breath tests conducted during each test cycle indicate that the observed rates truly reflect the elimination process. Recognizing that previous investigations often dealt

\footnotetext{
* See, for example, Westerfield, W. W. and Schulman, M. P. "Metabolism and Caloric Value of Alcohol" J.Amer Med Assoc, 170, 197-203 (1959); also, Coldwell, B. B. and Smith, H. W. "Alcohol Levels in Body Fluids After Ingestion of Distilled Spirtis" Can J Biochem Physiol, 37, 43-52 (1959).
} 
with peak BACs considerably lower than those achieved in this program, it was hypothesized that elimination rate might depend upon the concentration present. A review of the literature was inconclusive on this point. For example, studies conducted by Coldwell*, Coldwell and Grant**, and Payne, Hill, and King ***;, produced some evidence that the rate increased with BAC; however, other sources $* * * *$ flatly state that such dependence is non-existent. In view of this disagreement, a decision was made to test this hypothesis; with the data obtained from the program.

The test was conducted as follows:

Peak BAC subsequent to complete absorption was estimated from the two GAS Chromatograph (GC) measurements conducted during the test cycle following the last drink. These two measurements and their respective times were averaged to obtain a starting value and time. The GC results and times obtained during the test cycle taking place roughly one hour later were also averaged to obtain a second value and time. The decrease in BAC, divided by the time span, provided an estimate of elimination rate. Since the Breathalyzer was felt to be more susceptible to operator error and the effects of any potential contaminants, no use was made of its results. Thus, no data obtained from the five test sessions during which the GC was inoperative were employed. In a number of other sessions, insufficient testing was conducted after the last drink to permit calculation of elimination rate. A total of sixty-one (61) data points were available. These data are listed in Table A-I.

A linear regression equation was computed between elimination rate and initial BAC. This equation was found to be:

$$
E R=0.224 B A C-0.0122
$$

The corresponding correlation coefficient $(r)$ of 0.57 was significant at the .001 level. Thus, a non-negligible correlation does seem to exist between $B A C$ and elimination rate. This equation and the data points actually obtained are shown in Figure A-1.

* Coldwell, B. B. "A Note on the Estimation and Disappearance of Alcohol in Blood, Breath, and Urine from Obese and Diabetic Patients, "J.Forensic Sci, $10,480-489$, (1965).

***

Coldwell, B. B. and Grant, G. L. "The Disappearance of Alcohol from the

Blood of Diabetics," J. Forensic Sci, 8, 220-230, (1963).

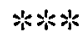

Payne, J. P., Hill, D. W., and King, N. W. , "Observations on the Distribution of Alcohol in Blood, Breath, and Urine, "Brit Med J., 1, 196-202 (1966). ******

Alcohol and the Impaired Driver, American Medical Association, Chicago, 1968; pg. 21 . 
Observed Rates of Elimination, Program Subjects

\begin{tabular}{|c|c|c|c|c|}
\hline Subject & $\begin{array}{r}\text { Initial } \\
\text { BAC } \\
\end{array}$ & $\begin{array}{c}\text { Subsequent } \\
\text { BAC }\end{array}$ & $\begin{array}{c}\text { Time Interval } \\
\text { (Min.) }\end{array}$ & $\begin{array}{c}\text { Rate } \\
\text { (\% per hr.) } \\
\end{array}$ \\
\hline \multirow[t]{3}{*}{101} & .187 & .1505 & 46 & .0477 \\
\hline & .1525 & .1325 & 64 & .0187 \\
\hline & .170 & .1395 & 69 & .0265 \\
\hline \multirow[t]{3}{*}{102} & .1775 & .142 & 81 & .0263 \\
\hline & .185 & .1465 & 71 & .0326 \\
\hline & .157 & .1275 & 62 & .0286 \\
\hline \multirow[t]{3}{*}{103} & .1565 & .131 & 84 & .0182 \\
\hline & .196 & .160 & 62 & .0350 \\
\hline & .202 & .161 & 69 & .0357 \\
\hline \multirow[t]{3}{*}{104} & .1625 & .122 & 81 & .0300 \\
\hline & .170 & .1545 & 49 & .0189 \\
\hline & .179 & .1575 & 68 & .0190 \\
\hline \multirow[t]{2}{*}{105} & .179 & .1555 & 51 & .0276 \\
\hline & .185 & .1535 & 47 & .0417 \\
\hline \multirow[t]{2}{*}{106} & .1855 & .1545 & 83 & .0225 \\
\hline & .1735 & .151 & 53 & .0256 \\
\hline \multirow[t]{2}{*}{107} & $\begin{array}{l}1735 \\
.192\end{array}$ & $\begin{array}{l}.145 \\
.1735\end{array}$ & $\begin{array}{l}88 \\
49\end{array}$ & $\begin{array}{l}.0187 \\
.0226\end{array}$ \\
\hline & .2045 & .1815 & 63 & .0219 \\
\hline \multirow[t]{3}{*}{108} & .183 & .139 & 104 & .0254 \\
\hline & .149 & .1195 & 68 & .0261 \\
\hline & .167 & .1475 & 45 & .0260 \\
\hline \multirow[t]{2}{*}{109} & .1615 & .127 & 83 & .0250 \\
\hline & .193 & .162 & 75 & .0248 \\
\hline \multirow[t]{3}{*}{110} & .164 & .1295 & 82 & .0252 \\
\hline & .145 & .119 & 68 & .0230 \\
\hline & .197 & .1485 & 85 & .0271 \\
\hline \multirow[t]{2}{*}{111} & .188 & .137 & 76 & .0402 \\
\hline & .1805 & .1545 & 59 & .0265 \\
\hline \multirow[t]{3}{*}{112} & .1995 & .1655 & 63 & .0324 \\
\hline & .1855 & .1615 & 51 & .0282 \\
\hline & .201 & .1645 & 57 & .0384 \\
\hline \multirow[t]{3}{*}{114} & .183 & .1385 & 50 & .0536 \\
\hline & .221 & .1795 & 57 & .0437 \\
\hline & .167 & .133 & 45 & .0453 \\
\hline \multirow[t]{2}{*}{115} & .1295 & .1135 & 57 & .0168 \\
\hline & .124 & .1155 & 46 & .0110 \\
\hline
\end{tabular}


Table A-I (Cont'd)

Observed Rates of Elimination, Program Subjects

\begin{tabular}{cllcl} 
Subject & $\begin{array}{c}\text { Initial } \\
\text { BAC }\end{array}$ & $\begin{array}{c}\text { Subsequent } \\
\text { BAC }\end{array}$ & $\begin{array}{c}\text { Time Interval } \\
\text { (Min.) }\end{array}$ & $\begin{array}{c}\text { Rate } \\
\text { (\% per hr.) }\end{array}$ \\
\hline 116 & .1765 & .152 & 80 & .0184 \\
117 & .1225 & .109 & 46 & .0175 \\
& .166 & .155 & 59 & .0112 \\
118 & .1585 & .1245 & 99 & .0206 \\
& .1935 & .1715 & 66 & .0200 \\
119 & .181 & .157 & 95 & .0152 \\
& .189 & .1575 & 68 & .0279 \\
122 & .131 & .1035 & 96 & .0172 \\
& .146 & .107 & 65 & .0361 \\
123 & .184 & .1515 & 63 & .0310 \\
125 & .199 & .163 & 59 & .0367 \\
126 & .1225 & .116 & 48 & .0081 \\
& .183 & .154 & 60 & .0290 \\
128 & .1735 & .1515 & 51 & .0259 \\
130 & .1795 & .1595 & 57 & .0211 \\
134 & .1695 & .146 & .0267 \\
135 & .145 & .130 & .153 & .0158 \\
136 & .133 & .1045 & 57 & .0234 \\
& .130 & .1015 & 73 & .0178 \\
137 & .1885 & .168 & 96 & .0281 \\
138 & .1775 & .1575 & .0204 \\
141 & .1505 & .129 & .0299 \\
& .12045 & .1755 & .0349
\end{tabular}




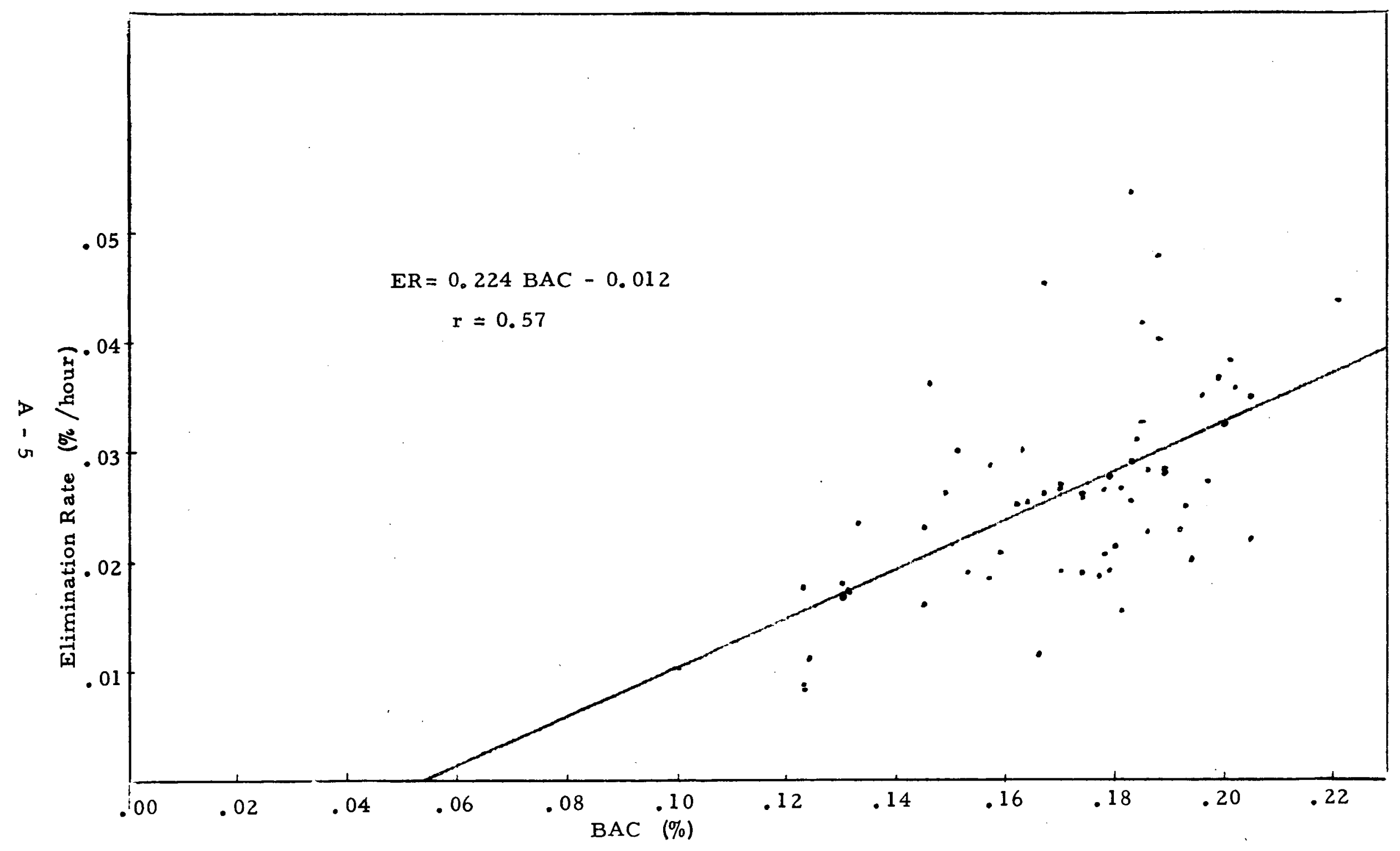

Figure A-1. Elimination Rate Vs. BAC - Dunlap Data 
The equation presented above clearly cannot represent the true elimination rate since it implies that $\mathrm{BAC}$ will never decrease below roughly $0.055 \%$ ! However, this almost certainly occurs because the program's testing paradigm did not offer opportunity to monitor elimination rates at BACs below $0.120 \%$. In order to examine the hypothesis in greater detail, data obtained in the previously-cited study conducted by Coldwell and Grant were accessed. This provided eighteen (18) data points over the BAC range from approximately $0.010 \%$ to $0.080 \%$. These data are presented in Table A-II.

Calculation based upon all seventy-nine (79) data points produced the regression equation:

$$
E R=0.107 \mathrm{BAC}+0.008
$$

with an $r$ value of 0.62 , significant at the .001 level. The correlation thus seemed to be strengthened slightly. The equation and all data points employed are shown in Figure $\mathrm{A}-2$.

It is of interest to note that this second equation implies that, over the BAC range from roughly $0.05 \%$ to $0.10 \%$, elimination rate will be roughly that stated in the literature $(.013 \%-.018 \%$ per hour $)$. This range corresponds to the peak BACs typically attained in many previous studies. The equation further implies that, at very low BACs, elimination rate becomes nearly constant at roughly $0.01 \%$ per hour. This also agrees closely with previous findings. *

Elimination rate is the rate of change in BAC over time (the derivative with respect to time). When rephrased in differential equation format, Eq. A-2 is amenable to solution, providing the following result:

$$
-0.107 \mathrm{~T}
$$

$$
\left.\mathrm{BAC}=\left(\mathrm{BAC}^{\prime}+.075\right) \mathrm{e} \quad-.075 \quad \text { (Eq. } \mathrm{A}-3\right)
$$

where $T$ is the time, in hours, since completion of absorption and BAC' is the concentration at $\mathrm{T}=0$. Figure $\mathrm{A}-3$ depicts this theoretical relationship for initial BAC values of $0.10 \%, 0.15 \%$, and $0.20 \%$.

Although this analysis indicates that a relationship exists between BAC and its rate of elimination, it is possible that additional factors contributed to the high rates observed. In particular, there is some evidence that ingestion of fructose will increase elimination rate; for example, Fletscher, Bernstein

\footnotetext{
"See again Alcohol and the Impaired Driver, pg. 22.
} 
Rates of Elimination - Coldwell and Grant * Subjects

\begin{tabular}{|c|c|c|c|c|}
\hline Subject & $\begin{array}{c}\text { Initial } \\
\text { BAC } \\
\end{array}$ & $\begin{array}{c}\text { Subsequent } \\
\text { BAC } \\
\end{array}$ & $\begin{array}{c}\text { Time Interval } \\
\text { (Min.) }\end{array}$ & $\begin{array}{c}\text { Rate } \\
(\% \text { per } \mathrm{hr} .) \\
\end{array}$ \\
\hline 1 & .043 & .025 & 90 & 0120 \\
\hline 2 & .051 & .027 & 93 & .0154 \\
\hline 3 & .046 & .011 & 75 & .0282 \\
\hline 4 & .042 & .016 & 75 & 0212 \\
\hline 5 & .033 & .004 & 98 & .0178 \\
\hline 6 & .031 & .021 & 65 & .0084 \\
\hline 7 & .042 & .030 & 75 & .0064 \\
\hline 8 & .050 & .027 & 75 & 0184 \\
\hline 9 & .080 & .047 & 80 & 0247 \\
\hline 10 & .029 & .008 & 75 & .0167 \\
\hline 11 & .019 & .002 & 95 & .0107 \\
\hline 12 & .031 & .019 & 65 & .0111 \\
\hline 13 & .009 & .000 & 75 & .0072 \\
\hline 14 & .049 & .040 & 65 & 0090 \\
\hline 15 & .035 & .027 & 87 & 0053 \\
\hline 16 & .081 & .053 & 80 & .0206 \\
\hline 17 & .067 & .040 & 85 & .0187 \\
\hline 18 & .032 & .017 & 60 & .0148 \\
\hline
\end{tabular}

*Coldwell, B. B. and Grant, G. L., Op. Cit (1963). 


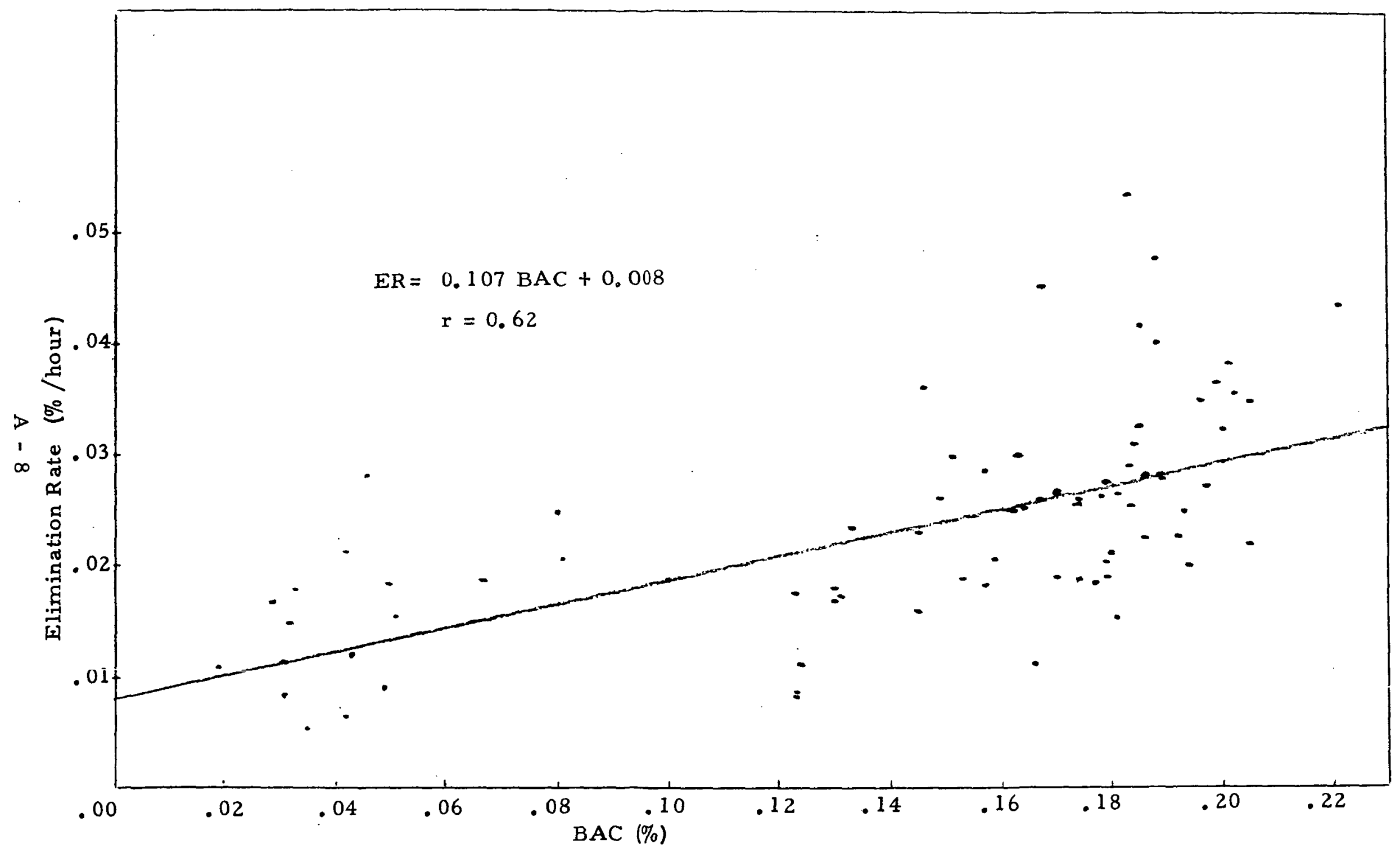

Figure A-2. Elimination Rate Vs. BAC - Dunlap Data Augmented by Coldwell and Grant (1963) Data. 


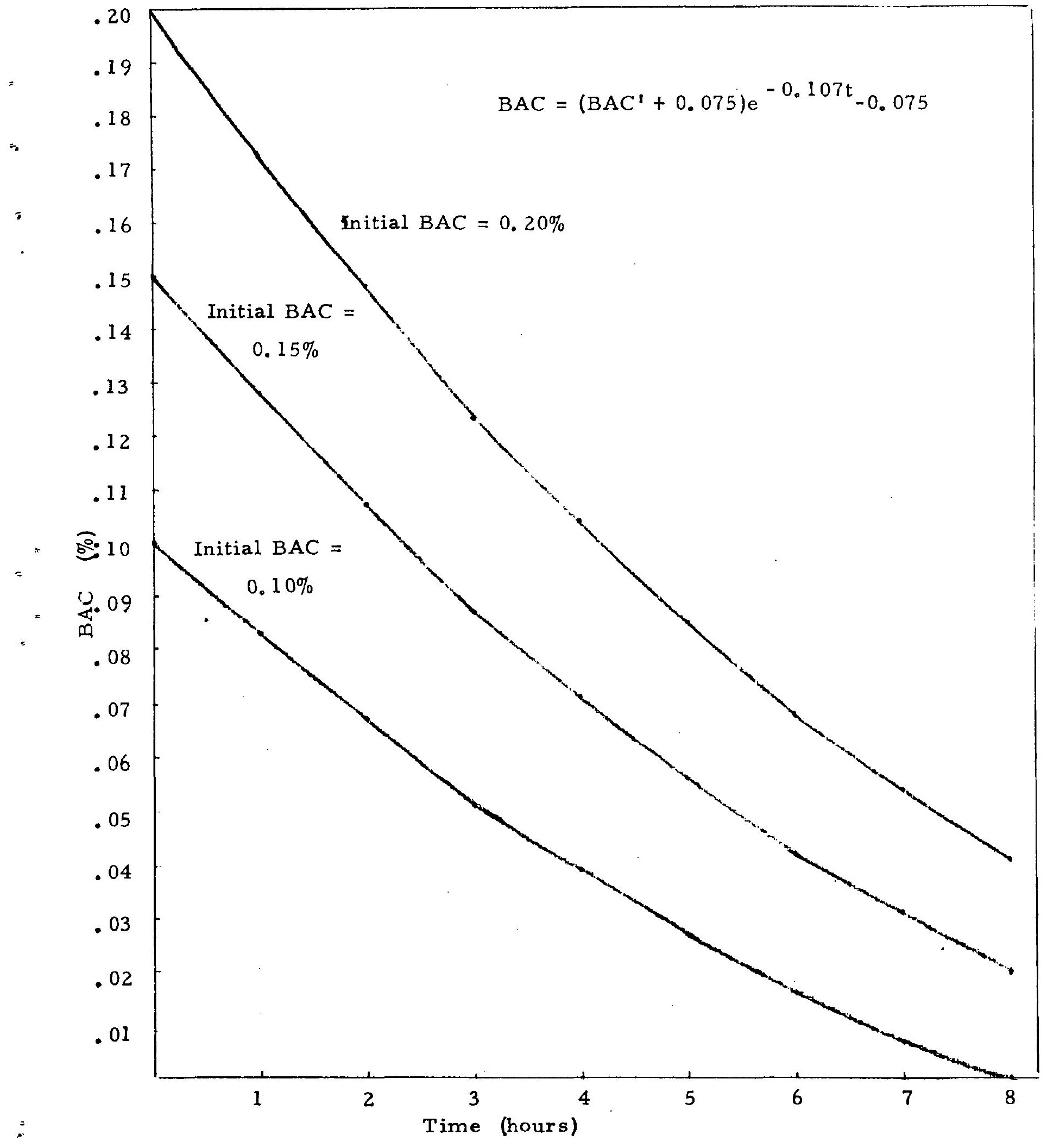

Figure A-3. Theoretical Relationship Between BAC and Time (after complete absorption)

$$
\text { A }-9
$$


and Staub* found that doses of 0.9 to 1.8 grams of fructose per kilogram of body weight increased elimination rate among their Subjects some $48-68 \%$ above the expected value of $0.015 \%$ /hour. Other studies report increases ranging from 0 to $80 \%$ with similar doses, with wide individual differences noted.

All alcohol administered to the Subjects participating in this program was mixed with fruit juices, most of which have appreciable concentration of fructose. Although the exact amounts of juices administered were neither recorded nor measured, it is estimated that a $120 \mathrm{lb}$. Subject ingesting four standard doses of alcohol with orange juice ** would have consumed $1.12 \mathrm{gm} / \mathrm{kg}$. fructose. A $200 \mathrm{lb}$. Subject under the same circumstances would have consumed $0.52 \mathrm{gm} / \mathrm{kg}$. This difference is attributable to the fact that heavier Subjects required greater amounts of alcohol, thus leaving less room in the drinking cup for juice. Therefore, if fructose were truly a factor in the rates noted in this program, its influence presumably would correlate negatively with body weight. A linear regression equation was estimated from the 61 data points and the mean weights recorded for the Subjects. This equation was found to be:

$$
\text { E.R. }=0.019+0.00004(\mathrm{WGT})
$$

with an $r$ value of 0.16 which is not significant at the 0.10 level and certainly not negative. Elimination rates noted in this program thus do not seem to be correlated with the amounts of fructose ingested.

One other possible factor which cannot be discounted concerns individual differences among the program Subjects. Dr. Lepp Greenberg of the Center of Alcohol Studies, Rutgers University, has stated that fairly high rates of elimination have been noted for "heavy" drinkers in previous studies conducted by himself and his colleagues. In the Dunlap program; elimination rates observed at moderate BACs $(0.12 \%$ - $0.15 \%)$ were produced primarily by Subjects who proved unable to attain and/or tolerate higher concentrations. Rates associated with very high levels were produced by the more experienced (and presumably "heavier") drinkers.

Although none of the program Subjects can be considered "light" drinkers in absolute terms, they may be separated on the basis of their ability to attain BACs of $0.18 \%$ or above. Forty-one (41) of the elimination rate data points

\footnotetext{
"Pletscher, A., Bernstein, A., Staub, H. Experientia, 8, 307-08 (1952).

*** Orange juice has the greatest fructose concentration of all beverages used in the program.

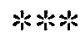

Private communication with the authors. 
were obtained from individuals who achieved these levels at least once in the course of the Program. Their mean elimination rate was $0.029 \%$. The mean for the remaining twenty (20) data points was $0.020 \%$. This difference is significant $(p<.001)$ based upon a t test between the two samples of elimination rates.

Thus, two interpretations of the observation that the elimination rates for the Subjects in this program were substantially higher than those usually found in the literature have been tested. Supporting evidence for both has been found. A significant, positive correlation was found between peak BAC and elimination rate. The data further support an assumption of exponential decay in the concentration of alcohol in the blood--a concept which is generally in accord with other similar metabolic functions. The second interpretation that heavier drinkers have higher elimination rates is also supported by a small, but significant difference in the mean elimination rates for partitioned groups of "heavier" and "lighter" drinkers from among the Subjects of this Program.

In view of the above considerations, no final conclusion concerning the possible dependence of elimination rate on $\mathrm{BAC}$ can be drawn at this time. However, results presented herein certainly suggest the need for a carefully controlled experiment designed specifically to test this phenomenon.

\section{Alcohol Distribution Ratios}

There is virtually unanimous agreement in the literature that an individual's BAC is directly proportional to the amount of ethanol in his body and inversely proportional to his body weight. Widmark* determined that these factors are related according to the following equation:

$$
\mathrm{BAC}=\frac{0.1}{\mathrm{R}} \quad \frac{\mathrm{A}}{\mathrm{W}}
$$

where $A$ is the amount of ethanol (in grams), W the body weight (kilograms), $B A C$ the concentration (\% wt. /vol.), and $\underline{R}$ the distribution ratio of alcohol between the total body and blood. Average values of $\underline{R}$ determined by Widmark were 0.68 for males and 0.55 for females. These figures imply appreciable sex-dependence in the relationship between $\mathrm{BAC}$ and ethanol dosage.

Data obtained in the course of this program provided an opportunity for empirically deriving comparable relationships. Owing to the care taken in assigning the amounts of alcohol during the 99 Subject-days of testing, precise

"Widmark, E: M. P. (1932), as quoted in Alcohol and the Impaired Driver, pg. 19. 
data are available concerning the doses of ethanol ingested per body weight. Similarly, the conduct of multiple breath tests subsequent to ingestion of each dose provided accurate estimates of resulting peak BAC. However, in order to permit comparison with Widmark's formula, these BACs must be adjusted to correct for the alcohol elimination occurring during the period over which the drinks were administered. The careful measurement and recording of these time spans permits these corrections to be made.

Calculation of distribution ratios proceeded as follows: the total amount of ethanol ingested by each Subject on each testing session, coupled with the highest GC breath measurements subsequently recorded, provided 78 data pairs.* The amounts and measurements corresponding to the first two drinks of each session provided an additional 75 pairs. $* *$ For each of the 153 data pairs, the corresponding time span commenced with the start of the first drink and ended with completion of the last in the series (i. e., final or second drink of the test session).

Two distinct techniques for correcting for alcohol elimination over the time interval were investigated. The first assumed a constant rate of elimination of $0.015 \%$ per hour. The products of this rate and the time intervals were added to the peak BACs to determine theoretical maximum concentrations corresponding to the ethanol doses. The second technique attempted to account for the elimination rate's possible dependence on BAC discussed in Section 1 of this Appendix. An approximate rate was computed for each data pair by employing Equation $A-2$ and by assuming that the average concentration during the time interval was one-half the corresponding peak BAC. The products of these rates and time intervals, when added to the recorded BACs, provided a second set of theoretical maximum concentrations.

Linear regression equations then were computed between the amounts of ethanol doses $(\mathrm{gm} / \mathrm{kg})$, and these two sets of theoretical concentration, with the following results:

* Data obtained during test sessions in which the GC was inoperative were not employed for this analysis.

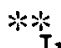

In three instances, the first two drinks of the session accounted for all the ingested alcohol for which subsequent breath tests results were a vailable. 
- Case I - (assuming constant elimination rate)

(Eqs. A-6-I)

$\begin{array}{lll}\text { All subjects } & \mathrm{BAC}=0.136 \frac{\mathrm{A}}{\mathrm{W}}+0.005, \quad \mathbf{r}=0.96 \\ \text { Males } & \mathrm{BAC}=0.133 \frac{\mathrm{A}}{\mathrm{W}}+0.006, \quad \mathrm{r}=0.95 \\ \text { Females } & \mathrm{BAC}=0.141 \frac{\mathrm{A}}{\mathrm{W}}+0.001, \quad \mathrm{r}=0.97\end{array}$

- Case II - (assuming elimination rate proportional to BAC) (Eqs. A-6-II)
All Subjects
$B A C=0.150 \frac{A}{W}-0.010, \quad r=0.96$
Males
$\mathrm{BAC}=0.147 \frac{\mathrm{A}}{\mathrm{W}}-0.009, \quad \mathrm{r}=0.95$
Females
$\mathrm{BAC}=0.155 \frac{\mathrm{A}}{\mathrm{W}}-0.015, \quad \mathrm{r}=0.97$

The correlation coefficients $(r)$ are significant at the .001 level in all cases. Variation in ethanol dosage thus clearly accounts for the vast majority of the variation $(>90 \%)$ in both sets of theoretical peak BACs; this suggests that the basic form of Widmark's formula is the appropriate means of expressing the relationship between these variables.

Next, by computing the quotient of the theoretical BACs and the corresponding ethanol doses, a Distribution Ratio $(\underline{R})$ was derived for each data pair and correction technique. These values are summarized below.

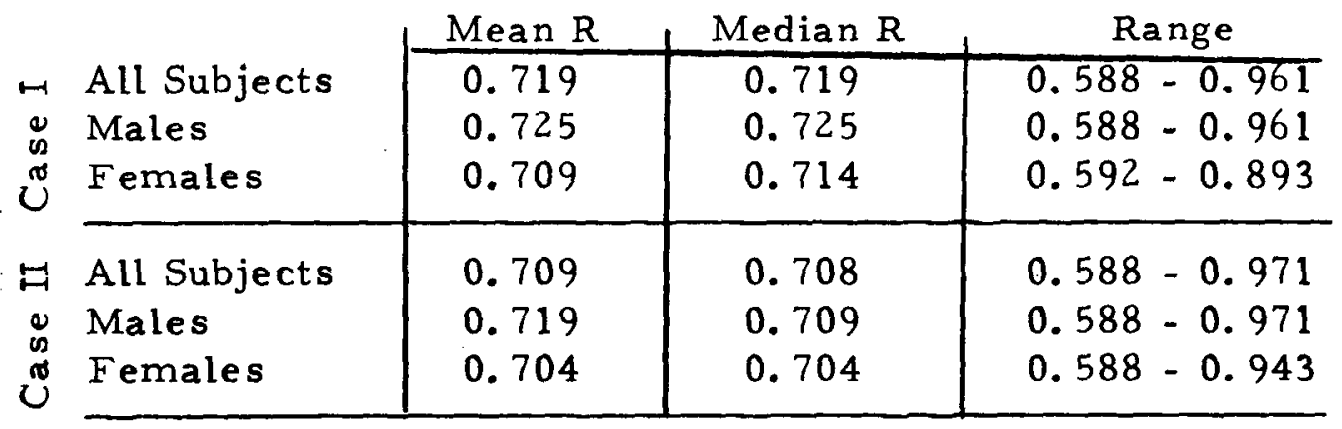

It can readily be seen that the two correction techniques produced very similar results. This is easily understandable, since the calculated concentration-dependent elimination rates (Case II) averaged $0.0157 \%$ per hour, which is not appreciably different from the $0.015 \%$ per hour rate assumed in Case I. Hence the two sets of theoretical maximum BACs were in fairly close agreement. 
It is also evident that the mean values of $\mathrm{R}$ computed for male Subjects conform fairly well to Widmark's finding. While the approximate value of 0.72 derived from the program data is some $6 \%$ higher than the stated figure of 0.68 , this difference might be attributable to such factors as:

- The difficulty in recording the highest BAC actually achieved. If the breath test was conducted either prior to or after attainment of the true peak, the estimate it provides would err on the low size;

- The probability that some Subjects--despite instructions to the contrary--had eaten shortly before the test session, thus reducing their rates of alcohol absorption;

- The fact that highly concentrated doses of ethanol--such as those issued during the program--also are known to contribute to lower than normal rates of absorption.

Each of these factors would tend to lower the peak BAC attained and/or estimated, thus raising the value of $\mathrm{R}$.

The most striking point to be noted in these results is the considerable difference between the calculated $\underline{R}$ values and Widmark's findings as applied to females. The derived value of approximately 0.70 is some $24 \%$ higher than the expected result of 0.55 , a difference not felt to be fully attributable to the possible sources of error listed above.

In searching for possible explanations of this anomaly, it is of value to reflect on the physical interpretation of the distribution ratio. This constant is intended to help quantify the amount of blood corresponding to a given body weight. For most practical applications (i. e., controlled drinking experiments, such as this program) it is generally assumed (tacitly) that, if one Subject weighs twice as much as another, he will have twice the amount of blood in his body, and will therefore require twice the alcohol dosage to achieve the same BAC. However, if a substantial portion of the weight differential is accounted for by significantly different bone structure, allotment of adipose tissue, or other bodily components poor in blood, the assumed linear relationship may not be valid. Specifically, if two individuals are of equal weight, but one has a greater amount of adipose tissue, his volume of blood will be lower and he will achieve a higher BAC per unit dosage of ethanol; that is, he will exhibit a lower $\mathrm{R}$ value. If this hypothesis is correct, $\underline{R}$ will correlate negatively with obesity. 
To test this hypothesis, a simple, quantitative measure of obesity was defined as the quotient of the body weight (in lbs.) and height (in inches). This measure was calculated for each of the 37 program Subjects. The mean value for the 20 males was $2.72 \mathrm{lbs}$. /inch, with a range of 2.06 to 3. 50; the mean for the 17 females was 1.99, with a range of 1.66 to 2.45. To account for the presumed sex-dependence of this measure, values were normalized by dividing by the appropriate mean value cited for male (2. 58) and female (2.09) drivers *. This procedure provided a sex-independent measure of the relative obesity of each Subject based upon current populational norms. Linear regression equations were then estimated between this variable (designated $O B$ ) and the previously calculated $\underline{R}$ values, with the following results:

Case I

All Subjects:

Males:

Females:

Case II

All Subjects:

Males:

Females:
(Eqs. $A-7-I$ )

$$
\begin{aligned}
& \underline{R}=0.93-0.20(O B), r=-0.38 \\
& \underline{R}=1.01-0.26(O B), r=-0.49 \\
& \underline{R}=1.02-0.33(O B), r=-0.48
\end{aligned}
$$

$$
\text { (Eqs. A-7-II) }
$$

$\underline{\mathrm{R}}=0.94-0.22(\mathrm{OB}), \mathrm{r}=-0.36$

$\underline{\mathrm{R}}=1.00-0.26(\mathrm{OB}), \mathrm{r}=-0.44$

$\underline{R}=1.07-0.38(O B), r=-0.49$

All correlation coefficients were found to be significant at the .001 level, thus clearly supporting the hypothesis.

The preceding analysis provides the basis for a possible explanation of the lack of appreciable difference between the male and female distribution ratios empirically derived from the program results. The regression equations clearly indicate that, for a given level of relative obesity, the female $\underline{R}$ will be less than the male. However, this difference is appreciably affected by variations in the respective values of $O B$ for the two sexes. Specifically, the difference will increase if the female is relatively more obese than the male, and will diminish if the opposite is true. The female Subjects in this

\footnotetext{
Stoudt, H. W. and McFarland, R. A. Anthropometric Characteristics of Automobile Drivers, SAE Paper No. 700358; pg. 63.
} 
program had a mean $O B$ of 0.95 , the males 1.05 . If the se figures had been exactly reversed, the regression equations indicate a difference of roughly .08 would have been exhibited between their respective ㅌv values.

This difference is not sufficient to match Widmark's reported sex difference, but a different populational norm where females were more obese (relatively) than is presently the case could easily provide $O B$ values and $\underline{R} s$ which align with the earlier findings. It is suggested that the $R$ differential between males and females is at least partially a function of populational norms* of obesity for the two sexes.

In summary, the results of this program support the dependence of BAC upon ethanol dose and body weight expressed in Widmark:'s equation, and also support the asserted sex-dependence of the distribution ratio. However, this latter dependence appears sensitive to variations in level of obesity, and should therefore be susceptible to wide individual differences.

"Widmark's data represent Europeans in the late $1920 \mathrm{~s}$ or early 1930s. The sample here is from a northeastern suburban U.S. area in 1972. 
The requirement to select Subjects with previous exposure to elevated BAC indicated need for a technique for quantifying a candidate's drinking pattern. To satisfy this need, the project staff developed a pre-selection screening instrument, which subsequently was applied to assess the suitability of all candidate Subjects. This appendix discusses the origins and content of this instrument, documents the results obtained from its employment, and discusses its merits for future applications.

\section{The Screening Instrument}

The instrument, presented in Exhibit B-I, consists of a structured set of queries subdivided into three parts. Part $A$ is intended to elicit essential background information from the candidate, to formally establish that he:

- Has drinking experience

- Has driving experience

- Possesses reasonable physical and psychomotor faculties

- Will not be endangered by the ingestion of alcohol

- Can satisfy the scheduling requirements of the program

Part $A$ also serves to set the stage for the interview. Its "neutral" questions permit gradual introduction of the more personal topics discussed in Parts $B$ and $C$, thus reducing the candidate's anxiety and enhancing the likelihood of obtaining factual, accurate responses.

Part B contains seven questions designed to explore the amount, frequency, and circumstances of the candidate's typical drinking experience. This part of the instrument was derived primarily from a questionnaire developed by the Vermont Alcohol Safety Action Project for use in its roadside interview program. A set of response class intervals is defined for each of these seven questions, and a numeric weight is assigned to each possible response. These weights reflect the Dunlap staff's subjective assessment of the importance of each response as an indicator of Subject suitability. 
Part $C$ consists of nine questions which delve into gerierally recognized indications of "heavy" drinking. These nine are a (slightly modified) subset of the Michigan Alcoholism Screening Test (MAST)*. The numeric weights assigned to the responses to these questions were also derived from MAST.

2. Pre-Test of the Instrument

At the suggestion of the Contract Technical Manager, a pre-test of the screening instrument was conducted. The objective of the test was to determine what, if any, relationship exists between ability to achieve a BAC of roughly $0.15 \%$ and score on Parts $B$ and $C$ of the instrument. A total of fourteen Subjects participated in this test which was conducted in the manner set forth below.

Each Subject, after completing the medical examination conducted by the attending physician, submitted to an initial Breathalyzer test to verify that his blood alcohol concentration was negligible (i.e., 0.00\%). At that point, each was privately interviewed and the screening instrument scores recorded. Based upon his weight, determination was made of the amount of 190 Proof Grain Alcohol required to achieve a BAC of $0.15 \%$ within three hours. Each Subject was required to imbibe two (2) drinks, each containing 1/4 of the calculated amount of alcohol, within the first forty minutes. Smoking was permitted during this drinking interval, but absolutely no eating. At the completion of the forty minutes, a twenty minute waiting period (with no smoking permitted) was observed.

At the end of the waiting period, the Subjects submitted to Breathalyzer tests, the results of which were recorded. When necessary, modifications were made to the amount of alcohol to be contained in the next drink (i. e., if the BAC was higher or lower than the level predicted for that point in time-roughly $0.08 \%)$. All Subjects were given the opportunity to cease drinking at this time; none elected to do so.

The third drink was imbibed during a twenty minute interval, followed by a twenty minute waiting period and a Breathalyzer test. Again, an opportunity to "quit" was offered--only one Subject did so at this point. Appropriate modifications were then made to the alcoholic content of the fourth drink. During the ingestion of that drink, Subjects were permitted to eat potato chips, pretzels, etc. Two Subjects did not finish their fourth drinks.

\footnotetext{
See Selzer, M. L., Vanosdall, F.E., and Chapman, M. "Alcoholism in a Problem Driver Group: A Field Test of the Michigan Alcoholism Screening Test (MAST), " Journal of Safety Research, Vol. 3, No. 4, December 1971, pages $176-181$.
} 
After another twenty minute waiting period, another Breathalyzer test was administered. Two Subject: who proved to be below $0.15 \%$ were given relatively "light" fifth drinks.

At this point Subjects were given light snacks, after which Breathalyzer tests were conducted at roughly 45 minute intervals for the remainder of the session. All Subjects were driven home at the completion of the session.

Table B-I documents the peak BAC and screening instrument scores recorded for each of the fourteen Subjects. Note that, in addition to the three who "quit" before completing their fourth drinks*, four others peaked below $0.15 \%$ \% This apparently was due to underestimation of the necessary alcoholic content of their last drink, and/or underestimation of their elimination rates. In any event, all four asserted that they could easily have imbibed more--thus, they should probably be considered "qualifying" Subjects.

No strong conclusions regarding screening instrument score thresholds could be reached on the basis of this pre-test, since none of the fourteen Subjects ever became ill, overly belligerent, or manifested any other obvious indications of unsuitability. Only one Subject (No. 14) seemed definitely incapable of attaining sufficiently high BAC, with two others (Nos. 11 and 13) indicating marginal capability by virtue of failing to complete the final drink. These three did, however, produce some of the lowest scores on the screening instrument, less than 20 on Part B and zero on Part C in each case. On the other hand, two other Subjects (Nos. 4 and 10) produced similar scores and yet appeared qualified for the ASIS test program.

In the absence of a clearly indicated score threshold, the following general rules for Subject selection were adopted:

1. Individuals scoring at least 25 on Part $B$ would be selected

2. Individuals scoring below 18 on Part $B$ would be rejected

3. Those scoring between 18 and 24 on Part B would be selected if they achieved positive score on Part $C$ of if they exhibited other competent evidence of qualification.

Two common examples of such competent evidence were:

* Subjects 11,13 , and 14
*** Subjects $8,9,12$ and 19 
Table B-I

Results of Pre-Test of Subject Screening Instrument

\begin{tabular}{|c|c|c|c|c|c|c|c|c|c|}
\hline Subject No. & Sex & Age & $\begin{array}{l}\text { Scor } \\
\mathrm{B} \quad 1 \\
\end{array}$ & $\mathrm{C}$ & $\begin{array}{l}\text { No. of } \\
\text { Drinks }\end{array}$ & $\begin{array}{l}\text { Milliliters } \\
\text { Alcohol** }\end{array}$ & $\begin{array}{l}\text { Body } \\
\text { Weight }\end{array}$ & $\begin{array}{l}\text { Peak BAC } \\
\text { Recorded }\end{array}$ & $\begin{array}{c}\text { Session } \\
\text { No. } \\
\end{array}$ \\
\hline 1 & $\mathbf{M}$ & 39 & $24 !$ & 0 & 4 & 170 & 179 & 0.194 & 3 \\
\hline 2 & M & 36 & 32 & 0 & 4 & 169 & 168 & 0.182 & 3 \\
\hline 3 & $\mathrm{M}$ & 25 & 40 & 3 & 4 & 164 & 181 & 0.164 & 1 \\
\hline 4 & $F$ & 50 & 161 & 0 & 4 & 124 & 149 & 0.162 & 2 \\
\hline 5 & $\mathrm{M}$ & 63 & 231 & 1 & 4 & 216 & 255 & 0.161 & 1 \\
\hline 6 & M & 47 & 211 & 1 & 4 & 133 & 172 & 0.159 & 3 \\
\hline 7 & F & 21 & 291 & 1 & 4 & 120 & 161 & 0.158 & 1 \\
\hline 8 & M & 25 & 34 & 1 & 4 & 170 & 189 & 0.149 & 1 \\
\hline 9 & $F$ & 42 & 24 & 1 & 5 & 103 & 124 & 0.149 & 2 \\
\hline 10 & $F$ & 36 & 18 & 0 & 4 & 93 & 122 & 0.146 & 2 \\
\hline 11 & M & 47 & $19 i$ & 0 & $3-1 / 2$ & 160 & 176 & 0.145 & 3 \\
\hline 12 & $F$ & 41 & 171 & 2 & 5 & 136 & 150 & 0.142 & 2 \\
\hline 13 & M & 38 & $14 !$ & 0 & $3-1 / 2$ & 135 & 176 & 0.132 & 3 \\
\hline 14 & $F$ & 44 & 17 & 0 & 3 & 96 & 147 & 0.111 & 2 \\
\hline
\end{tabular}

*190 proof grain alcohol 
- Demonstrated suitability through successful participation in pre-test of the screening instrument;

- Fairly high and frequent usage of distilled spirits, but virtually no use of beer or wine, tending to ameliorate the low score on Part $B$ of the screening instrument

3. Application of the Instrument to ASIS Test Subjects

Screening instrument scores were recorded for each of the thirty-seven individuals selected as ASIS Test Subjects. These scores are presented in Table B-II, together with the peak BACs recorded in each session. Table B-III shows Subjects' weights, amounts of alcohol ingested, and times spent drinking.

4. Discussion of the Instrument's Effectiveness

As measured by the recorded concentrations of blood alcohol, the screening instrument can be considered a highly efficient tool for Subject selection, since peak BAC exceeded $0.18 \%$ on 58 of the 99 Subject-days and failed to reach at least $0.15 \%$ only 15 times. However, it is also clear that it failed to completely eliminate instances of illness. Moreover, it is not felt that a more stringent score threshold for selection would enhance the instrument's efficiency in this regard, since this could have eliminated many qualified Subjects without necessarily rejecting an appreciable proportion of those who became ill.

One modification that might have some merit concerns Question \#11, Part $B$, which indicates the candidate's previous incidence of alcohol-induced illness. As the instrument is presently constructed, response to this question adds to the Part $B$ score, an approach reflecting the view that frequent ingestion of alcohol to the point of vomiting indicates a pattern of "heavy" drinking. For the purposes of Subject selection, it might be preferable to assign negative weights to these responses. The potential merits of this approach are indicated by the facts that six of the ten Subjects who became ill stated that they had done so within the previous 12 months, whereas only nine of the remaining twenty-seven Subjects had such experiences.

In conclusion, the screening instrument presented above--with, perhaps, some minor modifications - - is a reasonably effective means of identifying individuals who can attain high levels of blood alcohol concentration. It thus appears valuable for controlled drinking experiment applications. 
Table B-II

ASIS Test Subject Screening Scores and Peak BACs

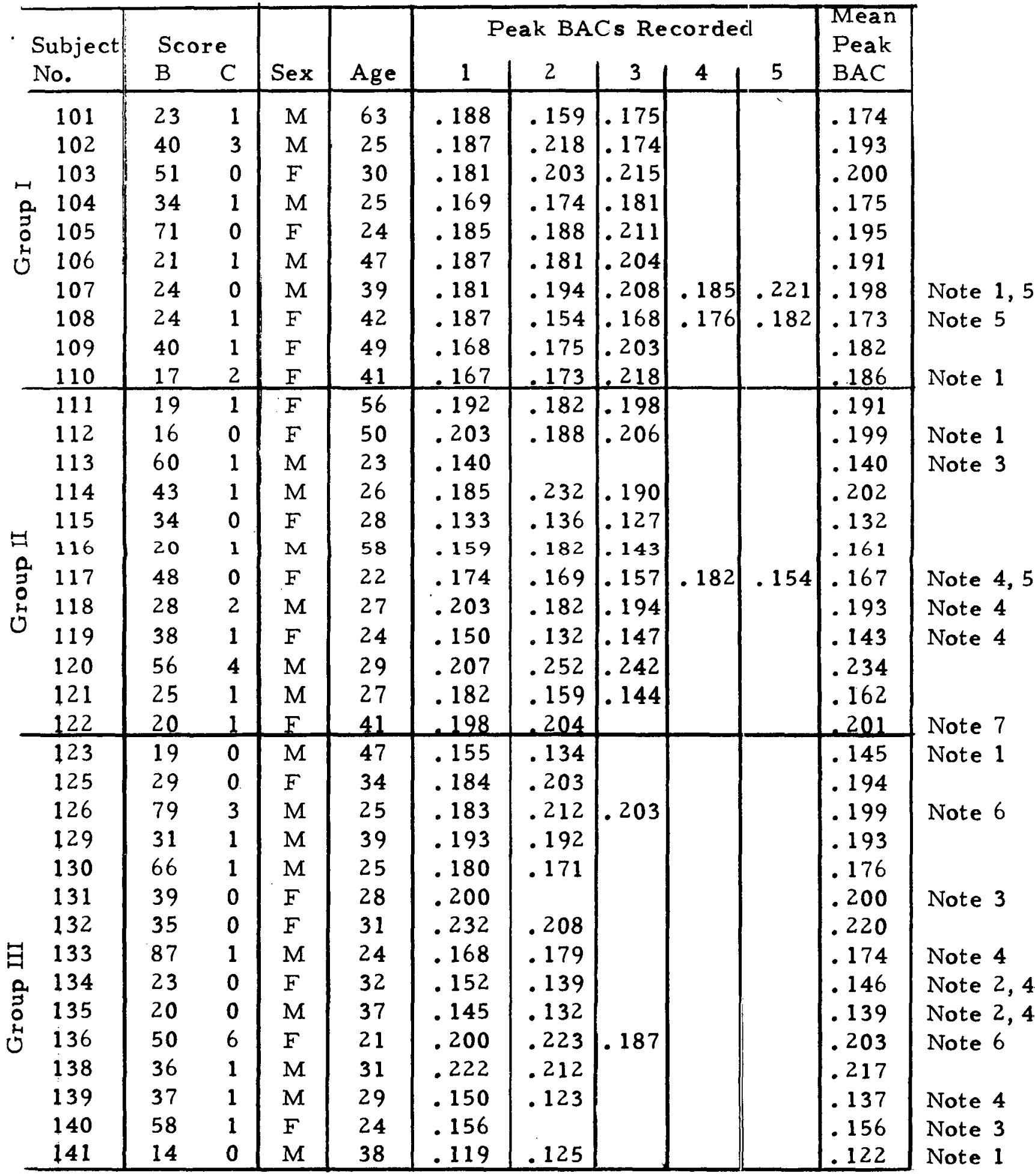


Note 1. Subjects $107,110,112,123$, and 141 were admitted to the program in spite of their relatively low screening scores because they had demonstrated some degree of suitability through participation in the pre-test of the screening instrument.

Note 2. Subjects 134 and 135 likewise were admitted to the program since their low scores reflected almost total abstention from beer and wine, but appreciable use of distilled spirits.

Note 3. Subjects 113,131 , and 140 withdrew from the program after becoming ill during their first testing sessions. Subject 113 subsequently admitted overstating the quantity and frequency of his drinking in order to gain acceptance into the program.

Note 4. Subjects 117, 118, 119, 133,134, 135, and 139 also became ill to the point of vomiting during one session each, but were retained in the program.

Note 5. Subjects 107, 108, and 117 each participated in two additional sessions devoted to the special Quickey experiment. They were reassigned ID Nos. 127, 128, and 137 for that experiment. Subject 114--who could not participate in the special experiment due to poor health--had been reassigned number 124 .

Note 6. Subjects 126 and 136 each participated in one additional session to make up for the withdrawal of Subjects 131 and 140.

Note 7. Subject 122 did not participate in a third session due to a conflict in schedule. 
Table $B-3$

Alcohol Doses

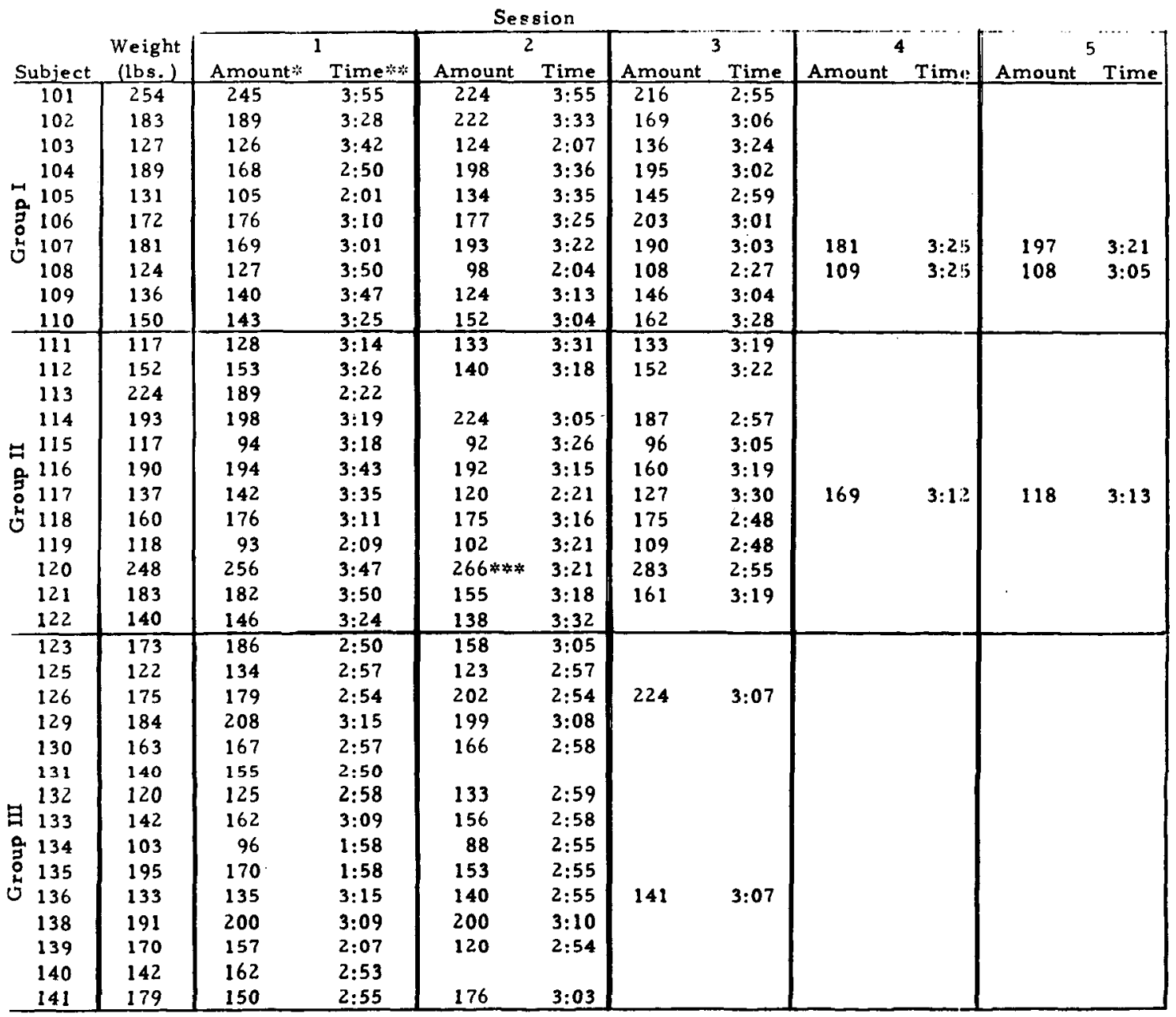

$\begin{array}{ll}2 & \\ 2 & \\ 0 & -1 \\ 0 & 0 \\ 0 & 0 \\ 0 & 0 \\ 0 & 0 \\ 0 & 0 \\ 0 & 1 \\ 0 & 1 \\ 0 & w\end{array}$

$*$ Amount $=$ Milliters of 190 Proof Alcohol

*Time =Duration from start of 1 st drink to completion of last.

*** On his second drinking session and after ingestion of his fourth drink, Subject 120 had to be forcibly restrained from ingesting additional alcohol. It was later concluded that he had consumed one or both of two drinks left incompleted by other $S$ ubjects. The additional amount of alcohol is unknown and not included in this figure. 
EXHIBIT B-I

SUBJECT SCREENING INSTRUMENT

A. Background/Initial Screening

1. Do you ever drink alcoholic beverages? Yes__ No

2a. Have you ever held a driver's license? Yes_ No (If no to either \#1 or \#2a, terminate interview)

2b. When was it first issued? Mo. Year

2c. Is your license currently valid? Yes No If no, why not?

3a. Do you have any nervous or muscular disorders?

Yes (specify)

No

3b. Do you have any problems with your eyesight?

Yes (specify)

No

4. Have you ever been advised by a physician to abstain from, or reduce the amount of, drinking? Yes No

If yes, why?

Name:

Sex: $M$ F

Address : Weight

Telephone:

Date of Birth: Mo. Day Year 


\section{Available for testing:}

Mon. Tues. Wed. Thurs. Fri. Sat. Sun.

\begin{tabular}{|l|l|l|l|l|l|l|l|}
\hline Morning & & & & & & & \\
\hline Afternoon & & & & & & & \\
\hline Evening & & & & & & & \\
\hline
\end{tabular}

B. Drinking Pattern

5. How much distilled spirits (i.e., whiskey, gin, vodka) do you generally drink on any one occasion?

N.A. (doesn't drink whiskey)

One shot $(1--1-1 / 2$ ounces $)$

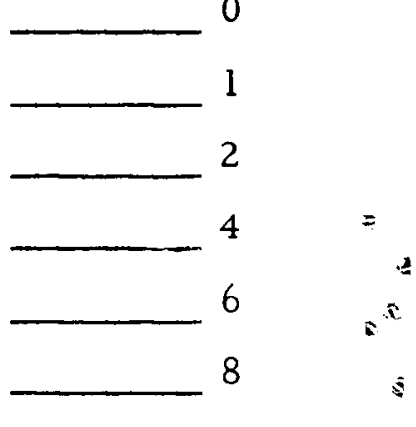

One pint

One pint to one fifth

More than one fifth

6. How much beer do you generally drink on any one occasion?

(N. A. (doesn't drink beer)

One bottle (12 ounces)

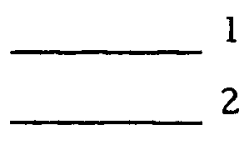

Two-three bottles

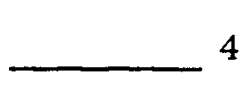

Four-five bottles

One to two six-packs

More than two six-packs

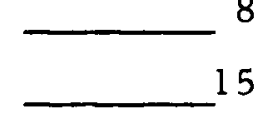

7. How much wine do you generally drink on any one occasion? (N.A. (doesn't drink wine)

One glass (3-4 ounces) 
Two-three glasses 2

Four-five glasses 4

One bottle 10

More than one bottle

8. How often do you drink during:

\begin{tabular}{|l|r|r|r|r|r|}
\cline { 2 - 6 } & Mornings & Lunch & Afternoon & Dinner & Evenings \\
\hline Never & 0 & 0 & 0 & 0 & 0 \\
\hline $\begin{array}{l}\text { Monthly or less } \\
\text { Several times } \\
\text { each month }\end{array}$ & 5 & 1 & 1 & 1 & 1 \\
\hline Wedkly & 15 & 2 & 3 & 2 & 2 \\
\hline $\begin{array}{l}\text { Several times } \\
\text { each week }\end{array}$ & 25 & 5 & 7 & 3 & 3 \\
\hline Daily & 30 & 8 & 10 & 4 & 4 \\
\hline
\end{tabular}

9. Where do you drink most often?

Private home

Bar/restaurant

Other (specify)

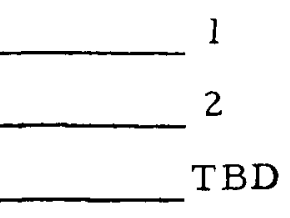

10. When you drink, are you generally

with spouse/family members?

With friends

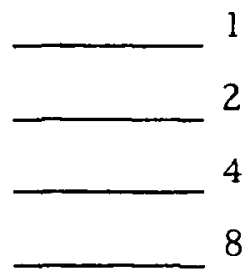

11. How often during the past 12 months have you become physically ill as a result of drinking:

Never 0

Once 
Twice 5

Several or more times

Describe drinking situation at this time(s):

C. Related Indices

12. Have you ever been told that you have alcohol-related kidney disorders, liver trouble, or cirrhosis? Yes (1) No

13. Have you ever had Delirium Tremens, severe shaking, hallucinations? Yes (5) No (0)

14. Have you ever awakened the morning after drinking and found you could not recall a part of the evening? Yes (1) No (0)

15a. Have you ever attended a meeting of Alcoholics Aronymous (AA)? Yes (1) No

15b. If no, has anyone ever recommended that you attend such meetings? Yes (1) No (0)

16. Have you ever seen a clergyman, social worker, doctor, etc. for help with a problem related to your drinking? Yes (1) No

17. Have you ever been in a hospital because of your cirinking? Yes (1) No (0)

18. Have you ever been arrested for "drunk and disorderly" or "public intoxication?" Yes Na. If yes, how many times?

19. Have you ever been arrested for "drunk driving, "driving while intoxicated," or "driving while under the influence of alcoholic beverages?"

Yes No If yes, how many times? $(\mathbf{x} 2)$ 